\title{
A Low-Cost Method of Airspeed Data Acquisition via Image Processing and Experimental Aerodynamic Load Calculation Using Panel Methods
}

\author{
by \\ Shazad Abraham-Doman \\ A thesis submitted to \\ the Faculty of Graduate Studies and Research \\ in partial fulfilment of \\ the requirements for the degree of \\ Master of Applied Science \\ Ottawa-Carleton Institute for \\ Mechanical and Aerospace Engineering
}

Department of Mechanical and Aerospace Engineering

Carleton University

Ottawa, Ontario, Canada

April 2015

Copyright (c)

2015 - Shazad Abraham-Doman 


\section{Abstract}

With large commercial aircraft now containing more than $50 \%$ composite materials by weight, this new trend in aerospace materials has now transferred to the general aviation market segment. These smaller aircraft contain primary structures built with composite materials that exhibit viscoelastic properties, namely an inherent time-dependent load memory not seen in metallic-based materials. A project was undertaken at Carleton University to develop a service-life prediction method for smaller general aviation aircraft comprised of composite primary structures to obtain a greater understanding of their use in this market segment.

Unlike their larger commercial counterparts, most general aviation aircraft do not possess native flight data acquisition systems used to gather necessary in-service information. As such, this thesis presents the development of a low-cost method to acquire experimental flight data and the subsequent aerodynamic load calculations, using common Panel Method theory, to obtain a load history for a composite wing spar. Using readily-available amateur UAV components, the emphasis of the work presented is on the acquisition of airspeed information solely through image processing using cockpit video footage. The preparation of a Diamond DA20-A1 Katana for flight testing purposes is presented along with the required Transport Canada certification process. The results of the developed method show that a load history can be obtained in a very cost-effective manner. A discussion is included on the feasibility of expanding the data acquisition method to develop a flight data recorder for the general aviation market segment. 
I dedicate this thesis to my parents. I found something that interested me. Your encouragement motivated me to pursue it. Your support allowed me to complete it. Thank you. 


\section{Acknowledgments}

I would like to acknowledge the faculty, staff, and students at Carleton University who shared their knowledge, experience, and advice; the project's industry partner Ottawa Aviation Services with their maintenance team and flight instructors; Ashraf Othman, for volunteering his time and expertise without which flight testing would not have taken place; Dr. Robert Langlois and his research team at Carleton University for their continued support; Christopher Goudie for being the catalyst to a number of Eureka moments; and most

of all Dr. Craig Merrett, my advisor, for his understanding, guidance, wisdom, unlimited patience, enthusiasm, encouragement and for pushing me farther than I thought I could go. 


\section{Table of Contents}

$\begin{array}{ll}\text { Abstract } & \text { i }\end{array}$

Acknowledgments

Table of Contents $\quad$ iv

List of Tables $\quad$ viii

List of Figures $\quad$ ix

Nomenclature $\quad$ xii

1 Introduction 1

1.1 Aerospace Composites . . . . . . . . . . . . . . . . . . . . . 1

1.2 Data Acquisition and Flight Test Aircraft . . . . . . . . . . . . 3

1.3 Flight Data Recorders . . . . . . . . . . . . . . . . . . . . 4

1.3.1 Need for Flight Data Recorders in Smaller General Aviation Aircraft 6

1.4 Project Motivation . . . . . . . . . . . . . . . . 7

1.5 Thesis Overview . . . . . . . . . . . . . . . . . . 7

2 The Aircraft $\quad 10$

2.1 Diamond DA20-A1 Katana . . . . . . . . . . . . . . . . . . . 10

3 Potential Flow Theory 13

3.1 Analytical Introduction: Small-Disturbance Flow . . . . . . . . . . . . . 13 
3.1 .1 Assumptions . . . . . . . . . . . . . . . . . . . 13

3.1 .2 Problem Definition . . . . . . . . . . . . . . . . . . 14

3.1.3 Wing Geometry and Boundary Conditions . . . . . . . . . . . . 15

3.2 Analytical Solution . . . . . . . . . . . . . . . . . 18

3.2.1 Source and Doublet Modeling Elements . . . . . . . . . . . . . 20

3.2.2 Symmetric Wing with Nonzero Thickness at Zero Angle of Attack . 23

3.2.3 Zero-Thickness Cambered Wing at Angle of Attack (Lifting Problem) 25

3.2.4 Superposition of the Problems _ . . . . . . . . . . . . . 27

4 Panel Method: Numerical Approach $\quad 28$

4.1 Boundary Conditions . . . . . . . . . . . . . . . . . . . . 30

4.1 .1 Zero Normal Flow Condition . . . . . . . . . . . . . . 30

4.1 .2 Wake Condition . . . . . . . . . . . . . . . . 32

4.2 Modeling Elements . . . . . . . . . . . . . . . . . . 32

4.2.1 Three-Dimensional Constant Strength Source Element . . . . . . . . 33

4.2.2 Three-Dimensional Constant Strength Doublet Element . . . . . . . 36

4.3 Discretization of the Wing into Panels . . . . . . . . . . . . . . . 37

4.4 Influence Coefficients . . . . . . . . . . . . . . . . . . 39

4.5 Creating a Set of Linear Algebraic Equations _ . . . . . . . . . . . . . 40

4.6 Secondary Calculations . . . . . . . . . . . . . . 43

5 Flight Test Preparation $\quad 45$

5.1 Flight Test Plan . . . . . . . . . . . . . . . . . . . 45

5.2 Flight Data Acquisition and Instrumentation . . . . . . . . . . . . 47

$5.2 .1 \quad$ ArduPilot Mega . . . . . . . . . . . . . . . . . . . . 47

5.2 .2 GoPro Video Camera . . . . . . . . . . . . . . . . 48

5.3 Equipment Installation . . . . . . . . . . . . . . . . . . . . . . . 49

5.3.1 Airspeed Pressure Sensor . . . . . . . . . . . . . . . 49

5.4 Equipment and Instrumentation Testing . . . . . . . . . . . . . 50

5.4 .1 Pressure Sensor Test . . . . . . . . . . . . . . 51 
5.4 .2 Low-Temperature Operational Test . . . . . . . . . . . . . . 52

5.4 .3 Pitot-Static System Test . . . . . . . . . . . . . . . . . 52

$5.5 \quad$ Flight Permit . . . . . . . . . . . . . . . . . 53

5.6 Equipment Cost . . . . . . . . . . . . . . . . . . 53

6 Airspeed Determination Through Video Processing 55

6.1 Video Footage and Image Processing . . . . . . . . . . . . . . . . . . 55

6.2 Image Processing and Object Detection in MATLAB . . . . . . . . . . 57

6.2 .1 Binary Image . . . . . . . . . . . . . . . . . . . . 58

6.2 .2 Image Masking and Clutter . . . . . . . . . . . . . 60

6.2.3 Locating the Airspeed Indicator Needle . . . . . . . . . . . . . . . . 61

6.2 .4 Determining Airspeed . . . . . . . . . . . . . 62

6.3 Results and Verification of the Developed Method . . . . . . . . . 63

7 Panel Method Implementation and Aerodynamic Load Determination 66

7.1 Three-dimensional Steady State Panel Method and Verification . . . . . . 66

7.1.1 Coefficient of Pressure Distribution and Coefficient of Lift Calculation 67

7.2 Aerodynamic Lifting Load Determination . . . . . . . . . . . . 69

7.2.1 Discretization of the Wortmann FX 63-137 Airfoil . . . . . . . . . 69

$7.2 .2 \quad$ Aerodynamic Lift Loads . . . . . . . . . . . . . . . . . . . . 71

$\begin{array}{lll}8 & \text { Conclusion } & 73\end{array}$

8.1 Future Work . . . . . . . . . . . . . . . . . . 74

8.1.1 Airspeed Acquisition via Image Processing . . . . . . . . . . . . . . 74

8.1.2 Aerodynamic Load Determination and the Panel Method . . . . . . 77

8.2 Potential Applications . . . . . . . . . . . . . . . . . . . 77

$\begin{array}{ll}\text { List of References } & 79\end{array}$

$\begin{array}{lr}\text { Appendix A } & 83\end{array}$ 
Appendix B Sun Presence Detector 


\section{List of Tables}

2.1 Diamond DA20-A1 Katana Specifications. . . . . . . . . . . . . 10

5.1 Manouevres used in the flight test. . . . . . . . . . . . . . . . 46

5.2 ArduPilot onboard sensors . . . . . . . . . . . . . . . 48

5.3 Approximate cost of equipment needed for aerodynamic load determination. 54 


\section{List of Figures}

1.1 Growth of use of advanced composites in airframe structures. . . . . . . . 2

1.2 Aeroplane accidents in Canada by: (a) operator type (b) activity $\quad \ldots \ldots$. 7

1.3 Service life prediction of an aircraft with a composite material structure. . . 8

2.1 Diamond DA20-A1 Katana. . . . . . . . . . . . . . . . . . . 11

2.2 Three view drawing of the DA20-A1 Katana. . . . . . . . . . . . . . 12

3.1 Finite wing nomenclature. . . . . . . . . . . . . . . . . . . . 14

3.2 Wing thickness, camberline, and upper, lower surface definitions. . . . . . . 16

3.3 Decomposition of the thick cambered wing at an angle of attack into three simpler problems. . . . . . . . . . . . . . . . . 19

3.4 Nomenclature for the potential flow problem. . . . . . . . . . . . 20

3.5 Velocity potential on bounding surface $S_{B} \ldots \ldots \ldots \ldots$

3.6 (a) Wing thickness function (b) Modeling the thickness problem by a source

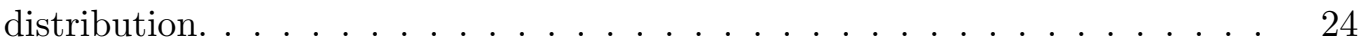

3.7 (a) Wing camber function $\eta_{c}$ (b) Lifting-surface model of a three-dimensional

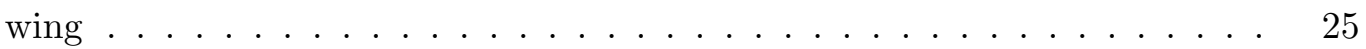

4.1 Flowchart of the numerical approach . . . . . . . . . . . . 29

4.2 Potential flow over a closed body . . . . . . . . . . . . . . . . 31

4.3 Kutta condition using a surface doublet distribution. . . . . . . . . . . 32

4.4 The influence that panel $k$ has on collocation point $P \ldots \ldots \ldots$

4.5 Constant strength source element. . . . . . . . . . . . . . 34

4.6 Constant strength doublet element. . . . . . . . . . . . . . . . . 36

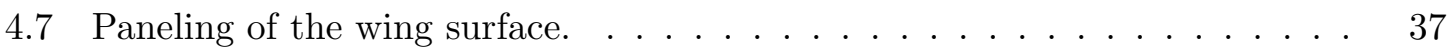


4.8 Method of two-dimensional paneling using the "full-cosine" method. . . . . 38

4.9 Three-dimensional paneling of the wing surface. . . . . . . . . . . . 39

4.10 Wake doublet strength definition using trailing upper and lower panel doublet strengths. . . . . . . . . . . . . . . . . . . . 41

4.11 Local coordinate axes used to determine surface velocities. . . . . . . . . . . 43

5.1 ArduPilot Mega (APM) used to centralize flight data acquisition. . . . . . . 47

5.2 GoPro video camera. . . . . . . . . . . . . . . . . . . . . . . 49

5.3 Installation of flight test equipment in the rear baggage compartment. . . . 50

5.4 Diamond Katana airspeed indicator. . . . . . . . . . . . . . 50

5.5 Differential pressure sensor installed for airspeed information. . . . . . . 51

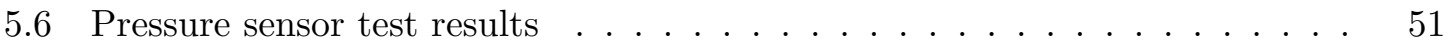

6.1 GoPro camera view of the Katana's instrument panel with the airspeed indicator outlined in red. . . . . . . . . . . . . 56

6.2 Processing images (a) cropped image (b) binary image (c) masked binary image showing only the ASI needle. . . . . . . . . . . . 57

6.3 Luminance variation in cockpit footage. . . . . . . . . . . . . . . . 59

6.4 Image processing method for airspeed determination. . . . . . . . . . . 60

6.5 Depiction of the masked sections of the ASI to isolate the needle. . . . . . . 61

6.6 Examples of a binary image with a level value set too: (a) high (b) low. . 62

6.7 Results of MATLAB's edge(A) function to locate pixels and needle location. 62

6.8 Airspeed markers used for interpolation. . . . . . . . . . . . 63

6.9 The KatanaSim motion-based flight training simulator. . . . . . . . . . . . 64

6.10 Airspeed obtained via image processing compared to airspeeds generated by X-Plane. . . . . . . . . . . . . . . . . . . 65

7.1 NACA 0012 discretization using the "full-cosine" method. . . . . . . . . 67

7.23 -D $C_{p}$ distribution of the NACA 0012 airfoil at $\alpha=5^{\circ} \ldots \ldots \ldots 8$

7.3 Lift coefficient convergence via chord-wise panels. . . . . . . . . . . 68

7.4 Experimental and panel method NACA 0012 lift-curve slope. . . . . . . . 69

7.5 Semi-span discretization of the Wortmann FX 63-137 airfoil (not to scale). . 70 
7.6 Determining the number of panels to use based on a $2 \%$ convergence of $C_{l}$. 71

7.7 Aircraft pitch and airspeed data, and resulting calculated lift load. . . . . . 72

8.1 Examples of instances where airspeed data could not be acquired due to: (a) physical obstruction (b) sunlight glare (c) shadows cast on the ASI. . . . . 75

B.1 An example of the sun presence detected by a "Sun Detector" algorithm. . 85 


\section{Nomenclature}

\begin{tabular}{|c|c|c|}
\hline Symbol & Description & Units \\
\hline$B_{k}, C_{k}, C_{l}$ & Influence coefficients & \\
\hline $\mathrm{c}$ & Chord length & $\mathrm{m}$ \\
\hline$C_{l}$ & Lift coefficient & \\
\hline$C_{p}$ & Pressure coefficient & \\
\hline$M a$ & Mach number & \\
\hline $\mathrm{m}$ & Mass & $\mathrm{kg}$ \\
\hline $\mathbf{n}$ & Surface outward unit normal vector & \\
\hline $\mathbf{Q}$ & Fluid velocity vector & $\mathrm{m} / \mathrm{s}$ \\
\hline $\operatorname{Re}$ & Reynolds number & \\
\hline $\mathbf{q}$ & Induced velocity vector & $\mathrm{m} / \mathrm{s}$ \\
\hline$S$ & Surface area & $\mathrm{m}^{2}$ \\
\hline$l, m, n$ & Principal velocity axes in local coordinates & \\
\hline$u, v, w$ & Principal velocity axes & \\
\hline$x, y, z$ & Principal axes & \\
\hline
\end{tabular}


$\rho$

$\sigma$

$\mu$
Wing angle of attack relative to the free-stream fluid velocity radians

Arbitrary wing geometry expression

Velocity potential

Fluid density

$\mathrm{kg} / \mathrm{m}^{3}$

Source modeling element

Doublet modeling element 


\section{Chapter 1}

\section{Introduction}

General aviation encompasses all aircraft other than those used for military and scheduled flights. It is an important segment of the aerospace industry in Canada and includes operations associated with flight training and private-pilot flights. These flights are typically carried out with single-engined utility aircraft which can be routinely seen around smaller airports and the over 100 flying clubs and flight training centres across the country $[1,2]$.

The aerospace industry has benefited immensely with improvements in aviation-related technology designed to increase aircraft performance and safety. Unfortunately, these gains in technology are mainly directed towards and adopted first by larger commercial aircraft and their operators. This is primarily due to the high costs associated with certification and installation of these systems/methods which cannot be easily recouped by the smaller private pilots or flight training centres. Two of these improvements are the use of composite materials and the introduction of flight data recorders (FDRs). The historical use of these two pieces of technology in aviation and their inclusion/exclusion in the general aviation market segment are discussed in the following sections along with the motivation for the research project and an overview of this thesis.

\section{$1.1 \quad$ Aerospace Composites}

Composite materials were first introduced for use in aircraft during the mid-1970s in limited applications on military fighters. In 1977, Boeing developed a graphite-epoxy horizontal 
stabilizer as part of the NASA-ACEE (Aircraft Energy Efficiency) Advanced Composite Structures Program [3]. This program challenged large-transport manufacturers to use graphite material in redesigning existing aircraft components with the goal of developing the necessary data and technology to achieve production commitments to advanced composites [3]. This would mark the first time a composite primary structure was introduced in a commercial aircraft. The main advantage to composite aerospace structures at this time was the increased performance over metallic-based structures. This led to composites being used in secondary structures throughout the 1980s to mitigate risk and cost [4]. Today, composites are now used extensively in primary structures of commercial aircraft due to weight reduction gains which translates to lower operating costs. Airbus' A350 and Boeing's 787 contain more than $50 \%$ composite material by weight [4]. This trend of increased composite use in aerospace structures, shown in Figure 1.1, is expected to continue and has already transfered to the general aviation market segment.

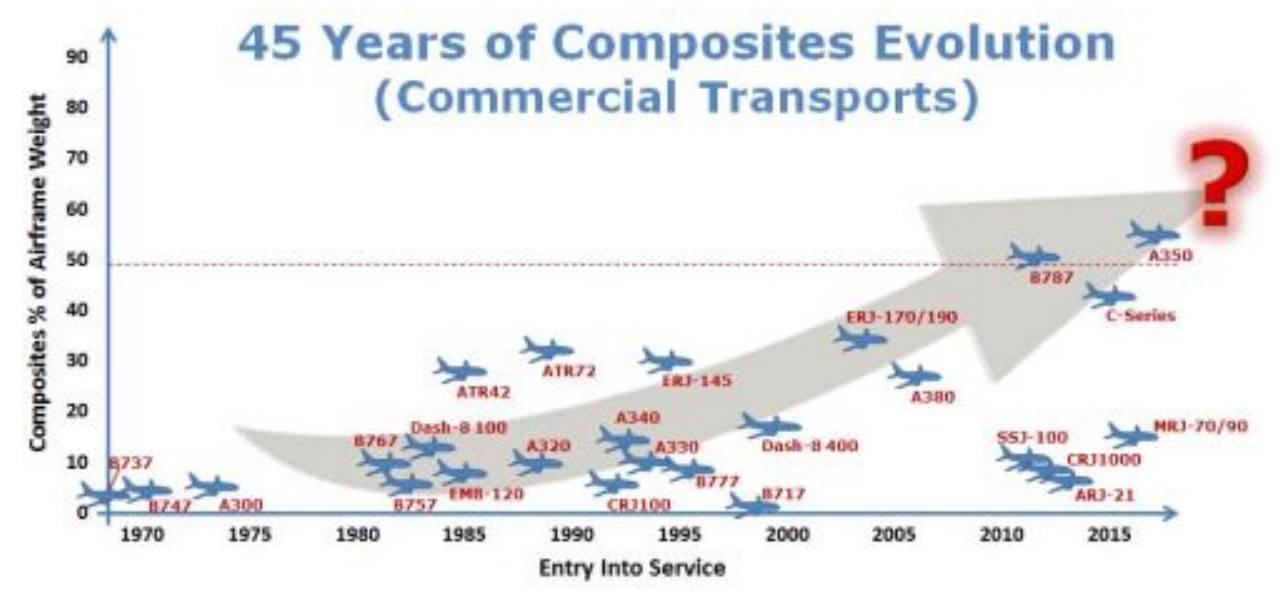

Figure 1.1: Growth of use of advanced composites in airframe structures. [5]

The extensive knowledge of metallic-based structures used in aerospace applications gathered over many decades is not directly transferable to non-metallic structures. This poses a problem due to the extensive list of possible composite materials used and their widely varying responses and failure mechanisms. One of these responses includes the tendency to exhibit viscoelastic properties, namely a load memory. A vast amount of research has been undertaken on composite materials and structures; however, much of this 
has been done by the major aircraft manufacturers and is proprietary [6]. This is further complicated due to the limited in-service information available on composite aerospace structures. For military and large commercial aircraft, which possess native flight data systems to permit gathering of in-service information, analysis techniques can be verified and improved. Unfortunately for the latest adopters of composite materials, general aviation aircraft, native flight data systems are not typical. As such, a method to acquire in-service information for these aircraft that can be used to properly conduct structural analyses is needed.

\subsection{Data Acquisition and Flight Test Aircraft}

Unlike their larger counterparts, the majority of smaller general aviation aircraft are not equipped with native flight data acquisition systems to record basic flight parameters. This is further complicated due to the fact that most of these smaller aircraft still employ mechanical systems to control the aircraft and relay information to the pilot through the various instruments installed within the cockpit. Recording and accessing this information on aircraft without native flight data systems requires the development and installation of such systems, and has generally been limited to flight testing purposes.

Flight testing has always been a crucial component to the development of any aircraft or system and being able to measure an aircraft's performance during flight testing has great implications on the aircraft's safety and marketability [7], in addition to being able to provide in-service information. The costs associated with repeated flight tests is great but using simulations and models decreases these costs while still being able to analyze aircraft flight and system characteristics. This has created a need for accurate flight simulations. With the cost of computer processing resources having decreased over the past three decades, an emphasis on efficient flight modeling of aircraft has taken place, in contrast to repeated flight tests carried out or the historical use of wind tunnel testing and aerodynamic calculations [8]. The generated flight simulations can be used in applications related to aircraft dynamics and control, such as pilot training, mission rehearsal, dynamic 
analysis, and control system design [9]. One such research project found in [8] describes the work to obtain dynamic flight data over a range of flight conditions. This was carried out by using a single flight manouevre which is then used to extrapolate stability and control derivatives for other flight manoeuvres. This work used NASA's Generic Transport Model (GTM) T2 aircraft $[8,10]$, a subscale flight test aircraft specifically designed and instrumented for flight testing purposes.

Other work related to in-service flight information is presented in [11], where an aftermarket digital flight data recorder (DFDR) was installed on a fleet of Beechcraft 1900D commuter aircraft to record operational data. This work was carried out in conjunction with the Federal Aviation Administration (FAA) as part of a continuing effort to understand the issues involved in the aging of aircraft. Very little information is presented in regards to the DFDR used, but the work presented in [11] is an example of the installation and use of a flight data recorder/acquisition system where one was not found natively. The use of such flight data recorders, as it applies to smaller general aviation aircraft, is discussed in the following section.

\subsection{Flight Data Recorders}

Interest in flight data recorders (FDRs) increased following a series of airline crashes in the early 1940s [12]. However, due to World War II, the development of a system and regulations surrounding them did not become available until the late 1950s when the Civil Aviation Board (predecessor to the National Transportation and Safety Board) required that all air carrier aircraft over 12,500 lbs operating above 25,000 feet be equipped with an FDR [12]. The first of these FDRs needed to record altitude, airspeed, heading, and vertical accelerations [12]. This list of parameters was later extended in 1988 to include pitch and roll attitude, thrust for each engine, flap position, flight control input, control surface position, lateral acceleration, pitch trim, and thrust reverser position [12].

Following another series of airline crashes, the list of parameters was progressively increased throughout the 1990s. At this time the FDRs had advanced from using foil 
and magnetic steel wire as recording mediums, to solid-state digital flight data recorders (DFDR) [12]. These systems now required a flight data acquisition unit (FDAU) to convert the analog signals from the sensors to digital signals that were then multiplexed into serial data streams for recording [12]. The introduction of the second generation wide-body aircraft (like the Boeing 767 and Airbus A320) with their digital avionics and fly-by-wire systems allowed a vast amount of information to be available to the FDRs by tapping into the digital data buses that carried digital data between the aircraft systems [6].

The evolution of FDR technology and regulations has, for the most part, encompassed only the transport category of aircraft and has done very little for the small utility aircraft segment. This issue was addressed in 2011 by the International Civil Aviation Organization (ICAO) in their recommendation that small turbine-powered aircraft (below $5700 \mathrm{~kg}$ ) be outfitted with an FDR. As a result of this recommendation the Australian Civil Aviation Safety Authority (CASA) conducted a cost benefit analysis of ICAO's recommendation and found that the costs associated with fitting a recorder in these aircraft would be approximately $\$ 11,000$ per aircraft and that ICAO's "proposal would not generate benefits to justify the costs" [13]. They went on to say

From the perspective of improving aviation safety, the money spent to fit flight data recorders could be used in other ways to improve safety for small aircraft, such as fitting TCAS (Traffic Collision Avoidance System) and or TWAS (Terrain Awareness and Warning System) that would directly improve safety by working to prevent accidents.

Although this analysis was conducted for turbine-powered aircraft, it is clear that cost is very much a driving issue in outfitting smaller aircraft with FDRs.

In addition to FDRs, the installation of image recorders within cockpits has been debated. Image recorders are essentially cameras used to visually document flights. Following the tragic events of Swissair Flight 111 in 1988, the Transportation Safety Board of Canada in a 2003 recommendation [14] stated that

Image recording in the cockpit will substantially benefit safety investigations. 
It will provide investigators with a reliable and objective means of expeditiously determining what happened. This will assist safety investigators in focusing on why events took the course they did, what risks exist in the system, and how best to eliminate those risks in the future.

However, an opposing stance was taken by the FAA as they believed there was "no compelling evidence to require installation of cockpit image-recording systems" [14]. With changes to international aviation regulations seldomly occurring without FAA approval, it seems as though the installation of image recorders in transport aircraft will not occur in the near future. But the use of image recorders in smaller aircraft, as an economical alternative to the installation of FDRs, has been recommended by ICAO [15].

\subsubsection{Need for Flight Data Recorders in Smaller General Aviation Air- craft}

Here in Canada, accidents involving small aircraft continue to outnumber those of their larger counterparts. Figure 1.2 (a) and (b) show accident occurrences in Canada over the past 10 years by operator type and by activity respectively. This information shows a higher incidence rate with private pilots, flight training, as well as air taxi services; all of which are part of the general aviation segment.

The inclusion of a low-cost FDR onboard smaller general aviation aircraft would allow, as recommended by the TSB in [14], a means of determining the causes of the over 200 incidents that occur each year. The information acquired from these aircraft could also be used in a multitude of applications other than accident reconstruction. One such application is the acquisition of flight data for the previously-introduced need for structural analyses.

Cost still remains a very large hurdle in outfitting small general aviation aircraft with FDRs and requires any such system to be cost-effective for the user. The main contributors to these costs are the recorder itself, any associated equipment, labour to install the system, and certification of the system and its installation [13]. 


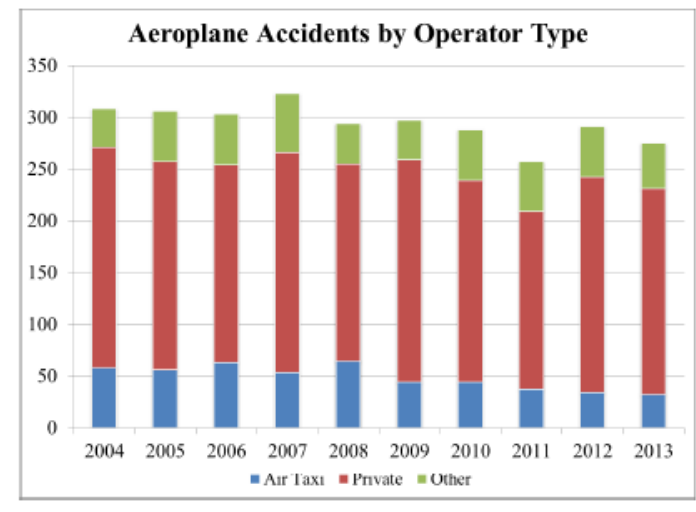

(a)

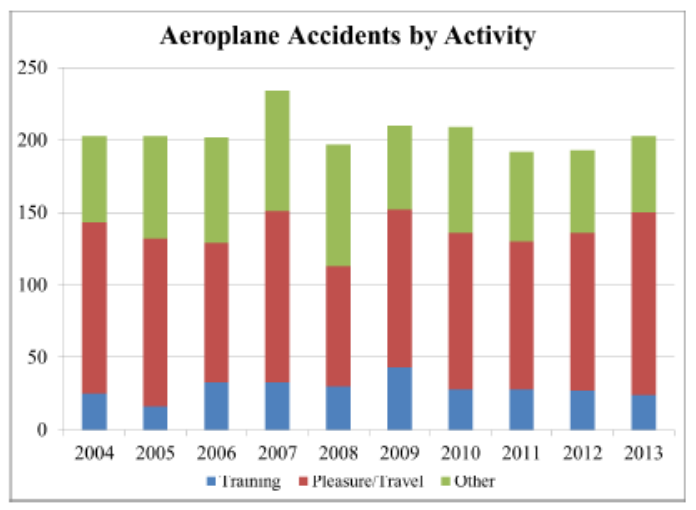

(b)

Figure 1.2: Aeroplane accidents in Canada by: (a) operator type (b) activity (adapted from $[16])$.

\subsection{Project Motivation}

The focus of the research described here is one segment of a larger research program to predict the remaining life of a Diamond DA20-A1 Katana. The Katana is a light utility aircraft with an all composite airframe, widely used for flight training purposes. This aircraft comprises the majority of the fleet at a local flight training centre, Ottawa Aviation Services (OAS), with which the project group has partnered. The manufacturer asserts that the Katana has an unlimited service life [17]; however, comparable aircraft in this market segment have rated service lives of 15,000 to 25,000 flight hours [18]. The aircraft operated by OAS are approaching 10,000 to 12,000 flight hours; therefore, OAS would like to estimate the remaining service life of their aircraft. To do so, in-service information must first be collected that can later be used to determine the aerodynamic loads experienced by the aircraft throughout the remainder of its life.

\subsection{Thesis Overview}

The approach to predicting the service life of a glass-fibre reinforced plastic (GFRP) composite light aircraft has two components, which are shown in Figure 1.3. The first is material and structural response characterization, and the second is modeling of the aircraft loads 


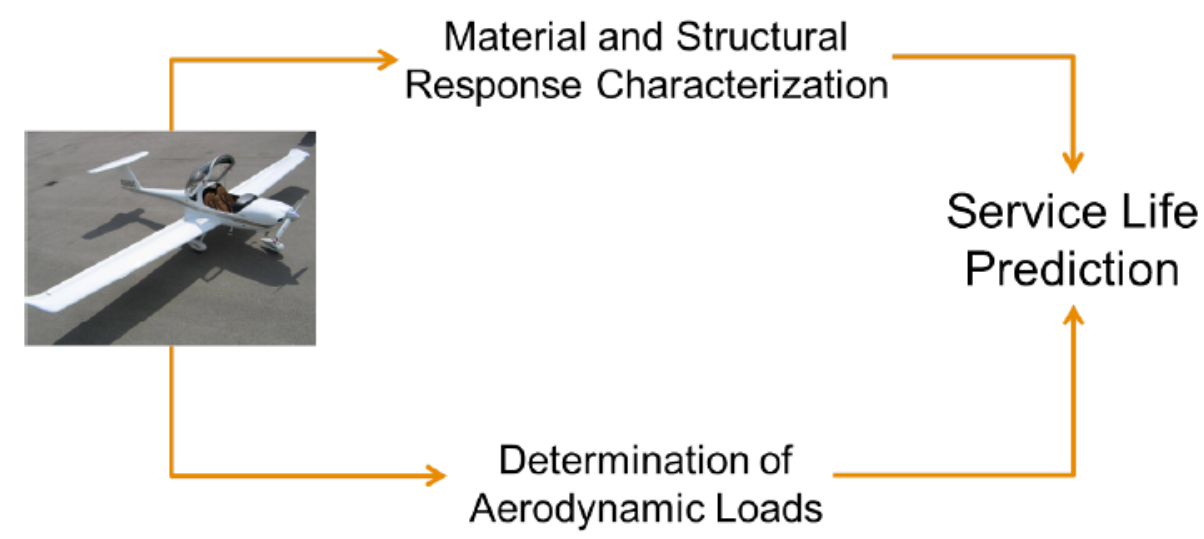

Figure 1.3: Service life prediction of an aircraft with a composite material structure.

during operation. The focus of the reported research is the experimental measurement of the load history on a DA20-A1 Katana wing spar while the material and structural response characterization is being carried out by another Masters student, Christopher Goudie.

Determining aerodynamic loads is of obvious importance to the safety of any aircraft and has been conducted numerous times in industry to calculate these loads using experimental flight data. However, this flight data is commonly acquired using fully-instrumented aircraft as in [8], which is expensive, obtrusive and resource-intensive. This thesis describes the work undertaken to develop a cost-effective flight data system, the work to outfit a general aviation aircraft with the system, airspeed determination through video processing and the use of acquired experimental flight data to determine the aerodynamic lift load on the wing spar.

Chapter 2 provides a brief description of the aircraft used in this work, the Diamond DA20-A1 Katana. Chapter 3 briefly describes potential flow theory and introduces the analytical approach to aerodynamic load analysis. This is followed by an explanation of the numerical approach, the Panel Method, in Chapter 4. Chapter 5 describes the preparatory work for the flight testing used to obtain the experimental flight data. This includes a summary of the flight test plan, a description of the data acquisition system developed and its installation within the aircraft, as well as the required Transport Canada certification 
process. Chapter 6 provides relevant theory related to image processing while simultaneously explaining and illustrating results of the developed method to determine airspeed information through image processing. Chapter 7 uses the results of this image processing method and acquired flight data to present the implementation of the Panel Method theory to obtain aerodynamic lift loads. Chapter 8 finishes with some concluding remarks about the work presented here and its implications. 


\section{Chapter 2}

\section{The Aircraft}

\subsection{Diamond DA20-A1 Katana}

The Diamond DA20 is a low-wing light utility aircraft with an all composite airframe and

a GFRP wing spar. It is widely used for flight training purposes, with the United States Air Force being one of the most notable users of this aircraft [17]. The most basic variant of the DA20, the A1 Katana (Figure 2.1), comprises the majority of the fleet at a local flight training centre, Ottawa Aviation Services (OAS), with which the project group is partnered. The DA20-A1 Katana specifications are outlined in Table 2.1.

Table 2.1: Diamond DA20-A1 Katana Specifications [19]

\begin{tabular}{|c|c|}
\hline Crew & one, pilot \\
\hline Capacity & one passenger \\
\hline Length & $7.17 \mathrm{~m}(23 \mathrm{ft} 6.0 \mathrm{in})$ \\
\hline Wingspan & $10.84 \mathrm{~m}(35 \mathrm{ft} 6.7 \mathrm{in})$ \\
\hline Height & $2.11 \mathrm{~m}(6 \mathrm{ft} 11.0 \mathrm{in})$ \\
\hline Wing area & $11.6 \mathrm{~m}^{2}\left(125 \mathrm{ft}^{2}\right)$ \\
\hline Max. takeoff weight & $730 \mathrm{~kg}^{2}(1609 \mathrm{lbs})$ \\
\hline Powerplant & Rotax 912, variable speed Hoffman propeller, $59.6 \mathrm{~kW}(80 \mathrm{hp})$ \\
\hline
\end{tabular}




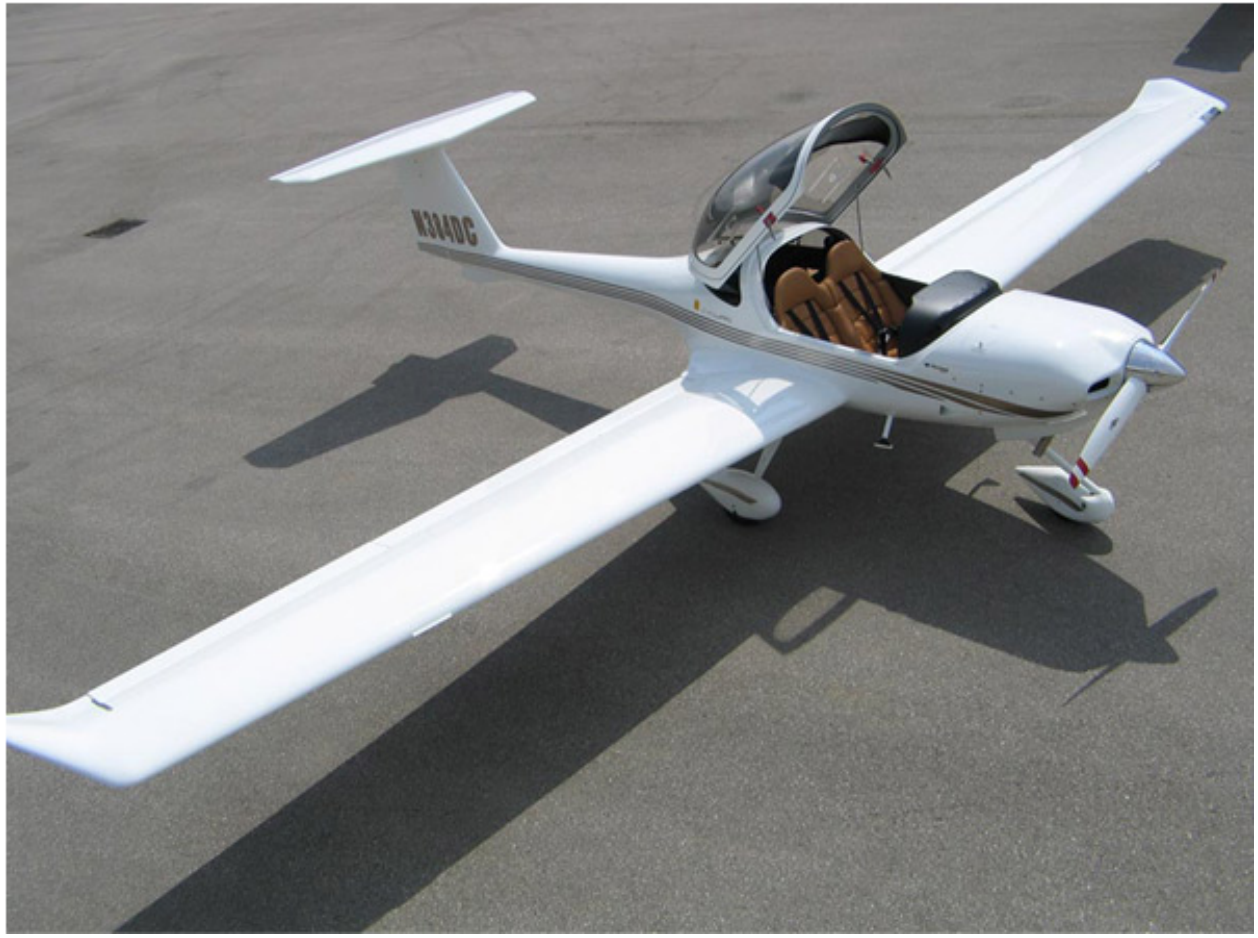

Figure 2.1: Diamond DA20-A1 Katana [17]. 


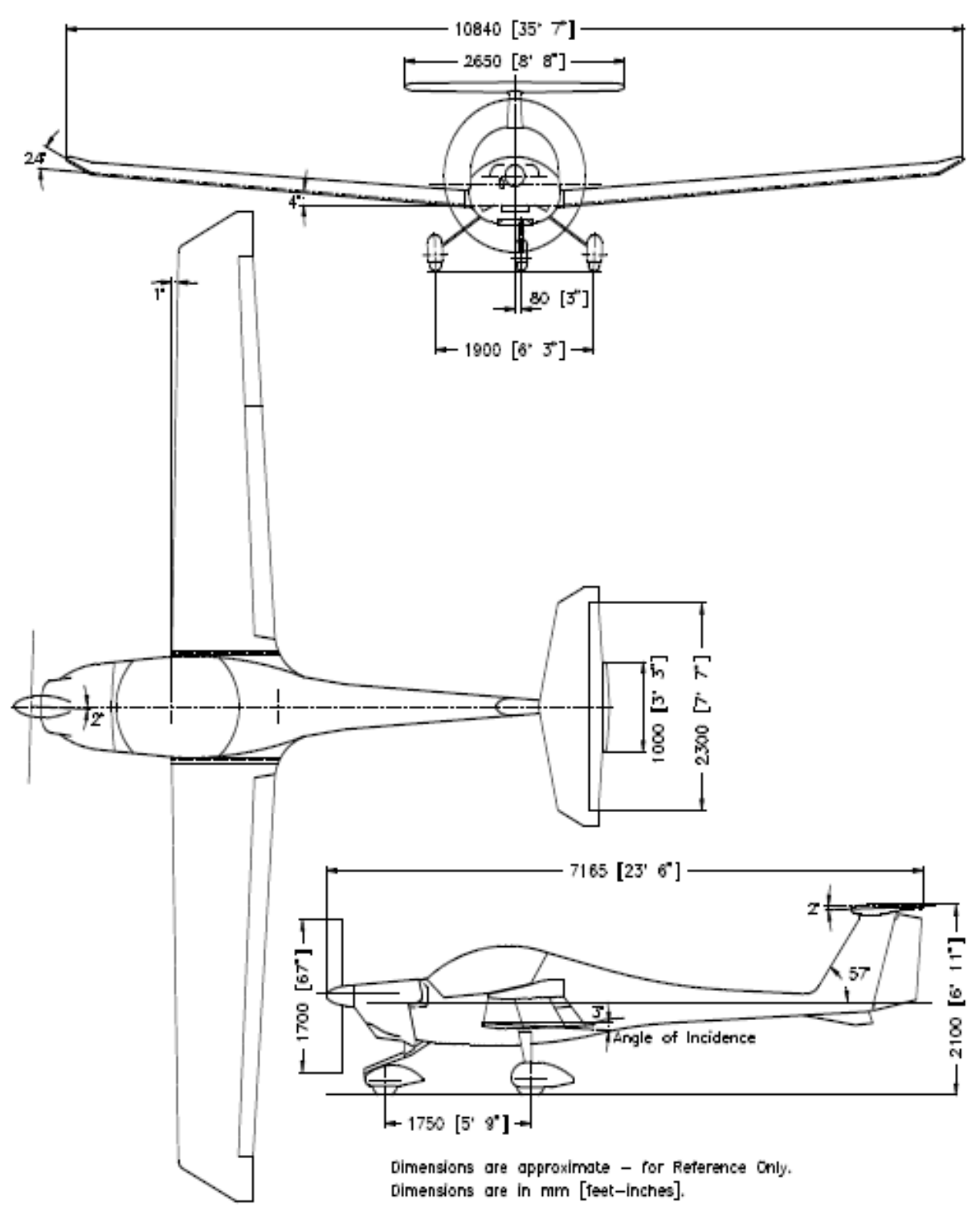

Figure 2.2: Three view drawing of the DA20-A1 Katana. [19] 


\section{Chapter 3}

\section{Potential Flow Theory}

In accomplishing the objectives outlined in Section 1.5, this chapter will show how wing geometry and free-stream velocity can be used to model the flow field around a wing using potential flow theory. This analytical process must first be presented to understand the numerical implementation described later in Chapter 4. The theory outlined in this chapter is adapted from [20] unless otherwise stated. Section 3.1 introduces the potential flow problem using small-disturbance theory. This includes an explanation of the physical problem

and conditions that a solution must meet. Section 3.2 decomposes the problem into three subproblems where modeling elements are introduced and used along with the prescribed conditions to provide a solution.

\subsection{Analytical Introduction: Small-Disturbance Flow}

\subsubsection{Assumptions}

The potential flow problem will be solved using three assumptions:

1. Inviscid fluid (no viscosity).

2. Incompressible fluid.

3. Irrotational flow (no turbulence).

The first assumption prevents any boundary layer analysis from being conducted. The second assumption is valid for low-speed aerodynamic analysis, which is commonly accepted 
as $M a \leq 0.3$ [21] and typical of the slow-moving Diamond Katana described in Chapter 2 . The third assumption is valid when considering the simple flight manoeuvres common to the Diamond Katana and greatly simplifies the numerical analysis that follows.

\subsubsection{Problem Definition}

The solution to the potential flow problem begins by considering the wing of Figure 3.1. A body-fixed coordinate system is used throughout the following sections. The free stream velocity, $\mathbf{Q}_{\infty}$, is described using its principal coordinate axes components: $U_{\infty}, V_{\infty}, W_{\infty}$. For simplicity, no side slip is analyzed,

$$
V_{\infty}=0
$$

an assumption which is discussed further in Chapter 8. The angle of attack, $\alpha$, is taken as the angle between the $x$-axis and the oncoming free-stream velocity:

$$
\alpha=\tan ^{-1} \frac{W_{\infty}}{U_{\infty}}
$$

An outward normal, $\mathbf{n}$, is also used to describe the wing surface and its orientation.

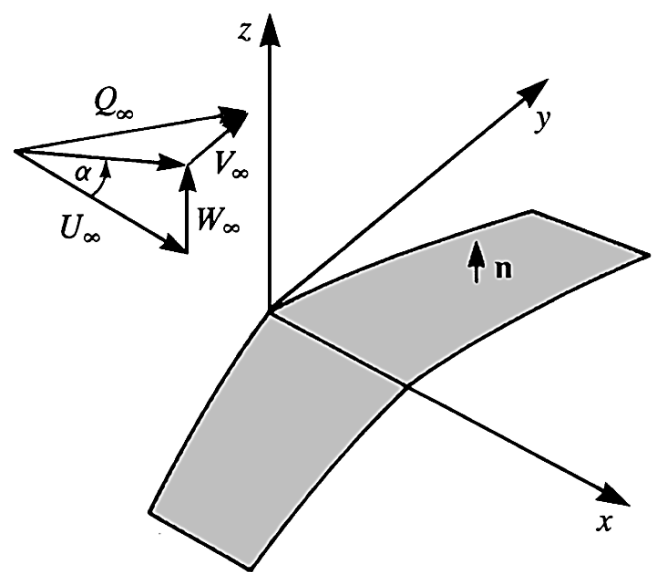

Figure 3.1: Finite wing nomenclature [20].

Provided in [20] is a quite lengthy derivation of the fluid dynamic equations using the three assumptions above. The result of this derivation is the continuity equation

$$
\nabla \cdot \mathbf{q}=0
$$


where $\mathbf{q}$ is an induced-velocity vector that is related to velocity potential in that

$$
\mathbf{q}=\nabla \Phi
$$

yielding

$$
\nabla^{2} \Phi^{*}=0
$$

This is the continuity condition that must be met and used in the solution to follow. Here, $\Phi^{*}$ is the velocity potential and uses a superscript to distinguish terms that will be covered shortly. The first condition applied to the solution is that of a decayed wing-induced disturbance at a distance far from the wing:

$$
\lim _{r \rightarrow \infty} \nabla \Phi^{*}=\mathbf{Q}_{\infty}
$$

The second condition is obtained by considering the physical nature of the problem depicted in Figure 3.1. It is apparent that the normal component of velocity at the wing surface must be zero as this surface is a solid boundary. This condition is expressed mathematically as:

$$
\nabla \Phi^{*} \cdot \mathbf{n}=0
$$

Equations (3.6) and (3.7) must be satisfied by the modeling elements that will ultimately be used to solve the potential flow problem. This solution will generate the induced-velocity vector $\mathbf{q}$ for any point in the flow field along with the associated pressure $p$ using the Bernoulli equation

$$
p_{\infty}+\frac{\rho}{2} Q_{\infty}^{2}=p+\frac{\rho}{2} q^{2}
$$

This pressure information will later be used for secondary calculations, namely the aerodynamic loads induced by this wing in the flow field.

\subsubsection{Wing Geometry and Boundary Conditions}

The driving boundary condition of Equation (3.7) can only be satisfied once the wing geometry is defined. Figure 3.2 shows 4 functions which will be used to do so. Using the 
body-fixed coordinate system shown, the solid surface of the wing is expressed as:

$$
z=\eta(x, y)
$$

The wing of the Katana aircraft is a straight wing with $1^{\circ}$ sweep and $4^{\circ}$ dihedral (see Figure 2.2). The solid surface is then able to be expressed as a function of it chord-wise location $x$ and spanwise-location $y$. Two such functions describing the upper $\eta_{u}$ and lower $\eta_{l}$ wing surfaces are used

$$
\begin{aligned}
& z=\eta_{u}(x, y) \\
& z=\eta_{l}(x, y)
\end{aligned}
$$

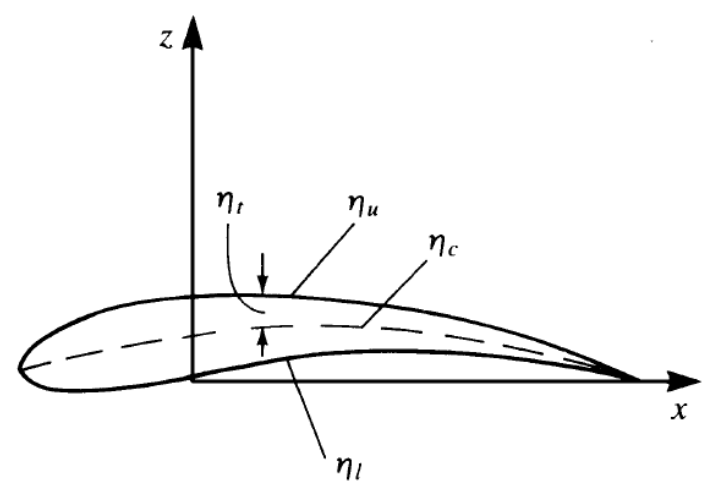

Figure 3.2: Wing thickness, camberline, and upper, lower surface definitions [20].

Additionally, the upper and lower surfaces can be used to define a thickness function $\eta_{t}$ and a camber function $\eta_{c}$

$$
\begin{aligned}
\eta_{t} & =\frac{1}{2}\left(\eta_{u}-\eta_{l}\right) \\
\eta_{c} & =\frac{1}{2}\left(\eta_{u}+\eta_{l}\right)
\end{aligned}
$$

where it follows that

$$
\begin{gathered}
\eta_{u}=\eta_{c}+\eta_{t} \\
\eta_{l}=\eta_{c}-\eta_{t}
\end{gathered}
$$

These various wing geometry expressions are accompanied with a definition of a function $F(x, y, z)$ such that

$$
F(x, y, z) \equiv z-\eta(x, y)=0
$$


solely to allow the outward normal of the upper surface to be expressed using a gradient:

$$
\mathbf{n}=\frac{\nabla F}{|\nabla F|}=\frac{1}{|\nabla F|}\left(-\frac{\partial \eta}{\partial x},-\frac{\partial \eta}{\partial y}, 1\right)
$$

whereas the lower surface outward normal is just the negative of this expression, or $-\mathbf{n}$.

Equation (3.5) is a second-order differential Laplace equation that allows the velocity potential to be expressed as a linear function of position

$$
\Phi=A x+B y+C z \equiv \frac{\partial \Phi}{\partial x} x+\frac{\partial \Phi}{\partial y} y+\frac{\partial \Phi}{\partial z} z \equiv U_{\infty} x+V_{\infty} y+W_{\infty} z
$$

and assuming no side slip (Equation (3.1)):

$$
\Phi_{\infty}=U_{\infty} x+W_{\infty} z
$$

The linearity of this equation allows the velocity potential solution to be separated:

$$
\Phi^{*}=\Phi+\Phi_{\infty}
$$

which explains the earlier use of the superscript in Equation (3.5). At this point Equations (3.19) and (3.20) can be used in the solid boundary condition of Equation (3.7) to yield:

$$
\nabla \Phi^{*} \cdot \mathbf{n}=\nabla^{*} \Phi \cdot \frac{\nabla F}{|\nabla F|}=\left(\frac{\partial \Phi}{\partial x}+U_{\infty}, \frac{\partial \Phi}{\partial y}, \frac{\partial \Phi}{\partial z}+W_{\infty}\right) \frac{1}{|\nabla F|}\left(-\frac{\partial \eta}{\partial x},-\frac{\partial \eta}{\partial y}, 1\right)=0
$$

Here, $\Phi$ is the perturbation potential representing the velocity induced by the motion of the wing in a stationary frame of reference and is the unknown to be solved for. One can therefore deduce that the perturbation potential must be

$$
\nabla^{2} \Phi=0
$$

following the continuity equation (Equation (3.5)).

The first step in solving for the perturbation potential is to rearrange Equation (3.21) and apply the boundary condition on the wing surface:

$$
\frac{\partial \Phi}{\partial z}=\frac{\partial \eta}{\partial x}\left(U_{\infty}+\frac{\partial \Phi}{\partial x}\right)+\frac{\partial \eta}{\partial y}\left(\frac{\partial \Phi}{\partial y}\right)-W_{\infty} \quad(\text { on } \quad z=\eta)
$$


It is at this point that the small-disturbance approximation is assumed:

$$
\frac{|\partial \Phi / \partial x|}{Q_{\infty}}, \frac{|\partial \Phi / \partial y|}{Q_{\infty}}, \frac{|\partial \Phi / \partial z|}{Q_{\infty}} \ll 1
$$

which, when applied to Equation (3.23), presents the following geometric restrictions:

$$
\left|\frac{\partial \eta}{\partial x}\right| \ll 1, \quad\left|\frac{\partial \eta}{\partial y}\right| \ll 1, \quad\left|\frac{W_{\infty}}{U_{\infty}}\right|=\tan \alpha \approx \alpha \ll 1
$$

The first restriction is that of a thin wing compared to the chord length. The second result, when considering small angles of attack $\alpha$, permits the approximations:

$$
W_{\infty} \approx Q_{\infty} \alpha, \quad U_{\infty} \approx Q_{\infty}
$$

which greatly reduces Equation (3.23):

$$
\frac{\partial \Phi}{\partial z}(x, y, z=\eta)=Q_{\infty}\left(\frac{\partial \eta}{\partial x}-\alpha\right)
$$

The thin wing approximation (first restriction of Equation (3.25)) requires that the boundary condition of Equation (3.27) be transfered to the $\mathrm{x}-\mathrm{y}$ plane. This is achieved through a Taylor series expansion of $\eta$ :

$$
\frac{\partial \Phi}{\partial z}(x, y, z=\eta)=\frac{\partial \Phi}{\partial z}(x, y, 0)+\eta \frac{\partial^{2} \Phi}{\partial z^{2}}(x, y, 0)+O\left(\eta^{2}\right)
$$

Staying consistent with the small-disturbance approximation, only the first term of the Taylor series expansion is used which yields the first-order boundary condition approximation

$$
\frac{\partial \Phi}{\partial z}(x, y, 0)=Q_{\infty}\left(\frac{\partial \eta}{\partial x}-\alpha\right)
$$

This illustrates the relation between the unknown potential, $\Phi$, and the known free-stream velocity, wing geometry, and angle of attack.

\subsection{Analytical Solution}

The analytical solution to the potential flow problem will be discussed in this section prior to introducing the numerical Panel Method. The linear boundary condition (Equation (3.29)) developed in the previous section is first specified for both the upper and lower surfaces of 
the wing using the expressions for the wing thickness $\eta_{t}$ and camberline $\eta_{c}$ introduced in Section 3.1.3:

$$
\begin{aligned}
& \frac{\partial \Phi}{\partial z}(x, y, 0+)=\left(\frac{\partial \eta_{c}}{\partial x}+\frac{\partial \eta_{t}}{\partial x}\right) Q_{\infty}-Q_{\infty} \alpha \\
& \frac{\partial \Phi}{\partial z}(x, y, 0+)=\left(\frac{\partial \eta_{c}}{\partial x}-\frac{\partial \eta_{t}}{\partial x}\right) Q_{\infty}-Q_{\infty} \alpha
\end{aligned}
$$

The boundary condition at infinity (Equation (3.6)) for just the perturbation potential $\Phi$ now becomes

$$
\lim _{r \rightarrow \infty} \nabla \Phi=0
$$

recalling from Equation (3.20) that this represents the velocity induced by the motion of the wing in a stationary frame of reference. Since the three boundary conditions (Equations (3.30) - (3.32)) and the continuity equation (Equation (3.22)) are all linear, the potential problem can be decomposed into three simpler problems. The solutions to the three subproblems can then be superimposed to obtain a complete solution to the potential flow problem.

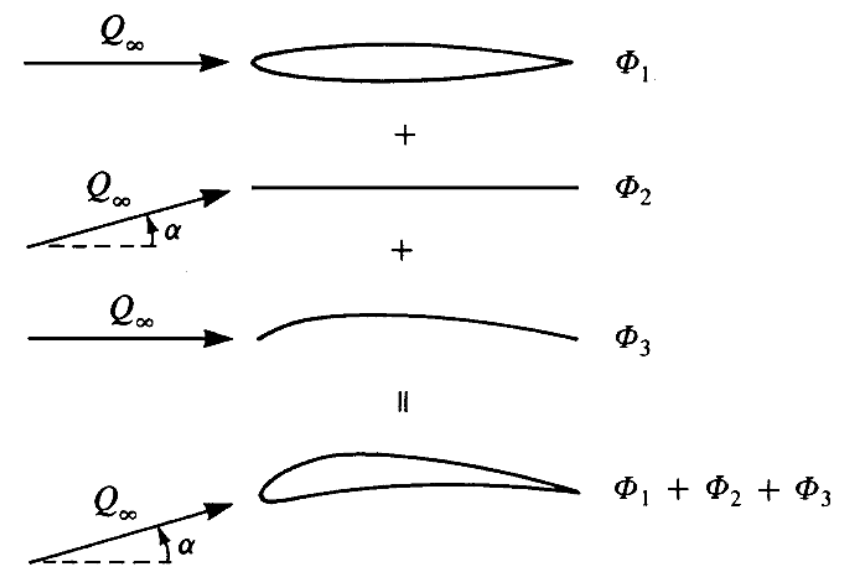

Figure 3.3: Decomposition of the thick cambered wing at an angle of attack into three simpler problems [20].

These three subproblems are:

1. Symmetric wing with nonzero thickness at zero angle of attack (thickness effect)

2. Zero-thickness, uncambered wing at angle of attack (angle of attack effect) 
3. Zero-thickness, cambered wing at zero angle of attack (camber effect)

and are shown schematically in Figure 3.3 and mathematically as

$$
\Phi=\Phi_{1}+\Phi_{2}+\Phi_{3}
$$

These solutions are obtained by using source and doublet modeling elements. The source elements model thickness effects of the wing, whereas the doublet elements model camber and angle of attack effects. Before the solutions to the three subproblems are outlined, a brief explanation of the source and doublet elements will be made.

\subsubsection{Source and Doublet Modeling Elements}

The previous sections have illustrated that the only unknown is the velocity potential $\Phi$. Provided in [20] is a formal derivation of the conservation of mass:

$$
\int_{c . s .} \mathbf{n} \cdot \mathbf{q} d S=\int_{c . v .} \nabla \cdot \mathbf{q} d V
$$

where the surface integral on the left side can be converted to a volume integral through the use of the Divergence Theorem (the steps of which are not included here for brevity). It will be shown that any incompressible and irrotational flow, assumptions made earlier, can be represented by a distribution of sources and doublets over the bounding surface $S$ of a volume region $V$ as shown in Figure 3.4.

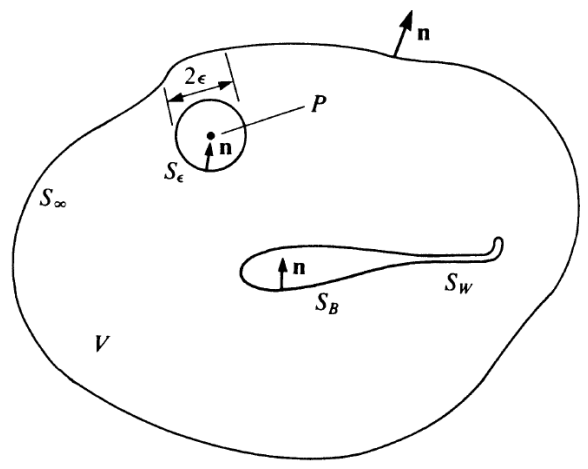

Figure 3.4: Nomenclature for the potential flow problem [20]. 
Replacing the vector appearing in Equation (3.34) with the vector

$$
\mathbf{q}=\Phi_{A} \nabla \Phi_{B}-\Phi_{B} \nabla \Phi_{A}
$$

produces one of Green's identities [20]:

$$
\int_{S}\left(\Phi_{A} \nabla \Phi_{B}-\Phi_{B} \nabla \Phi_{A}\right) \cdot \mathbf{n} d S=\int_{V}\left(\Phi_{A} \nabla^{2} \Phi_{B}-\Phi_{B} \nabla^{2} \Phi_{A}\right) d V
$$

The following relations are now used:

$$
\Phi_{A}=\frac{1}{r} \quad \text { and } \quad \Phi_{B}=\Phi
$$

Here, $\Phi_{B}$ is the velocity potential of the flow in $V$ (defined earlier in Section 3.1), and $r$ is the distance from a point $P(x, y, z)$ as depicted in Figure 3.4. The surface integral of Equation (3.36) includes the bounding surface of the wing $S_{B}$, a bounding surface of a wake model $S_{W}$ and the remaining infinite flow region bounded by $S_{\infty}$ :

$$
S=S_{B}+S_{W}+S_{\infty}
$$

The final term in Equation (3.36), $\Phi_{A}$, will be shown to represent the potential of a source element which will later be used to model thickness effects. Upon inspection, $\Phi_{A}$ is unbounded as $P$ is approached:

$$
\lim _{r \rightarrow 0} \Phi_{A}=\infty
$$

which requires that the point $P$ be excluded from the region of integration by surrounding it by a small sphere of radius $\epsilon$. In doing so (when point $P$ is outside of $V$ ), both $\Phi_{A}$ and $\Phi_{B}$ satisfy Laplace's equation (also the continuity equation introduced earlier):

$$
\nabla^{2}\left(\frac{1}{r}\right)=0 \quad \text { and } \quad \nabla^{2} \Phi_{B}=0
$$

Substituting the relations of Equation (3.37) into Equation (3.36) yields

$$
\int_{\mathrm{S}+\text { sphere } \epsilon}\left(\frac{1}{r} \nabla \Phi-\Phi \nabla \frac{1}{r}\right) \cdot \mathbf{n} d S=0
$$

To carry out the integral over the sphere, spherical coordinates are introduced at $P$ resulting in

$$
\mathbf{n}=-\mathbf{e}_{r}, \quad \mathbf{n} \cdot \nabla \Phi=\frac{\partial \Phi}{\partial r}, \quad \text { and } \quad \nabla\left(\frac{1}{r}\right)=-\left(\frac{1}{r^{2}}\right) \mathbf{e}_{r}
$$


which, when substituted into Equation (3.41) yields

$$
-\int_{\text {sphere } \epsilon}\left(\frac{1}{r} \frac{\partial \Phi}{\partial r}+\frac{\Phi}{r^{2}}\right) d S+\int_{S}\left(\frac{1}{r} \nabla \Phi-\Phi \nabla \frac{1}{r}\right) \cdot \mathbf{n} d S=0
$$

On the sphere surrounding $P, \int d S=4 \pi \epsilon^{2}$, and when $\epsilon \rightarrow 0$ the first term in the first integral goes to zero, while the second term becomes

$$
-\int_{\text {sphere } \epsilon}\left(\frac{\Phi}{r^{2}}\right) d S=-4 \pi \Phi(P)
$$

and allows Equation (3.43) to be rewritten as

$$
\Phi(P)=\frac{1}{4 \pi} \int_{S}\left(\frac{1}{r} \nabla \Phi-\Phi \nabla \frac{1}{r}\right) \cdot \mathbf{n} d S
$$

The potential flow at any point in the flow field can now be determined with the values of $\Phi$ and $\partial \Phi / \partial n$ on the boundaries of $S$.

For a moment, consider a point that lies within the boundary of $S_{B}$ which results in an "internal potential" $\Phi_{i}$. Because point $P$ is outside of $V$ Equation (3.41) can be applied:

$$
0=\frac{1}{4 \pi} \int_{S_{B}}\left(\frac{1}{r} \nabla \Phi_{i}-\Phi_{i} \nabla \frac{1}{r}\right) \cdot \mathbf{n} d S=0
$$

This expression for the internal potential can be added to the earlier potential expression (Equation (3.45))

$\Phi(P)=\frac{1}{4 \pi} \int_{S_{B}}\left[\frac{1}{r} \nabla\left(\Phi-\Phi_{i}\right)-\left(\Phi-\Phi_{i}\right) \nabla \frac{1}{r}\right] \cdot \mathbf{n} d S+\frac{1}{4 \pi} \int_{S_{W}+S_{\infty}}\left(\frac{1}{r} \nabla \Phi-\Phi \nabla \frac{1}{r}\right) \cdot \mathbf{n} d S$

which now expresses the potential at any point $P$ within the region $V$ in terms of the potential values on the bounding surfaces $S_{B}, S_{W}$ and $S_{\infty}$. Consider first the potential on the bounding surface $S_{B}$ and a portion of the surface as shown in Figure 3.5. The difference in the external and internal potentials are defined as

$$
-\mu=\Phi-\Phi_{i}
$$

with the difference between the normal derivatives expressed as

$$
-\sigma=\frac{\partial \Phi}{\partial n}-\frac{\partial \Phi_{i}}{\partial n}
$$


These elements are called doublet $(\mu)$ and source $(\sigma)$ with the minus signs a result of the direction of $\mathbf{n}$. Equation (3.47) can then be rewritten as

$$
\Phi(P)=-\frac{1}{4 \pi} \int_{S_{B}}\left[\sigma\left(\frac{1}{r}\right)-\mu \frac{\partial}{\partial n}\left(\frac{1}{r}\right)\right] d S+\frac{1}{4 \pi} \int_{S_{W}}\left[\mu \frac{\partial}{\partial n}\left(\frac{1}{r}\right)\right] d S+\Phi_{\infty}(P)
$$

with $\mathbf{n} \cdot \nabla$ replaced by $\partial / \partial n$ and $S_{\infty}$ taken far enough away to be replaced by $\Phi(P)$. The integral over $S_{W}$ is the potential difference between the upper and lower surfaces of the wake which is discussed in Section 4.1.2.

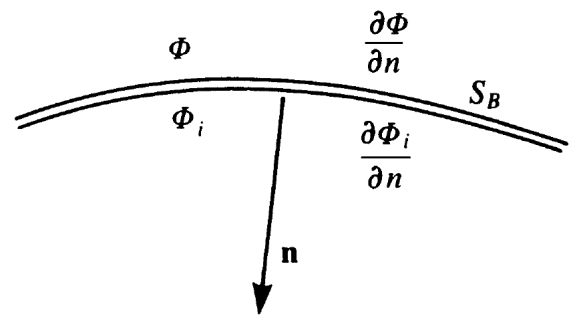

Figure 3.5: Velocity potential on bounding surface $S_{B}[20]$.

The bounding surface of interest has now been composed of the wing and wake. The source element has been introduced as having a dependence on the wing geometry (the outward normal vector), and the doublet element has been shown to be the difference between potentials. This allows the three subproblems to be solved using the bounded surface, the wing geometry and the difference between potentials, as is explained in the following subsections.

\subsubsection{Symmetric Wing with Nonzero Thickness at Zero Angle of Attack}

To analyze the effect of thickness consider the symmetric wing with thickness distribution $\eta_{t}(x)$ at zero angle of attack shown in Figure 3.6(a). Following from Equation (3.22) a solution to the equation

$$
\nabla^{2} \Phi_{1}=0
$$

is sought with the boundary condition at the $z=0$ plane given by

$$
\frac{\partial \Phi_{1}}{\partial z}(x, y, 0 \pm)= \pm \frac{\partial \eta_{t}}{\partial x} Q_{\infty}
$$




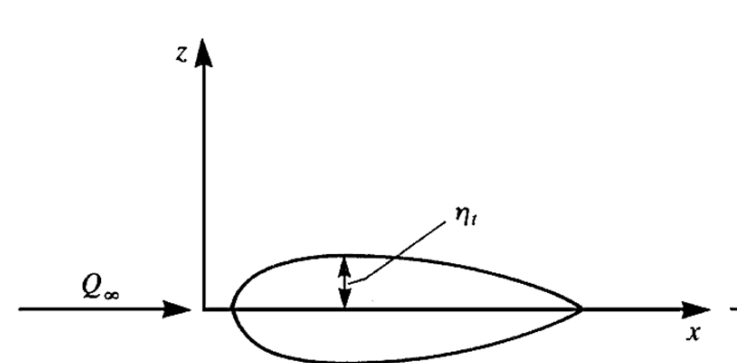

(a)

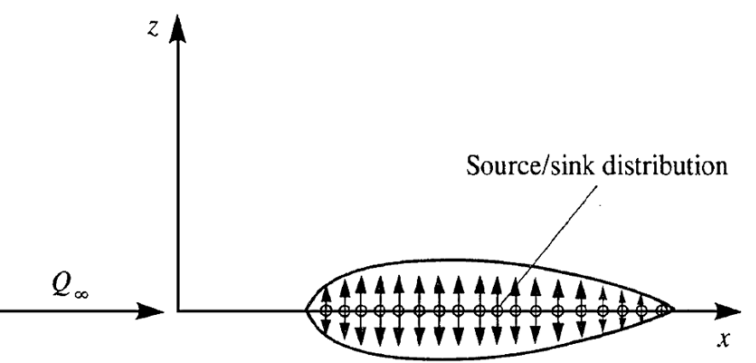

(b)

Figure 3.6: (a) Wing thickness function $\eta_{t}[20]$. (b) Modeling the thickness problem by a source distribution [20].

The symmetry of this subproblem allows the placement of a source element distribution at the wing section centreline to model the flow, as shown in Figure 3.6(b). The modeling source element, $\sigma$, was previously introduced. The effect that this source element has on the velocity potential at a point $(x, y, z)$ is given by

$$
\Phi=\frac{-\sigma}{4 \pi r}
$$

where $\mathrm{r}$ is the distance from the point singularity located at $\left(x_{0}, y_{0}, z_{0}\right)$ :

$$
r=\sqrt{\left(x-x_{0}\right)^{2}+\left(y-y_{0}\right)^{2}+\left(z-z_{0}\right)^{2}}
$$

The velocity potential at an arbitrary point $(x, y, z)$ due to the distribution of the source elements on the $x-y$ plane $(z=0)$ will be

$$
\Phi_{1}(x, y, z)=\frac{-1}{4 \pi} \int_{\text {wing }} \frac{\sigma\left(x_{0}, y_{0}\right) d x_{0} d y_{0}}{\sqrt{\left(x-x_{0}\right)^{2}+\left(y-y_{0}\right)^{2}+z^{2}}}
$$

This integration is only carried out over the wing and excludes the wake, a point which will be later revisited in the numerical approach. The current analytical approach, however, allows the solid-surface boundary condition to be examined by obtaining the normal velocity component $w(x, y, z)$ as the differentiation with respect to $z$ :

$$
w(x, y, z)=\frac{\partial \Phi_{1}}{\partial z}=\frac{z}{4 \pi} \int_{\text {wing }} \frac{\sigma\left(x_{0}, y_{0}\right) d x_{0} d y_{0}}{\left[\left(x-x_{0}\right)^{2}+\left(y-y_{0}\right)^{2}+z^{2}\right]^{3 / 2}}
$$

A limit process is used to find $w(x, y, 0)$ which results in

$$
w(x, y, 0 \pm)=\lim _{z \rightarrow 0 \pm} w(x, y, z)= \pm \frac{\sigma(x, y)}{2}
$$


where + and - denote the upper and lower surfaces of the wing, respectively. Equating this result to the boundary condition for this subproblem (Equation (3.52))

$$
\frac{\partial \Phi_{1}}{\partial z}(x, y, 0 \pm)= \pm \frac{\partial \eta_{t}}{\partial x} Q_{\infty}= \pm \frac{\sigma(x, y)}{2}
$$

yields an expression for the source strength:

$$
\sigma(x, y)=2 Q_{\infty} \frac{\partial \eta_{t}}{\partial x}(x, y)
$$

After substituting Equation (3.59) into Equation (3.55):

$$
\Phi_{1}(x, y, z)=\frac{-Q_{\infty}}{2 \pi} \int_{\text {wing }} \frac{\left[\partial \eta_{t}\left(x_{0}, y_{0}\right) / \partial x\right] d x_{0} d y_{0}}{\sqrt{\left(x-x_{0}\right)^{2}+\left(y-y_{0}\right)^{2}+z^{2}}}
$$

an expression to solve the first potential flow subproblem is obtained.

\subsubsection{Zero-Thickness Cambered Wing at Angle of Attack (Lifting Prob- lem)}

The angle of attack and camber effects are analyzed together by considering a wing with camber function $\eta_{c}(x, y)$ at angle of attack $\alpha$ as shown in Figure 3.7 (a).

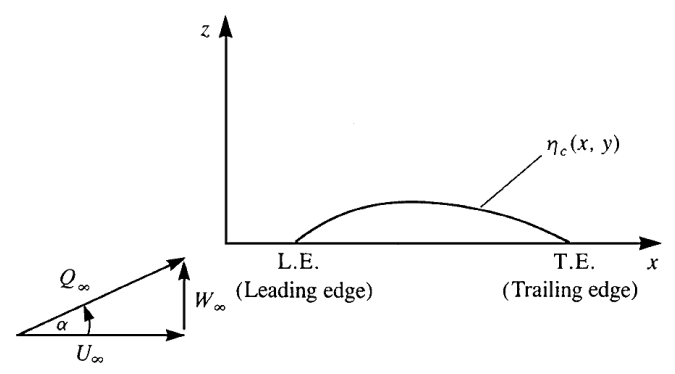

(a)

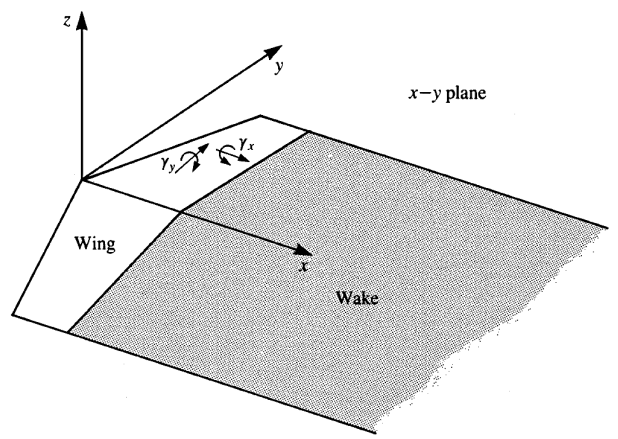

(b)

Figure 3.7: (a) Wing camber function $\eta_{c}[20]$. (b) Lifting-surface model of a threedimensional wing $[20]$.

Following from Equation (3.22) a solution to the equation

$$
\nabla^{2} \Phi_{2,3}, \nabla^{2} \Phi_{2}, \nabla^{2} \Phi_{3}=0
$$


is sought with the boundary condition at the $z=0$ plane given by

$$
\begin{aligned}
\frac{\partial \Phi_{2,3}}{\partial z}(x, y, 0 \pm) & =\frac{\partial \Phi_{2}}{\partial z}(x, y, 0 \pm)+\frac{\partial \Phi_{3}}{\partial z}(x, y, 0 \pm) \\
& =-Q_{\infty} \alpha+\frac{\partial \eta_{c}}{\partial x} Q_{\infty} \\
& =Q_{\infty}\left(\frac{\partial \eta_{c}}{\partial x}-\alpha\right)
\end{aligned}
$$

The antisymmetric nature of this subproblem (with respect to the $z$ direction) permits a solution through the use of a doublet distribution [20]. Doublet singularity elements are solutions to Equation (3.22) and satisfy the boundary condition of Equation (3.6). The potential due to a doublet element is given by

$$
\Phi=\frac{\mu}{4 \pi} \mathbf{n} \cdot \nabla\left(\frac{1}{r}\right)
$$

which if located at $\left(x_{0}, y_{0}, z_{0}\right)$, a velocity potential at a point $(x, y, z)$ is expressed as

$$
\Phi(x, y, z)=\frac{\mu}{4 \pi}\left(\begin{array}{c}
\frac{\partial}{\partial x} \\
\frac{\partial}{\partial y} \\
\frac{\partial}{\partial z}
\end{array}\right) \frac{1}{\sqrt{\left(x-x_{0}\right)^{2}+\left(y-y_{0}\right)^{2}+\left(z-z_{0}\right)^{2}}}
$$

The inclusion of angle of attack results in lift being generated by the wing and produces vortex lines which cannot begin and terminate in the fluid [20]. A wake region is included in the model to overcome this limitation. Due to the small-disturbance approximation, the wake is modeled as a planar region and placed on the $z=0$ plane (Figure $3.7(\mathrm{~b})$ ).

With lift being generated in the $z$ direction, differentiation of the doublet solution of Equation (3.64) is taken with respect to $z$. This produces doublets pointing in the $z$ direction and creates a pressure jump in this direction (as is expected to generate lift). The result of this differentiation then becomes

$$
\Phi(x, y, z)=\frac{-\mu\left(x_{0}, y_{0}\right)\left(z-z_{0}\right)}{4 \pi\left[\left(x-x_{0}\right)^{2}+\left(y-y_{0}\right)^{2}+\left(z-z_{0}\right)^{2}\right]^{3 / 2}}
$$

With a doublet distribution over the wing and wake (Figure 3.7(b)), the induced potential at an arbitrary point $(x, y, z)$ is

$$
\Phi(x, y, z)=\frac{1}{4 \pi} \int_{\text {wing+wake }} \frac{-\mu\left(x_{0}, y_{0}\right) z d x_{0} d y_{0}}{\left[\left(x-x_{0}\right)^{2}+\left(y-y_{0}\right)^{2}+z^{2}\right]^{3 / 2}}
$$


The velocity components on the wing surface are obtained by differentiating Equation (3.66) in the respective directions and letting $z \rightarrow 0$. Of interest is the normal velocity component in order to satisfy the boundary condition of Equation (3.62). The limit process in the $z$ direction is elaborate, but is given in [20]:

$$
\begin{aligned}
w(x, y, 0 \pm) & =\frac{\partial \Phi}{\partial z} \\
& =\frac{1}{4 \pi} \int_{\text {wing+wake }} \frac{\mu\left(x_{0}, y_{0}\right)}{\left(y-y_{0}\right)^{2}}\left[1+\frac{\left(x-x_{0}\right)}{\sqrt{\left(x-x_{0}\right)^{2}+\left(y-y_{0}\right)^{2}}}\right] d x_{0} d y_{0}
\end{aligned}
$$

Equating this result to the boundary condition of this subproblem (Equation (3.62)) results in an expression for the unknown doublet strength $\mu(x, y)$ :

$$
\frac{1}{4 \pi} \int_{\text {wing+wake }} \frac{\mu\left(x_{0}, y_{0}\right)}{\left(y-y_{0}\right)^{2}}\left[1+\frac{\left(x-x_{0}\right)}{\sqrt{\left(x-x_{0}\right)^{2}+\left(y-y_{0}\right)^{2}}}\right] d x_{0} d y_{0}=Q_{\infty}\left(\frac{\partial \eta_{c}}{\partial x}-\alpha\right)
$$

Solving for the unknown doublet strength in Equation (3.68) leads to the velocity distribution which allows for secondary computations to be made which includes the pressure distribution and the required aerodynamic loads.

\subsubsection{Superposition of the Problems}

With two subproblems examined, the superposition of their solutions are:

$$
\Phi(x, y, z)=\Phi_{1}+\Phi_{2,3}=\frac{-1}{4 \pi} \int_{\text {wing }} \sigma\left(\frac{1}{r}\right) d S+\frac{1}{4 \pi} \int_{\text {wing }+ \text { wake }} \mu \mathbf{n} \cdot \nabla\left(\frac{1}{r}\right) d S
$$

Here, the potential is a function of the source element strength, the doublet element strength, and the wing geometry. This will be the basis of the numerical Panel Method which is the focus of Chapter 4. 


\section{Chapter 4}

\section{Panel Method: Numerical Approach}

The superposition of three cases were used in Chapter 3 to analytically solve the general problem of an arbitrary airfoil. In setting up this approach thin-airfoil theory was used but it has a distinct limitation in the validity of the solution to this problem as it only applies to thin-airfoils, disregards any interactions between multiple surfaces (i.e. fuselage and wing interfaces), and boundary conditions are applied at the airfoil chord [20]. These limitations are all overcome with the application of a numerical technique commonly referred to as a Panel Method and has been used extensively to solve realistic potential flow problems [22]. The analytical method was introduced first as many derivations and concepts do carry over and are used in the numerical solution that allows realistic geometries to be introduced which can prove to be quite complex using analytical methods. Additionally, the boundary conditions imposed on analytical results can be made more realistic by applying them at the boundary surfaces of the realistic geometry through the use of a discretized paneling method (hence, the name). This combination of realistic geometry and boundary conditions allows the potential flow problem to be applied to a variety of situations including that of three-dimensional airfoil in a two-dimensional flow field.

This chapter focuses on describing the numerical Panel Method and its use in solving the general problem, Equation (3.69), that concluded Chapter 3. The theory outlined in this chapter is adapted from [20] unless otherwise stated. First, the analytical method is used to explain and apply boundary conditions to generate a unique solution to the

general problem. This is followed by a description of the previously-introduced source and 
doublet modeling elements in a way that allows numerical integration to take place. The actual discretization of the wing surface is then explained. The descriptions of the source and doublet elements are then used to introduce their influence coefficients and reduce the problem to a set of linear algebraic equations that, when solved, provide the velocity potential of a three-dimensional wing in a two-dimensional flow. Finally, the computation of the pressure and lifting loads is provided. This process is depicted in Figure 4.1.

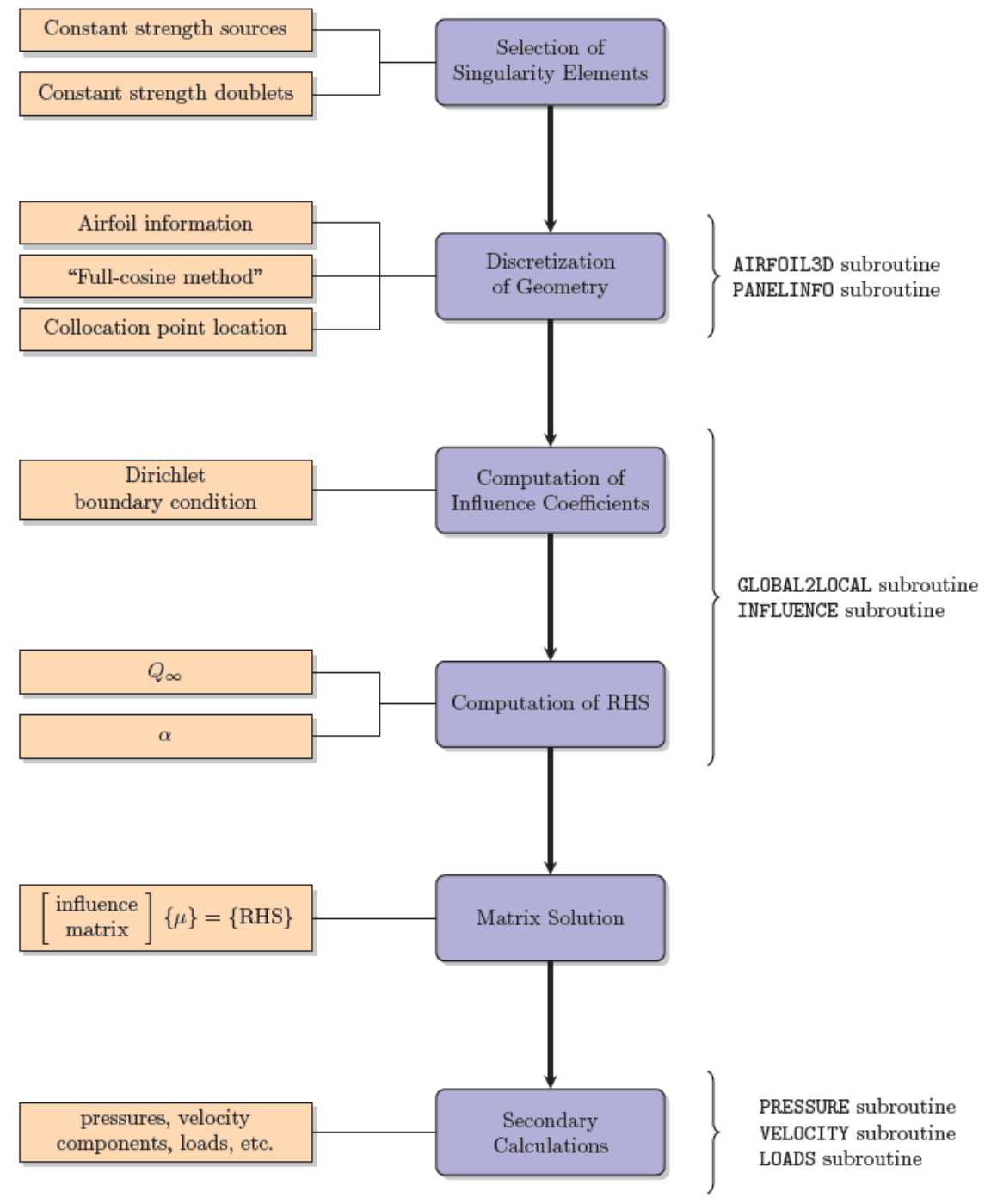

Figure 4.1: Flowchart of the numerical approach 


\subsection{Boundary Conditions}

To develop the numerical Panel Method, the general solution to the lifting problem is used and is now expressed in terms of the total potential $\Phi^{*}$

$$
\nabla^{2} \Phi^{*}=0
$$

The use of the total potential allows for the scenario depicted in Figure 4.2 where the flow of interest lies in a region $V$ outside of a submerged body bounded by $S_{B}$. The previous chapter introduced the use of source and doublet elements in the general solution to Equation (3.5). In that solution the elements were placed on the chordline ( $z=0$ plane). To solve Equation (4.1) these elements are now placed on the boundary $S_{B}$ :

$$
\Phi^{*}(x, y, z)=\frac{1}{4 \pi} \int_{\text {wing }+ \text { wake }} \mu \mathbf{n} \cdot \nabla\left(\frac{1}{r}\right) d S-\frac{-1}{4 \pi} \int_{\text {wing }} \sigma\left(\frac{1}{r}\right) d S+\Phi_{\infty}
$$

The vector $\mathbf{n}$ is positive outside of $V$ and is normal to $S_{B}$ (direction of the potential jump $\mu$ ), and $\Phi_{\infty}$ is the free-stream potential

$$
\Phi_{\infty}=U_{\infty} x+V_{\infty} y+W_{\infty} z
$$

Upon inspection of Equation (4.2) the seemingly infinite combinations of source and doublet distributions poses a problem when trying to acquire a single solution. This problem is overcome by setting limitations on the source and doublet distributions by revisiting the analytical approach presented earlier where source elements were used to simulate the effect of thickness and doublet elements were used to simulate the antisymmetric lifting effect.

\subsubsection{Zero Normal Flow Condition}

The physically-intuitive condition of zero flow normal to the surface was introduced in Section 3.1.2. Assuming no flow through the surface $S_{B}$ implies that the internal potential $\Phi_{i}^{*}$ does not change or,

$$
\Phi_{i}^{*}=\text { constant }
$$


Setting this constant to zero and using this condition on the surface of $S_{B}$ is called the Dirichlet Boundary Condition [20]:

$$
\Phi_{i}^{*}(x, y, z)=\frac{1}{4 \pi} \int_{\text {wing }+ \text { wake }} \mu \mathbf{n} \cdot \nabla\left(\frac{1}{r}\right) d S-\frac{-1}{4 \pi} \int_{\text {wing }} \sigma\left(\frac{1}{r}\right) d S+\Phi_{\infty}=0
$$

For a moment, consider the scenario depicted in Figure 4.2 and Equation (4.5) both using ground-fixed coordinates, which implies that $\Phi_{\infty}=0$. This reduces Equation (4.5) to

$$
\Phi_{i}^{*}(x, y, z)=\frac{1}{4 \pi} \int_{\text {wing }+ \text { wake }} \mu \mathbf{n} \cdot \nabla\left(\frac{1}{r}\right) d S-\frac{-1}{4 \pi} \int_{\text {wing }} \sigma\left(\frac{1}{r}\right) d S=0
$$

Upon introducing the source as the difference between the normal derivatives in Equation (3.49), it follows that

$$
-\sigma=\frac{\partial \Phi^{*}}{\partial n}-\frac{\partial \Phi_{i}^{*}}{\partial n}
$$

and Equation 4.6 requires that

$$
\frac{\partial \Phi_{i}^{*}}{\partial n}=0
$$

Consequently, for Equation (4.6) and the definition of Equation (4.7) to be valid the value of the source strength is required to be

$$
\sigma=\mathbf{n} \cdot \mathbf{Q}_{\infty}
$$

where $\mathbf{n}$ points into the body as shown in Figure 4.2 as interest is focused on the internal potential.

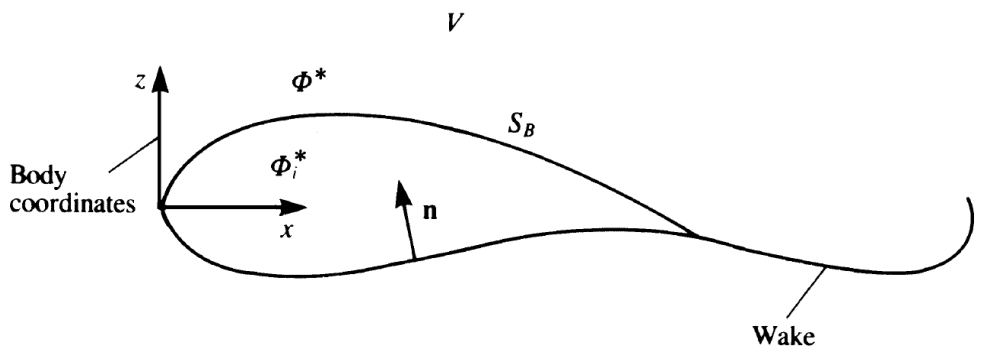

Figure 4.2: Potential flow over a closed body [20]. 


\subsubsection{Wake Condition}

Upon introducing the doublet element in Section 3.2.1, integrals were taken over both the body and the wake. This was due to the antisymmetric nature of the lifting problem, which is depicted in Figure 4.3. Here, the doublet distribution that comprises the wake $\left(\mu_{W}\right)$ must be related to the upper $\left(\mu_{U}\right)$ and lower $\left(\mu_{L}\right)$ doublet strengths at the trailing edge (T.E.) in a way that eliminates any rotationality which would contradict the earlier assumption of an irrotational fluid. By applying a condition (the Kutta condition) along the trailing edge, a relationship between the trailing edge and wake doublet strengths is formed:

$$
\mu_{T . E .}=\text { constant }=\mu_{W}
$$

and in terms of the upper and lower surfaces,

$$
\mu_{U}-\mu_{L}-\mu_{W}=0
$$

which provides a condition on the doublet strengths to further the solution of Equation (4.2).

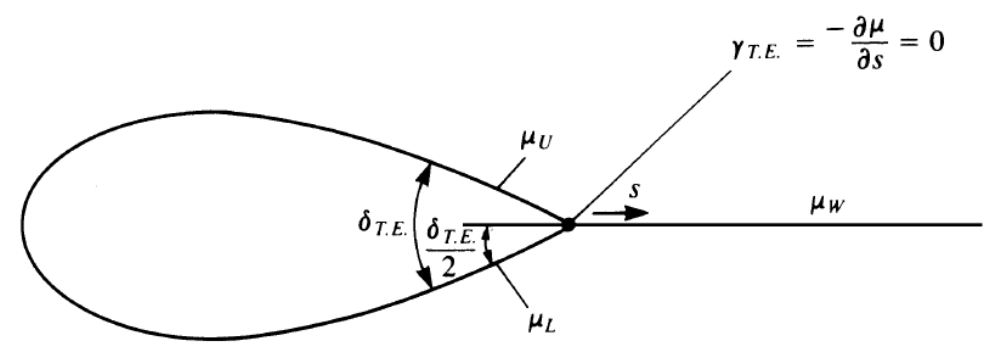

Figure 4.3: Kutta condition using a surface doublet distribution [20].

\subsection{Modeling Elements}

The zero normal flow condition and a condition imposed on the wake at the trailing edge of the wing provide unique conditions on the source and doublet strengths respectively which will result in a unique solution to the lifting problem. Equation (4.6) is the problem to solve 
and first requires a slight transformation:

$$
\begin{aligned}
\sum_{k=1}^{N} \frac{1}{4 \pi} \int_{\text {body panel }} \mu \mathbf{n} \cdot \nabla\left(\frac{1}{r}\right) d S & +\sum_{l=1}^{N_{W}} \frac{1}{4 \pi} \int_{\text {wake panel }} \mu \mathbf{n} \cdot \nabla\left(\frac{1}{r}\right) d S \\
& -\sum_{k=1}^{N} \frac{1}{4 \pi} \int_{\text {body panel }} \sigma\left(\frac{1}{r}\right) d S=0
\end{aligned}
$$

Each panel consists of a constant strength source and doublet distribution which creates an influence on all points $P$ along the wing surface. The goal now becomes to sum the influence of all $k$ body panels and $l$ wake panels for each point $P$. It will shortly be shown that these influences are functions of the panels' geometry and the location of point $P$ as shown in Figure 4.4 .

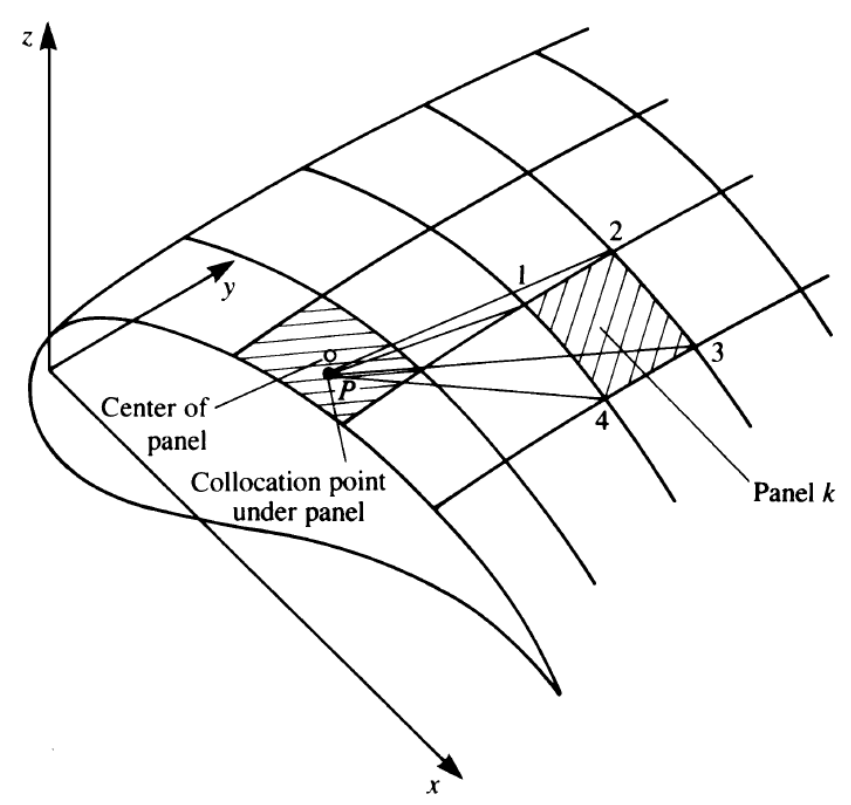

Figure 4.4: The influence that panel $k$ has on collocation point $P[20]$.

\subsubsection{Three-Dimensional Constant Strength Source Element}

Shown in Figure 4.5 is a surface element bounded by corner points $\left(x_{1}, y_{1}, z_{1}\right), \cdots,\left(x_{4}, y_{4}, z_{4}\right)$. This surface element contains a constant strength source distribution $\sigma$ per area defined by the four corner points. Following from Equations (3.53)(3.55), the potential at any point $P(x, y, z)$ (in this case the collocation points of interest 
over the wing surface) due to this source distribution is given by

$$
\Phi_{\text {source }}(x, y, z)=\frac{-\sigma}{4 \pi} \int_{S} \frac{d S}{\sqrt{\left(x-x_{0}\right)^{2}+\left(y-y_{0}\right)^{2}+z^{2}}}
$$

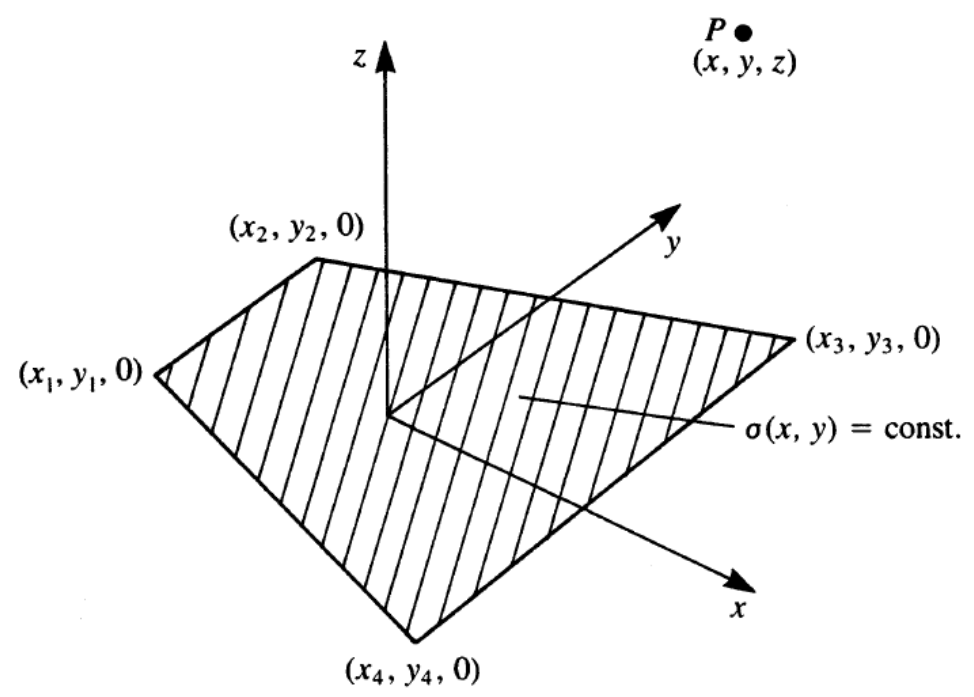

Figure 4.5: Constant strength source element [20].

Fortunately, this lengthy integration has already been performed and the results are provided in [20], [23], and [24]:

$$
\begin{aligned}
\Phi_{\text {source }}= & \frac{-\sigma}{4 \pi}\left\{\left[\frac{\left(x-x_{1}\right)\left(y_{2}-y_{1}\right)-\left(y-y_{1}\right)\left(x_{2}-x_{1}\right)}{d_{12}} \ln \frac{r_{1}+r_{2}+d_{12}}{r_{1}+r_{2}-d_{12}}\right.\right. \\
& +\frac{\left(x-x_{2}\right)\left(y_{3}-y_{2}\right)-\left(y-y_{2}\right)\left(x_{3}-x_{2}\right)}{d_{23}} \ln \frac{r_{2}+r_{3}+d_{23}}{r_{2}+r_{3}-d_{23}} \\
& +\frac{\left(x-x_{3}\right)\left(y_{4}-y_{3}\right)-\left(y-y_{3}\right)\left(x_{4}-x_{3}\right)}{d_{34}} \ln \frac{r_{3}+r_{4}+d_{34}}{r_{3}+r_{4}-d_{34}} \\
& \left.+\frac{\left(x-x_{4}\right)\left(y_{1}-y_{4}\right)-\left(y-y_{4}\right)\left(x_{1}-x_{4}\right)}{d_{41}} \ln \frac{r_{4}+r_{1}+d_{41}}{r_{4}+r_{1}-d_{41}}\right] \\
& -|z|\left[\tan ^{-1}\left(\frac{m_{12} e_{1}-h_{1}}{z r_{1}}\right)-\tan ^{-1}\left(\frac{m_{12} e_{2}-h_{2}}{z r_{2}}\right)\right. \\
& +\tan ^{-1}\left(\frac{m_{23} e_{2}-h_{2}}{z r_{2}}\right)-\tan ^{-1}\left(\frac{m_{23} e_{3}-h_{3}}{z r_{3}}\right) \\
& +\tan ^{-1}\left(\frac{m_{34} e_{3}-h_{3}}{z r_{3}}\right)-\tan ^{-1}\left(\frac{m_{34} e_{4}-h_{4}}{z r_{4}}\right) \\
& \left.\left.+\tan ^{-1}\left(\frac{m_{41} e_{4}-h_{4}}{z r_{4}}\right)-\tan ^{-1}\left(\frac{m_{41} e_{1}-h_{1}}{z r_{1}}\right)\right]\right\}
\end{aligned}
$$


where

$$
\begin{aligned}
& d_{12}=\sqrt{\left(x_{2}-x_{1}\right)^{2}+\left(y_{2}-y_{1}\right)^{2}} \\
& d_{23}=\sqrt{\left(x_{3}-x_{2}\right)^{2}+\left(y_{3}-y_{2}\right)^{2}} \\
& d_{34}=\sqrt{\left(x_{4}-x_{3}\right)^{2}+\left(y_{4}-y_{3}\right)^{2}} \\
& d_{41}=\sqrt{\left(x_{1}-x_{4}\right)^{2}+\left(y_{1}-y_{4}\right)^{2}}
\end{aligned}
$$

and

$$
\begin{aligned}
m_{12} & =\frac{y_{2}-y_{1}}{x_{2}-x_{1}} \\
m_{23} & =\frac{y_{3}-y_{2}}{x_{3}-x_{2}} \\
m_{34} & =\frac{y_{4}-y_{3}}{x_{4}-x_{3}} \\
m_{41} & =\frac{y_{1}-y_{4}}{x_{1}-x_{4}}
\end{aligned}
$$

and

$$
\begin{aligned}
& r_{k}=\sqrt{\left(x-x_{k}\right)^{2}+\left(y-y_{k}\right)^{2}+z^{2}}, \quad k=1,2,3,4 \\
& e_{k}=\left(x-x_{k}\right)^{2}+z^{2}, \quad k=1,2,3,4 \\
& h_{k}=\left(x-x_{k}\right)\left(y-y_{k}\right), \quad k=1,2,3,4
\end{aligned}
$$

By differentiating the velocity potential the velocity components can be obtained,

$$
(u, v, w)=\left(\frac{\partial \Phi_{\text {source }}}{\partial x}, \frac{\partial \Phi_{\text {source }}}{\partial y}, \frac{\partial \Phi_{\text {source }}}{\partial z}\right)
$$

Of importance, due to the zero normal flow condition, is the normal velocity component $w$. In particular, the value of this normal velocity is required when the point of interest becomes very close to the surface element $w(z \rightarrow 0)$. This value is given in [20] as

$$
w(z=0 \pm)=\frac{ \pm \sigma}{2}
$$

a relation that is revisited shortly. 


\subsubsection{Three-Dimensional Constant Strength Doublet Element}

Shown in Figure 4.6 is a surface element, again, bounded by corner points $\left(x_{1}, y_{1}, z_{1}\right), \cdots,\left(x_{4}, y_{4}, z_{4}\right)$. This surface element contains a constant strength doublet distribution $\mu$ directed in the $z$ direction.

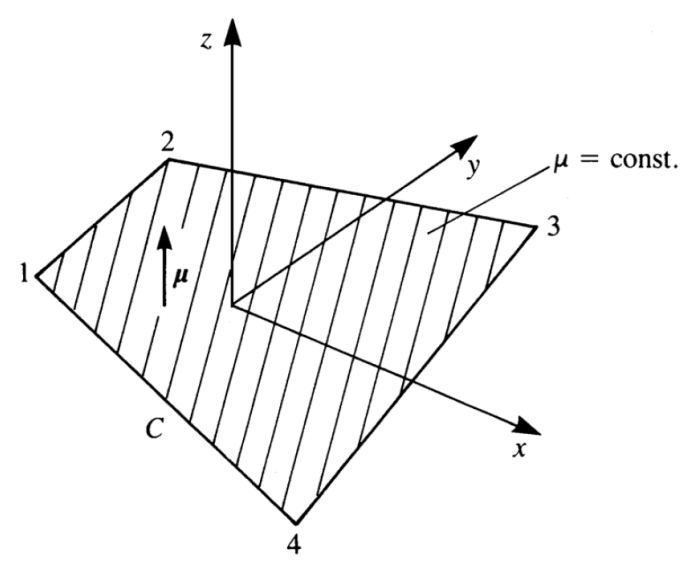

Figure 4.6: Constant strength doublet element [20].

Following from Equations (3.63)-(3.65), the potential at a point $P(x, y, z)$ due to this doublet distribution is given by

$$
\Phi_{\text {doublet }}(x, y, z)=\frac{-\mu}{4 \pi} \int_{S} \frac{z d S}{\left[\left(x-x_{0}\right)^{2}+\left(y-y_{0}\right)^{2}+z^{2}\right]^{3 / 2}}
$$

Once again, this integration has been performed and the results provided in [20], [23], and [24]:

$$
\begin{aligned}
\Phi_{\text {doublet }}= & \frac{-\mu}{4 \pi}\left[\tan ^{-1}\left(\frac{m_{12} e_{1}-h_{1}}{z r_{1}}\right)-\tan ^{-1}\left(\frac{m_{12} e_{2}-h_{2}}{z r_{2}}\right)\right. \\
& +\tan ^{-1}\left(\frac{m_{23} e_{2}-h_{2}}{z r_{2}}\right)-\tan ^{-1}\left(\frac{m_{23} e_{3}-h_{3}}{z r_{3}}\right) \\
& +\tan ^{-1}\left(\frac{m_{34} e_{3}-h_{3}}{z r_{3}}\right)-\tan ^{-1}\left(\frac{m_{34} e_{4}-h_{4}}{z r_{4}}\right) \\
& \left.+\tan ^{-1}\left(\frac{m_{41} e_{4}-h_{4}}{z r_{4}}\right)-\tan ^{-1}\left(\frac{m_{41} e_{1}-h_{1}}{z r_{1}}\right)\right]
\end{aligned}
$$

And noting that as $z \rightarrow 0$

$$
\Phi_{\text {doublet }}(z= \pm 0)=\mp \frac{\mu}{2}
$$


At this point expressions have been provided for the influences of source and doublet distributions as functions of the four corner points defining the surface elements and a single point at which the influence was sought $(P)$. These surface elements will now compose the wing surface for which the lifting problem is solved. The process of doing so is the focus of the next section.

\subsection{Discretization of the Wing into Panels}

Before the two conditions introduced in the previous section can be applied to the source and doublet strengths, a discretization process must first be carried out on the wing. This discretization produces constant-strength rectilinear panels as shown in Figure 4.7 which approximate the surface of the wing with $N$ surface panels and the wake with $N_{W}$ wake panels. Each panel has an associated collocation point $P$ at which the Dirichlet Boundary condition will be applied.

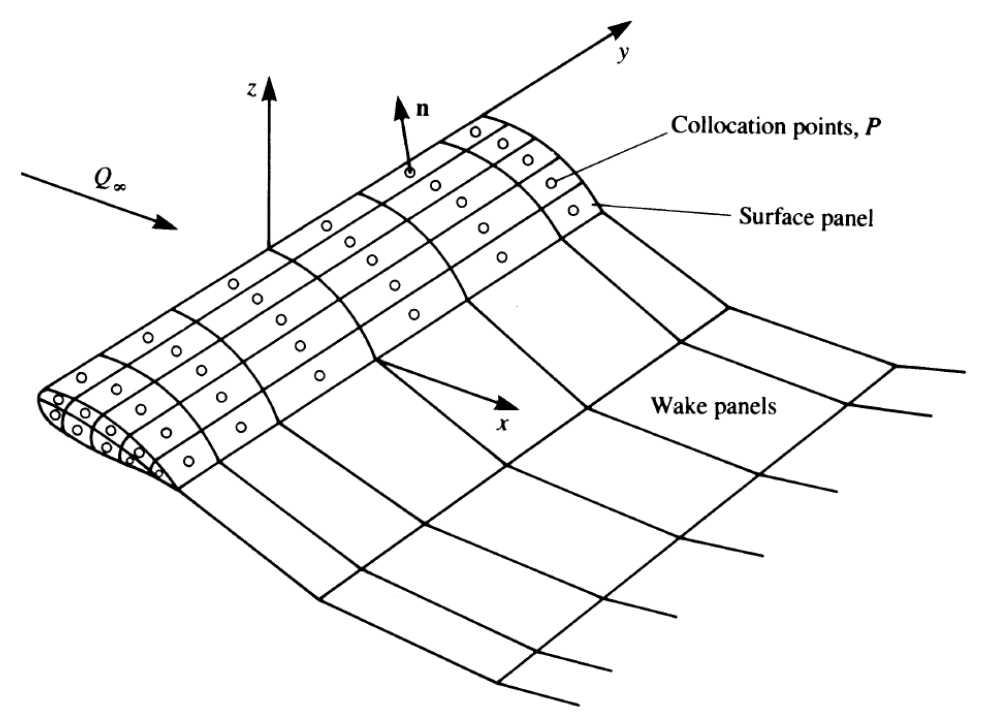

Figure 4.7: Paneling of the wing surface [20].

Division of the wing surface is done through a number of chord-wise and span-wise panels to create the three-dimensional discretization. This process is most easily explained by first considering a two-dimensional wing where only a chord-wise paneling process is used. With 
significant changes in flow expected to occur near the leading edge and trailing edge of the airfoil, there is more interest in these areas. To accommodate this, more panels are created at the leading and trailing edges using a "full-cosine" method of paneling. Shown in Figure 4.8, as an example, is a semicircle divided into 10 components which will divide the chord $c$ into 10 respective sections. The locations of these divisions, each described by a length $x$ from the leading edge, is determined using the spacing formula:

$$
x=\frac{c}{2}(1-\cos \beta)
$$

where $\Delta \beta=\pi / 10$ in this particular case.

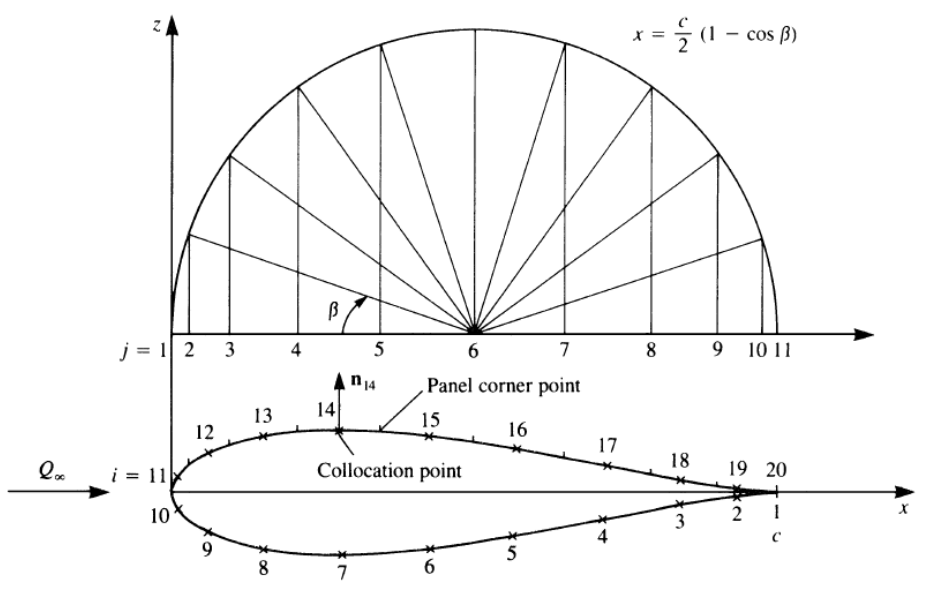

Figure 4.8: Method of two-dimensional paneling using the "full-cosine" method [20].

With the $x$-axis now divided into $10(N)$ panels, the location of each of the $(N+1)$ panel corner points can be determined as $\left(x_{j=1}, z_{j=1}\right),\left(x_{j=2}, z_{j=2}\right), \cdots,\left(x_{j=N+1}, z_{j=N+1}\right)$ and by using known airfoil geometry. The collocation points, denoted by x's in Figure 4.8, are placed at the centre of the panels and these locations can also be determined. It is at the collocation points that the boundary conditions will be applied. Figure 4.8 also depicts a panel outward normal vector $\mathbf{n}$ which will be used to describe the orientation of each panel in addition to its location.

Figure 4.9 illustrates the expansion of this two-dimensional example to three dimensions. The wing surface panels can be visualized as being unfolded into a single sheet where the 


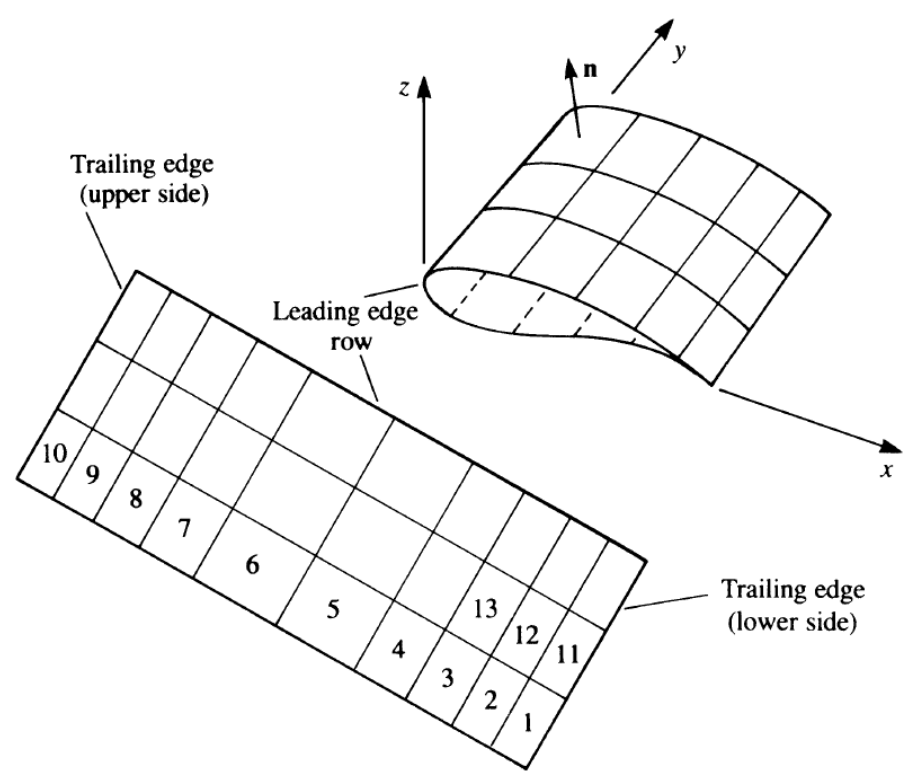

Figure 4.9: Three-dimensional paneling of the wing surface [20].

shown paneling number identification is used. Each rectilinear panel will have associated corner point locations, an outward normal vector $\mathbf{n}$ that describes its orientation, and a collocation point at the centre of the panel at which the boundary conditions will be applied.

\subsection{Influence Coefficients}

Any point $P$ on the wing surface will be influenced by the source and doublet distributions from the $N$ wing and $N_{W}$ wake panels. To obtain influence information as point $P$ is systematically placed along the wing surface an organized method of storing this information is needed. As such, the influences of a constant strength source element $\sigma$ and of a constant strength doublet element $\mu$ at a point $P$ are given in [20] respectively as:

$$
\begin{gathered}
\left.\frac{1}{4 \pi} \int_{1,2,3,4} \frac{\partial}{\partial n}\left(\frac{1}{r}\right) d S\right|_{k} \equiv C_{k} \quad\left(C_{l} \text { with wake panels }\right) \\
\left.\frac{-1}{4 \pi} \int_{1,2,3,4}\left(\frac{1}{r}\right) d S\right|_{k} \equiv B_{k}
\end{gathered}
$$


Like the surface elements described in Sections 4.2.1 and 4.2.2, the panels are defined using their corner points $1,2,3$, and 4 . By first performing these integrations, influence coefficients $B_{k}, C_{k}$ and $C_{l}$ are obtained:

$$
\left(\begin{array}{c}
x_{P}, y_{P}, z_{P} \\
x_{1}, y_{1}, z_{1} \\
x_{2}, y_{2}, z_{2} \\
x_{3}, y_{3}, z_{3} \\
x_{4}, y_{4}, z_{4} \\
\mu \text { or } \sigma
\end{array}\right) \Rightarrow\left(\begin{array}{c}
\text { influence } \\
\text { coefficient }
\end{array}\right) \Rightarrow(\Delta \Phi)_{P}
$$

That is, the influence experienced at a point $P$ as a result of the source distributions, the doublet distributions on the wing, and the doublet distributions on the wake are stored as influence coefficients $B_{k}, C_{k}$ and $C_{l}$ respectively. With the initial calculation of the influence coefficients, one final transformation of Equation (4.12) can occur,

$$
\sum_{k=1}^{N} C_{k} \mu_{k}+\sum_{l=1}^{N_{W}} C_{l} \mu_{l}+\sum_{k=1}^{N} B_{k} \sigma_{k}=0
$$

for which this procedure will have to be performed for each point $P$.

\subsection{Creating a Set of Linear Algebraic Equations}

By recalling the zero normal flow condition imposed on the solution (Equation (4.9)), allows the second set of known values to be included in Equation (4.35) (the first set of known values are the influence coefficients)

$$
\sigma_{k}=\mathbf{n}_{k} \cdot Q_{\infty}
$$

The panel locations and orientations provide known values for all panel normal vectors n. Additionally, through the combination of the determined airspeed (Chapter 6) and the 
aircraft pitch data from the ArduPilot (described in Chapter 5), a free stream velocity $Q_{\infty}$ is also known. Moving known values to their own side Equation (4.35) can be written as

$$
\sum_{k=1}^{N} C_{k} \mu_{k}+\sum_{l=1}^{N_{W}} C_{l} \mu_{l}=-\sum_{k=1}^{N} B_{k} \sigma_{k}
$$

To simplify the above equation, the two summation terms on the left-hand side will now be combined. Recalling from Equation (4.11), the strength of a doublet in the wake is used to apply the irrotational condition using the upper and lower surfaces of the trailing edge as shown in Figure 4.10.

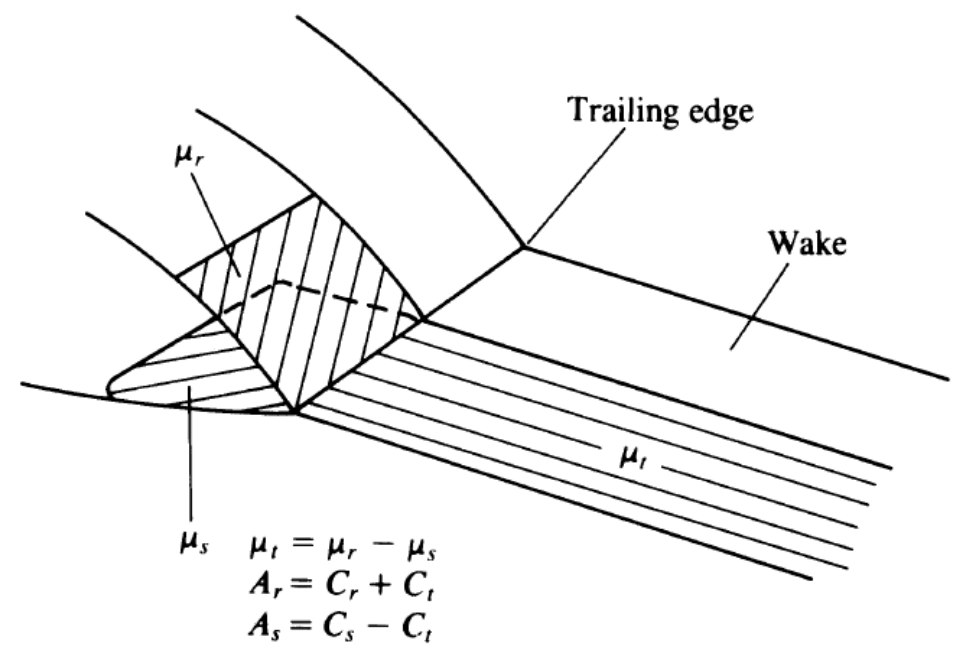

Figure 4.10: Wake doublet strength definition using trailing upper and lower panel doublet strengths $[20]$.

Here, the subscripts $r, s$, and $t$ are used to refer to the upper, lower, and wake doublets respectively. The relation between these surface panel doublet strengths is given by

$$
\mu_{t}=\mu_{r}-\mu_{s}
$$

which yields the influence of the wake element as

$$
C_{t} \mu_{t}=C_{t}\left(\mu_{r}-\mu_{s}\right)
$$


This relation combines the first two summation terms in Equation (4.37) such that

$$
\begin{gathered}
A_{k}=C_{k} \quad \text { if the panel is not at the trailing edge } \\
A_{k}=C_{k} \pm C_{t} \text { if the panel is at the trailing edge }
\end{gathered}
$$

and the algebraic equation then becomes

$$
\sum_{k=1}^{N} A_{k} \mu_{k}=-\sum_{k=1}^{N} B_{k} \sigma_{k}
$$

An organizational note must now be made to remain consistent throughout the remainder of the explanation of the Panel Method. The counter $k$ has been used to refer to the panels whose influences are being examined. An additional counter must be introduced to refer to the collocation point $P$ at which those influences are being examined, which now creates the algebraic equation:

$$
\left(\begin{array}{c}
a_{11}, a_{12}, \cdots, a_{1 N} \\
a_{21}, a_{22}, \cdots, a_{2 N} \\
\vdots \\
a_{N 1}, a_{N 2}, \cdots, a_{N N}
\end{array}\right)\left(\begin{array}{c}
\mu_{1} \\
\mu_{2} \\
\vdots \\
\mu_{N}
\end{array}\right)=-\left(\begin{array}{c}
b_{11}, b_{12}, \cdots, b_{1 N} \\
b_{21}, b_{22}, \cdots, b_{2 N} \\
\vdots \\
\vdots \\
b_{N 1}, b_{N 2}, \cdots, b_{N N}
\end{array}\right)\left(\begin{array}{c}
\sigma_{1} \\
\sigma_{2} \\
\vdots \\
\sigma_{N}
\end{array}\right)
$$

As an example, the coefficient $a_{42}$ refers to the influence that the $2^{\text {nd }}$ doublet panel has on the $4^{\text {th }}$ collocation point $P$. While providing an expression for the influence of a doublet element in Section 4.2.2, a value was also provided when the point $P$ approached the surface element. This is analogous to an influence being examined on the same panel at which the influence is being created, or in other words, $a_{k k}=1 / 2$. With a set of $N$ linear equations for the $N$ unknown $\mu_{k}$ terms (since the $\sigma_{k}$ terms are already known using Equation (4.36)) 
the right-hand-side matrix multiplication can be carried out resulting in

$$
\left(\begin{array}{c}
a_{11}, a_{12}, \cdots, a_{1 N} \\
a_{21}, a_{22}, \cdots, a_{2 N} \\
\vdots \\
a_{N 1}, a_{N 2}, \cdots, a_{N N}
\end{array}\right)\left(\begin{array}{c}
\mu_{1} \\
\mu_{2} \\
\vdots \\
\mu_{N}
\end{array}\right)=\left(\begin{array}{c}
\mathrm{RHS}_{1} \\
\mathrm{RHS}_{2} \\
\vdots \\
\mathrm{RHS}_{N}
\end{array}\right)
$$

which can now be solved.

\subsection{Secondary Calculations}

With the doublet strengths $\mu_{1, N}$ now solved for, the surface velocities, pressure coefficients, and lifting load can be determined. Local surface velocities are calculated using the local coordinates depicted in Figure 4.11.

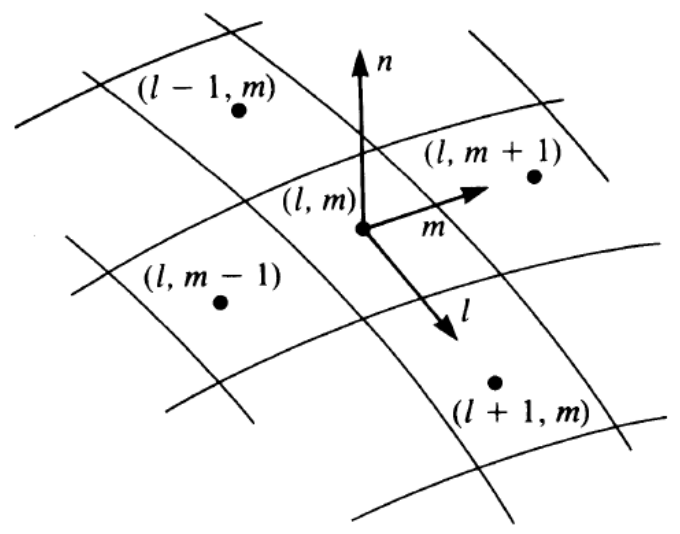

Figure 4.11: Local coordinate axes used to determine surface velocities [20].

The normal velocity component has already been determined through the zero normal flow condition,

$$
q_{n}=\sigma
$$


whereas the tangential components are given by a differentiation in the component directions,

$$
q_{l}=-\frac{\partial \mu}{\partial l}, \quad q_{m}=-\frac{\partial \mu}{\partial m}
$$

These are evaluated numerically using a central difference method where, as an example, the component in the $l$ direction for an arbitrary panel is

$$
q_{l}=\frac{1}{2 \Delta l}\left(\mu_{l-1}-\mu_{l+1}\right)
$$

given that $\Delta l$ is the panel length in the $l$ direction. The local velocities are then combined with the known free-stream velocities to obtain the total velocity

$$
\mathbf{Q}_{k}=\left(Q_{\infty l}, Q_{\infty m}, Q_{\infty n}\right)_{k}+\left(q_{l}, q_{m}, q_{n}\right)_{k}
$$

which is used to determine the pressure coefficient,

$$
C_{p k}=1-\frac{Q_{k}^{2}}{Q_{\infty}^{2}}
$$

The contribution of that surface element to the total aerodynamic load is then,

$$
\Delta \mathbf{F}_{k}=-C_{p k}\left(\frac{1}{2} \rho Q_{\infty}^{2}\right) \Delta S_{k} \mathbf{n} k
$$

where $\rho$ is the fluid density and $\Delta S_{k}$ is the area of the $k^{t h}$ panel. The total aerodynamic load, comprised of the contributions from all panels, can then be used to determine directional lift loads. 


\section{Chapter 5}

\section{Flight Test Preparation}

To obtain the experimental airspeed and pitch data to implement the Panel Method described in the previous chapter, flight tests were conducted using the Diamond DA20-A1

Katana. Research being carried out by Dr. Langlois' team at Carleton University, consisting of Jonathan Plumpton and Suzanne Swaine, also required flight testing. The objectives and efforts of both research projects were combined into a single flight testing program and with the cooperation of Ottawa Aviation Services, flight data was recorded using installed instrumentation as the aircraft performed a variety of manoeuvres outlined in the flight test plan that was developed. The creation of the flight test plan, a description of the developed flight data acquisition system and its installation, the various operational tests of the system, along with the process undertaken to obtain a flight permit are presented in this chapter.

\subsection{Flight Test Plan}

The flight test plan was composed to represent the flight conditions that a Katana aircraft at OAS would experience. As the fleet of Katana aircraft at OAS are used for flight training purposes (as opposed to being used for ferry flights or transportation/cargo), an initial list of flight manoeuvres was compiled by consulting the Transport Canada Flight Training Manual, [25]. Additionally, the Standard Operating Procedures used by OAS, the Diamond Katana Pilot Operating Handbook, and consultations with the Chief Flight Instructor at 
OAS were all used to compose the flight test plan. The flight test was also being used by Dr. Langlois and his research team at Carleton University to acquire flight data, which required a number of additional flight manouevres. The testing was initially split into three 2-hour flights (see Table 5.1) to alleviate pilot fatigue and to accommodate battery limitations imposed on the installed instrumentation. However, this was later modified to include all manoueuvres within two flights. The full flight test plan can be found in [26].

Table 5.1: Manouevres used in the flight test.

\begin{tabular}{|c|c|c|c|c|c|}
\hline \multicolumn{2}{|r|}{ Flight 1} & \multicolumn{2}{|r|}{ Flight 2} & \multicolumn{2}{|r|}{ Flight 3} \\
\hline $\begin{array}{l}\text { Time } \\
(\text { mins })\end{array}$ & Task & $\begin{array}{l}\text { Time } \\
(\text { mins })\end{array}$ & Task & $\begin{array}{l}\text { Time } \\
(\text { mins })\end{array}$ & Task \\
\hline 10 & Start \& Take-off & 10 & Start \& Take-off & 10 & Start \& Take-off \\
\hline 10 & Transit & 10 & Transit & 10 & Transit \\
\hline 5 & $\begin{array}{l}\text { High Altitude } \\
\text { Level }\end{array}$ & 5 & $\begin{array}{c}\text { Low Altitude } \\
\text { Level }\end{array}$ & 3 & Flight Stability \\
\hline 12 & Phugoid Motion & 10 & Climb & 12 & Phugoid Motion \\
\hline 3 & Flight Stability & 3 & Flight Stability & 6 & $\begin{array}{l}\text { Short Period } \\
\text { Motion }\end{array}$ \\
\hline 6 & $\begin{array}{c}\text { Short Period } \\
\text { Motion }\end{array}$ & 12 & Phugoid Motion & 5 & Side Slips \\
\hline 10 & Stall & 6 & $\begin{array}{c}\text { Short Period } \\
\text { Motion }\end{array}$ & 25 & Circuits (CYRP) \\
\hline 5 & Spin & 5 & Turns & 10 & Transit \\
\hline 5 & Slow Flight & 5 & Side Slips & 5 & Landing \\
\hline 7 & Spiral Dive & 10 & Transit & & \\
\hline 5 & Side Slips & 5 & Landing & & \\
\hline 10 & Transit & & & & \\
\hline 5 & Landing & & & & \\
\hline 93 & Total & 81 & Total & 86 & Total \\
\hline
\end{tabular}




\subsection{Flight Data Acquisition and Instrumentation}

As a light utility aircraft, the DA20-A1 Katana does not have a native flight data instrumentation package common in aircraft used for flight test purposes. As such, an aftermarket package had to be created and installed on the aircraft. The Katana was the focus of an additional research project being undertaken by Dr. Langlois at Carleton University which also required a flight data acquisition system and flight testing. The requirements of both project groups were combined to develop a single flight data acquisition system to be used during flight tests. The specific components of this larger flight data system, namely the ArduPilot Mega and differential pressure sensor, pertinent to aerodynamic load determination are covered here.

\subsubsection{ArduPilot Mega}

The open-source electronics prototyping platform, Arduino, was initially considered to create the instrumentation package due to its flexible and easy-to-use hardware and software. This allows the package to be cost effective and easily accessible and reproduced. The ArduPilot Mega (APM) (Figure 5.1), an Arduino-based amateur UAV autopilot system, was chosen as the central flight data recording system due to its compact size and multitude of on-board sensors (see Table 5.2).

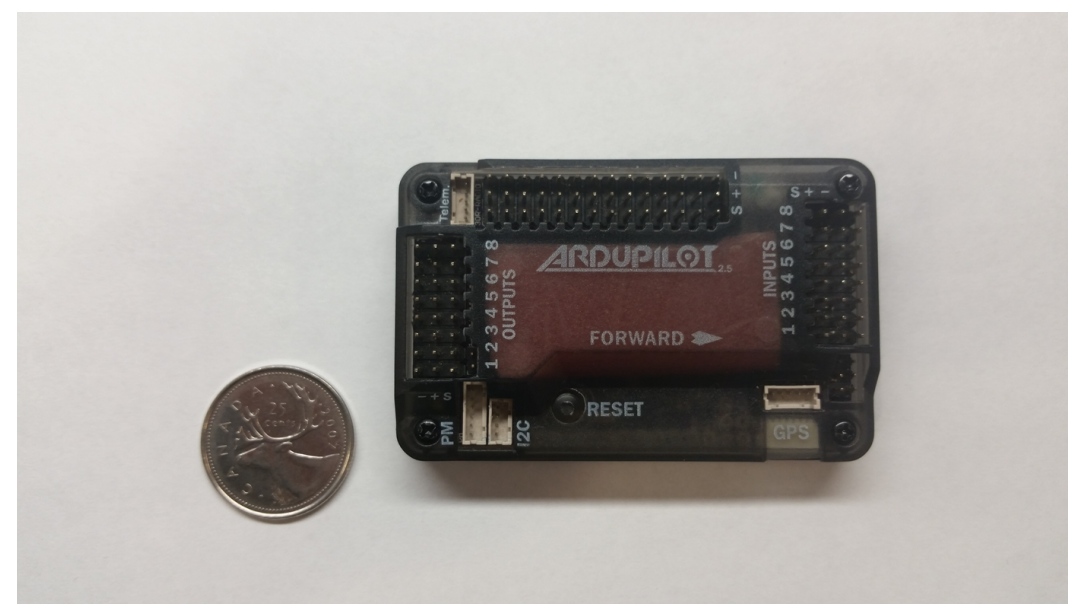

Figure 5.1: ArduPilot Mega (APM) used to centralize flight data acquisition. 
Table 5.2: ArduPilot onboard sensors

\begin{tabular}{|c|c|c|c|c|}
\hline Component & Manufacturer & Part Number & Resolution & Unit \\
\hline $\begin{array}{c}\text { 6-axis (rate gyroscope } \\
\text { +accelerometer) IMU }\end{array}$ & InvenSense & MPU-6000 & $0.2[27](80)[27]$ & $\circ / \mathrm{s}(\mathrm{mg})$ \\
\hline Barometer & $\begin{array}{c}\text { Measurement } \\
\text { Specialties }\end{array}$ & MS5611-01BA03 & $0.1[28]$ & $\mathrm{m}$ \\
\hline Digital Compass & Honeywell & HMC5883L-TR & $1-2[29]$ & degrees \\
\hline GPS & Mediatek & MT3329 & $3[30](0.1)[30]$ & $\mathrm{m}(\mathrm{m} / \mathrm{s})$ \\
\hline Pressure & Freescale & MPXV7002 & $0.01[31]$ & $\mathrm{kPa}$ \\
Sensor (Airspeed) & Semiconductor & & N/A & $\mathrm{N} / \mathrm{A}$ \\
\hline Processing Chip & Atmel & ATMEGA2560 \& & & \\
\hline
\end{tabular}

The APM is commonly used to provide a description of UAV dynamics in real-time at a sampling rate of approximately $30 \mathrm{~Hz}$. The inertial measuring unit (IMU), containing a 3axis gyroscope and 3-axis accelerometer, provides angular rate and linear acceleration data which is collectively used to calculate attitude. The on-board 3-axis magnetometer (digital compass) and connected GPS unit provide orientation and location information to produce bearing and track data. Additionally, the on-board barometer provides temperaturecompensated altitude data. Finally, an accompanying differential pressure sensor can be attached to determine airspeed.

As was discussed in Chapters 3 and 4, angle of attack and airspeed values are needed to determine the aerodynamic loads. This information is provided by the APM's IMU and attached differential pressure sensor respectively. The APM provided a means of acquiring these two pieces of information and eliminated the need to procure individual components, connect them, calibrate them, and store data.

\subsubsection{GoPro Video Camera}

In addition to the APM, a GoPro Hero video camera was included as part of the flight test setup (Figure 5.2). It records video footage at $30 \mathrm{~Hz}$ and $720 \times 640$ pixel resolution. Initially, 
this camera was added solely as a means of visually documenting the testing procedures on the day of flights and was not intended to be used for any data acquisition. However, this camera would eventually become a very important component in obtaining airspeed information as the differential pressure sensor malfunctioned, as explained in Chapter 6 .

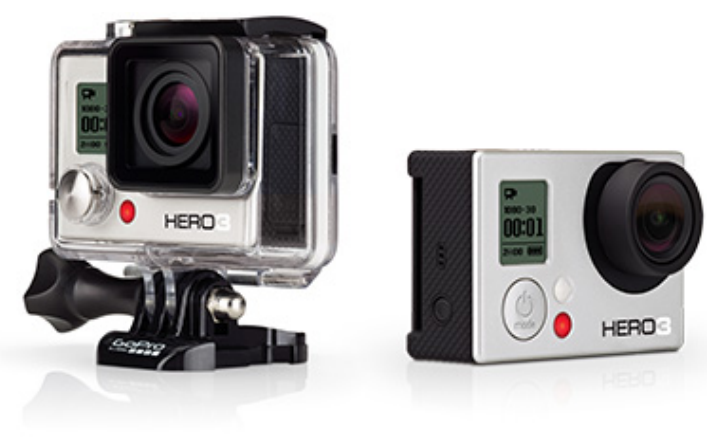

Figure 5.2: GoPro video camera. [32]

\subsection{Equipment Installation}

As the Diamond Katana is a light utility aircraft, space within the cockpit is very limited and all the equipment used during the flight test had to be located in the rear baggage area. A wooden platform was securely fastened within the baggage compartment and all other equipment was attached to this platform, including the APM. The GoPro camera was mounted using a stand which provided extra elevation for a useful view through the windshield of the aircraft. This installation of equipment is shown in Figure 5.3.

\subsubsection{Airspeed Pressure Sensor}

An accurate means of determining airspeed in flight was sought and many methods were explored. Ultimately, it was decided to tie into the Diamond Katana's native pitot-static pressure system, which is used to provide the pilot with altimeter, vertical speed and airspeed information while in flight. This was accomplished by tee-ing the static and pitot lines running into the aircraft's airspeed indicator (Figure 5.4) to allow a connection to the 


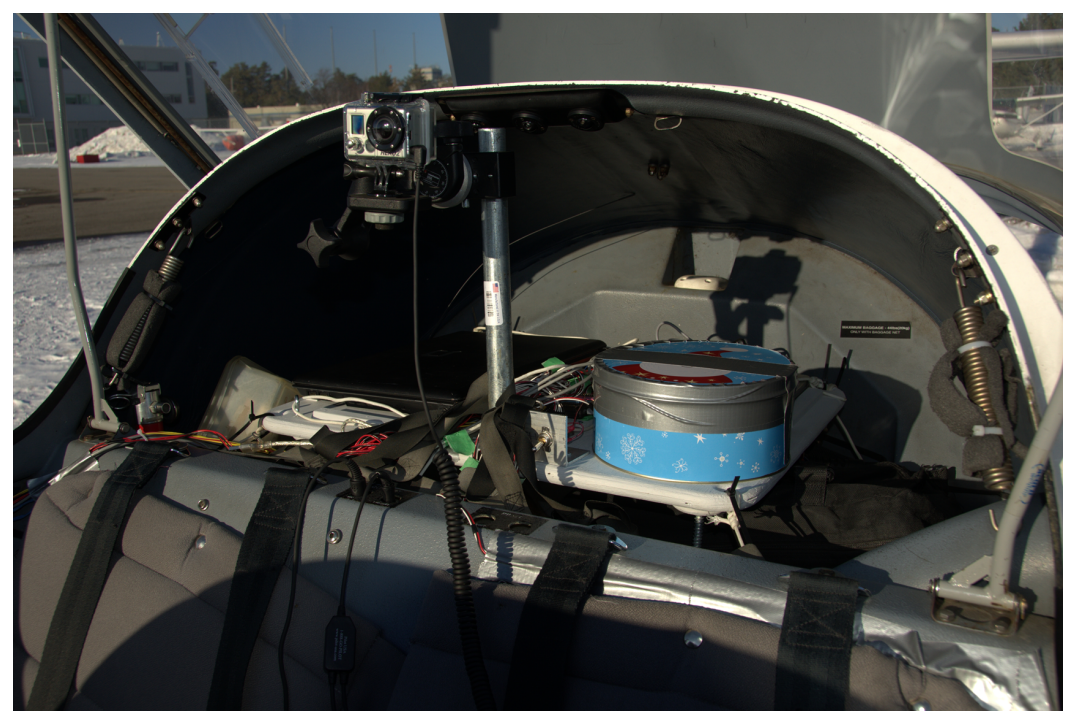

Figure 5.3: Installation of flight test equipment in the rear baggage compartment.

airspeed sensor (Figure 5.5) using a combination of nylon tube reducers. Due to a functioning pitot-static system being a regulatory requirement for all flights (Section 605.14 of [33]) a special flight permit had to be issued by Transport Canada, the governing body over all transportation in Canada. This flight permit is discussed in Section 5.5.

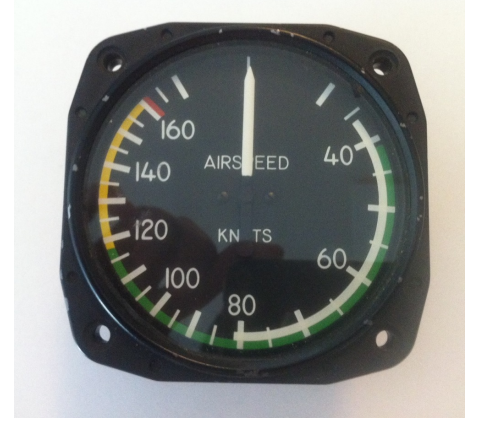

Figure 5.4: Diamond Katana airspeed indicator.

\subsection{Equipment and Instrumentation Testing}

Three tests were conducted prior to flight testing to ensure the reliability and operation of the entire flight data acquisition system. This includes a test of the differential pressure sensor used for airspeed determination, a low-temperature operational test of the entire data 


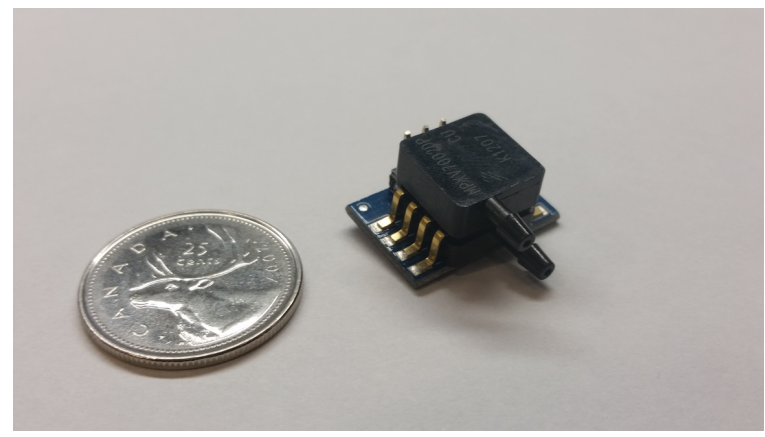

Figure 5.5: Differential pressure sensor installed for airspeed information.

acquisition system, and an operational test required by Transport Canada of the aircraft's pitot-static system.

\subsubsection{Pressure Sensor Test}

The differential pressure sensor is intended for use with amateur UAVs and its operational reliability at higher pressures (higher airspeeds) during flight testing of the Diamond Katana had to be verified. No manoeuvres were planned to be performed in excess of the aircraft's maximum manoeuvring speed of $104 \mathrm{kts}(53.5 \mathrm{~m} / \mathrm{s})$ [19], so a simple test to verify the operation of the pressure sensor up to this speed was performed.

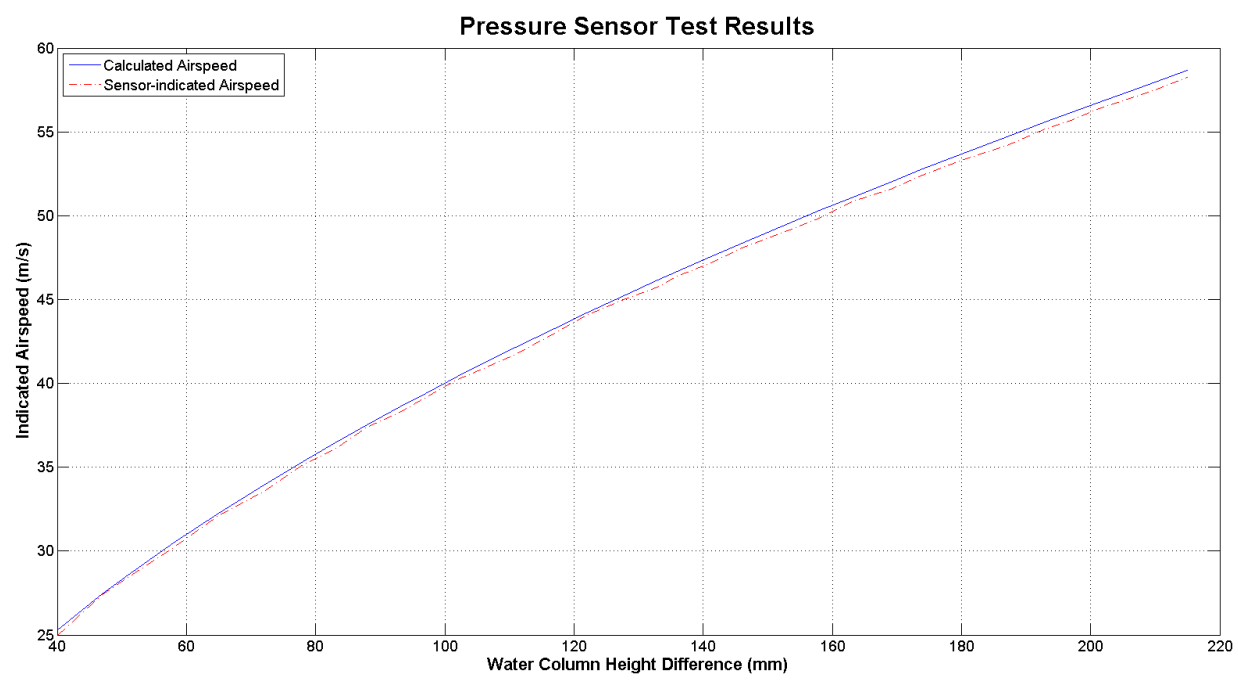

Figure 5.6: Pressure sensor test results 
In this test the static port of the pressure sensor was left open to atmosphere, while the dynamic port was connected to a water-filled U-tube manometer. Starting with equal heights of water on both sides of the manometer, water was incrementally added to the open end of the manometer. Every time additional water was added, the recorded pressure from the sensor was compared to the differential pressure as calculated from the manometer. These pressures were converted to airspeeds and are shown in Figure 5.6.

\subsubsection{Low-Temperature Operational Test}

With flight tests occurring during the winter months, the operation of the APM and the differential pressure sensor at low temperatures had to be ensured. The policy at Ottawa Aviation Services is to not allow any flights to take place when the ground temperature is below $-20^{\circ} \mathrm{C}$. As such, it was determined that the test should involve operation below $-21^{\circ} \mathrm{C}$ for a duration longer than the two hours expected for each flight. This test was conducted on a day where the outside air temperature was below $-21^{\circ} \mathrm{C}$ and the acquisition system was placed outdoors and left to run for 2.5 hours. The various sensors and equipment were triggered periodically throughout this test during which time all equipment continued to function properly. After the outdoor test, all equipment was brought indoors and continued to function properly. This eliminated any concerns surrounding condensation buildup.

\subsubsection{Pitot-Static System Test}

The greatest concern was that of the pressure sensor installation. Any leaks when tee-ing into the aircraft's pitot-static system would result in an unsafe situation for the pilot and passenger. A pressurization test was conducted by the Aircraft Maintenance Engineers at Ottawa Aviation Services prior to the flight tests. This pressurization test lasted approximately 8 hours, and would have to be performed before any further flights with the pressure sensor installed. This would be a costly time and resource penalty should the tests have to be repeated, in addition to a Flight Permit having to again be issued. This issue is revisited in Chapter 8. 


\subsection{Flight Permit}

While the inclusion of the APM system within the Katana cockpit is very unobtrusive, the alteration of the aircraft's pitot-static system (Section 5.3.1) and the installation of the other instruments comprising the larger flight data acquisition system (Section 5.2) resulted in the aircraft not conforming to the Certificate of Airworthiness originally issued by Transport Canada. As such, a Flight Permit (Specific Purpose) was sought under Section 507.4(3)(d) [33] and required a detailed Flight Test Plan (Section 5.1), description of all equipment installations and precautions taken to ensure safety during the flight.

A flight permit was ultimately obtained with the cooperation of a Transport Canada Design Approval Representative (DAR) who volunteered his services to Carleton University. Without this help the costs associated in obtaining a flight permit would be significant, and could very well have prevented the flight tests from taking place. This issue is revisited in Chapter 8. A copy of the acquired Flight Permit is included in Appendix A.

\subsection{Equipment Cost}

The work presented here in experimentally determining the aerodynamic loads on a wing spar is specific to a single flight on-board a particular aircraft. The ultimate goal of the research project is to produce a package that can be easily reproduced and installed on a variety of aircraft with minimal effort and cost. As mentioned earlier, the APM was used to acquire angle of attack information as it is very compact in size and eliminated effort to procure and connect sensors. The other vital piece of information needed for aerodynamic load determination is airspeed data. Discussed later in Chapter 6, is the malfunction of the pressure sensor intended to be used to obtain this airspeed data. As such, it will be shown that this information was eventually obtained using video footage recorded by the installed GoPro camera. Both these pieces of equipment, the APM and the GoPro camera, comprise the sole components needed to approximate aerodynamic loads over the course of a flight. Furthermore, the time, effort and resources needed to acquire a flight permit for installation of a pressure sensor (in order to acquire airspeed data) is completely avoided with the 
image processing method outlined in Chapter 6. Table 5.3 lists the approximate costs of the equipment used in the method presented shortly for aerodynamic load determination, which is less than $\$ 500$.

Table 5.3: Approximate cost of equipment needed for aerodynamic load determination.

\begin{tabular}{|c|c|}
\hline Component & Cost \\
\hline ArduPilot Mega & $\$ 250 \mathrm{CAD}$ \\
\hline GoPro camera & $\$ 200 \mathrm{CAD}$ \\
\hline Total & $\$ 450 \mathrm{CAD}$ \\
\hline
\end{tabular}




\section{Chapter 6}

\section{Airspeed Determination Through Video Processing}

The post-flight analysis of the flight test data revealed a malfunction of the differential pressure sensor resulting in erroneous airspeed information for the entire flight test. The time required to conduct the operational tests of the equipment, schedule a flight with a pilot, and repeat the flight tests would have extended beyond the expiration date of the flight permit described in Section 5.5. As such, the work then focused on the video footage from the camera mounted within the cockpit as it provided a view of the instrument panel including the airspeed indicator (outlined in red in Figure 6.1). With the video footage containing the only recorded airspeed information, a video processing method was developed to obtain the required airspeed data. Provided in this chapter is the theory related to image/video processing and an explanation of the developed method with examples of the results at each step of the process.

\subsection{Video Footage and Image Processing}

A GoPro video camera was mounted in the baggage compartment of the Katana's cockpit solely as a means of documenting the flight testing that was to take place. As such, it was positioned to provide a view out of the aircraft's windshield similar to the pilot/passenger perspective. However, this placement also provided a view of the entire instrument panel as 


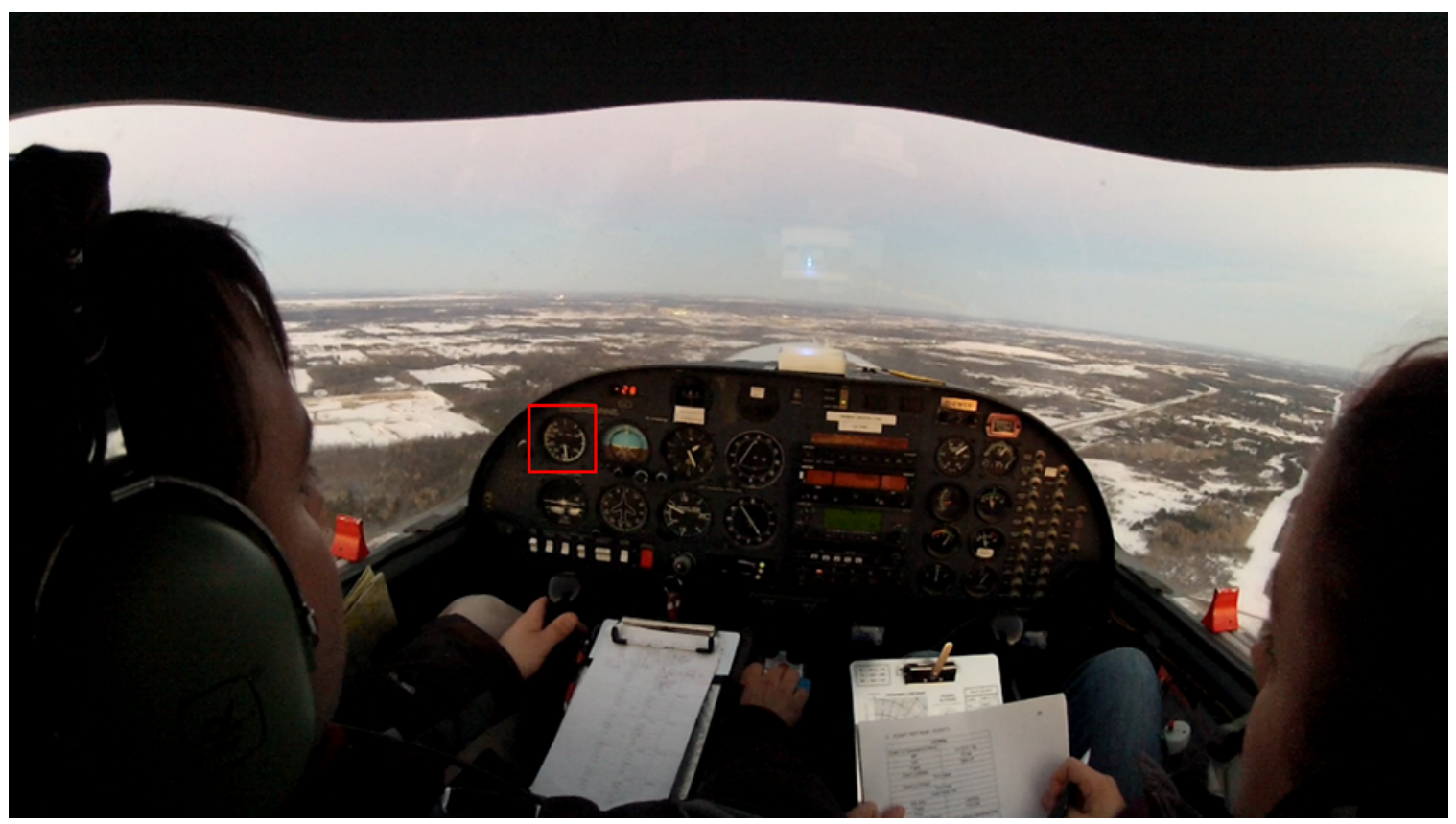

Figure 6.1: GoPro camera view of the Katana's instrument panel with the airspeed indicator outlined in red.

shown in Figure 6.1. The video footage was captured at $30 \mathrm{~Hz}$ and $720 \times 640$ pixel resolution.

A video is a collection of static images or frames which may or may not have associated audio data. When frames are extracted from a video sequence they can be processed in a similar manner to which an image is processed and all image processing operations can be performed on an extracted frame [34].

In order to obtain the airspeed information, the needle of the airspeed indicator (ASI) needed to be isolated from the rest of the image. The first step in isolating the needle was to extract individual images from the video footage. Although the footage was recorded at $30 \mathrm{~Hz}$ only every third image was extracted (sampling rate of $10 \mathrm{~Hz}$ ) to expedite the capturing process and to limit the quantity of images requiring processing. The simplest way to isolate the needle from the rest of the full-size image was to crop the region of interest. The widely used Portable Network Graphic (.png) image format is a lossless compressed format [35] that allowed a large number of images to be stored while preserving the limited image quality (from the already lossy .mp4 video) for future processing. An example of the cropped image is shown in Figure 6.2(a). 


\subsection{Image Processing and Object Detection in MATLAB}

Object detection in videos involves verifying the presence of an object in image sequences and in some cases possibly locating it precisely for recognition [34]. Objects are characterized by their salient features such as colour, shape, texture or any other features [36]. There exists many methods to determine whether or not an image contains a defined object; however, this first step was simplified due to the fact that the airspeed indicator (ASI) and its needle were known to be contained in the image and the problem then becomes indicating its position.

When processing a video sequence (successive images) a tracking method can be implemented whereby the object cannot only be tracked but its position (and sometimes size) followed as seen in the succession of images [36]. This "object tracking" was not implemented due to the very minor changes in position and size of the needle from frame to frame.

MATLAB R2013a was used for all the image processing described here as it includes an extensive list of built-in functions. The use of these functions in isolating the ASI needle from the cropped images are explained in the following subsections.

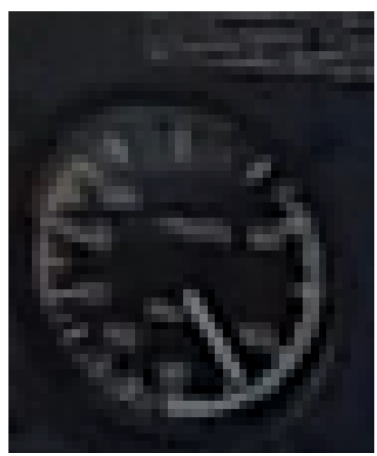

(a)

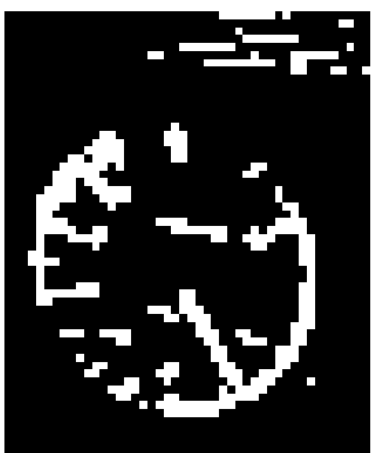

(b)

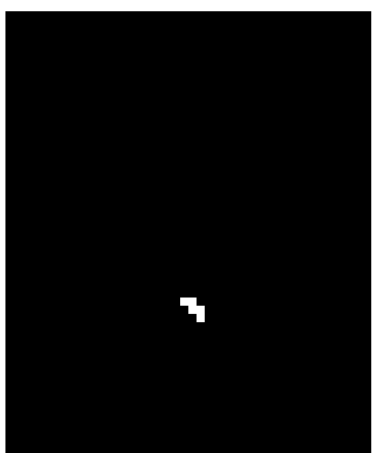

(c)

Figure 6.2: Processing images (a) cropped image (b) binary image (c) masked binary image showing only the ASI needle. 


\subsubsection{Binary Image}

Objects can be separated from an image based exclusively on their colour [36]. As can be seen in Figure 6.2(a), the ASI is comprised of a white needle on a black background and this method of object detection was implemented. This black and white colouring allowed all other colours in the cropped image to be disregarded, and permitted the use of black and white images (binary images) when detecting the ASI needle. As such, MATLAB's im2bw( $\mathrm{I}$, level) built-in function was used to convert the RGB colour image, I, to a binary image (Figure 6.2(b)). This is carried out by first converting the colour image to a greyscale image, after which each pixel in image $I$ is compared to a specified luminance value, level, which ranges from $0-1$. All pixels with luminance greater than the specified level value are given a value of 1 (white) with all other pixels given a value of 0 (black).

The choice of a luminance value is arbitrary and posed a problem when trying to create a set of binary images. This problem is most easily depicted in Figure 6.3, which shows the large variation in brightness of four images from the flight test. The varying lighting conditions over the course of the flights changed due to the time of day, the sunlight being cast on the instrument panel, as well as glare due to the sunlight. This variation in lighting resulted in different contrasts for each video frame meaning that the specified level value would be unique for each image being processed. This problem was solved using an iterative approach to setting the level value, whereby it would be increased until a usable binary image was produced. This process is depicted in Figure 6.4, the components of which are further discussed in the following subsections. 

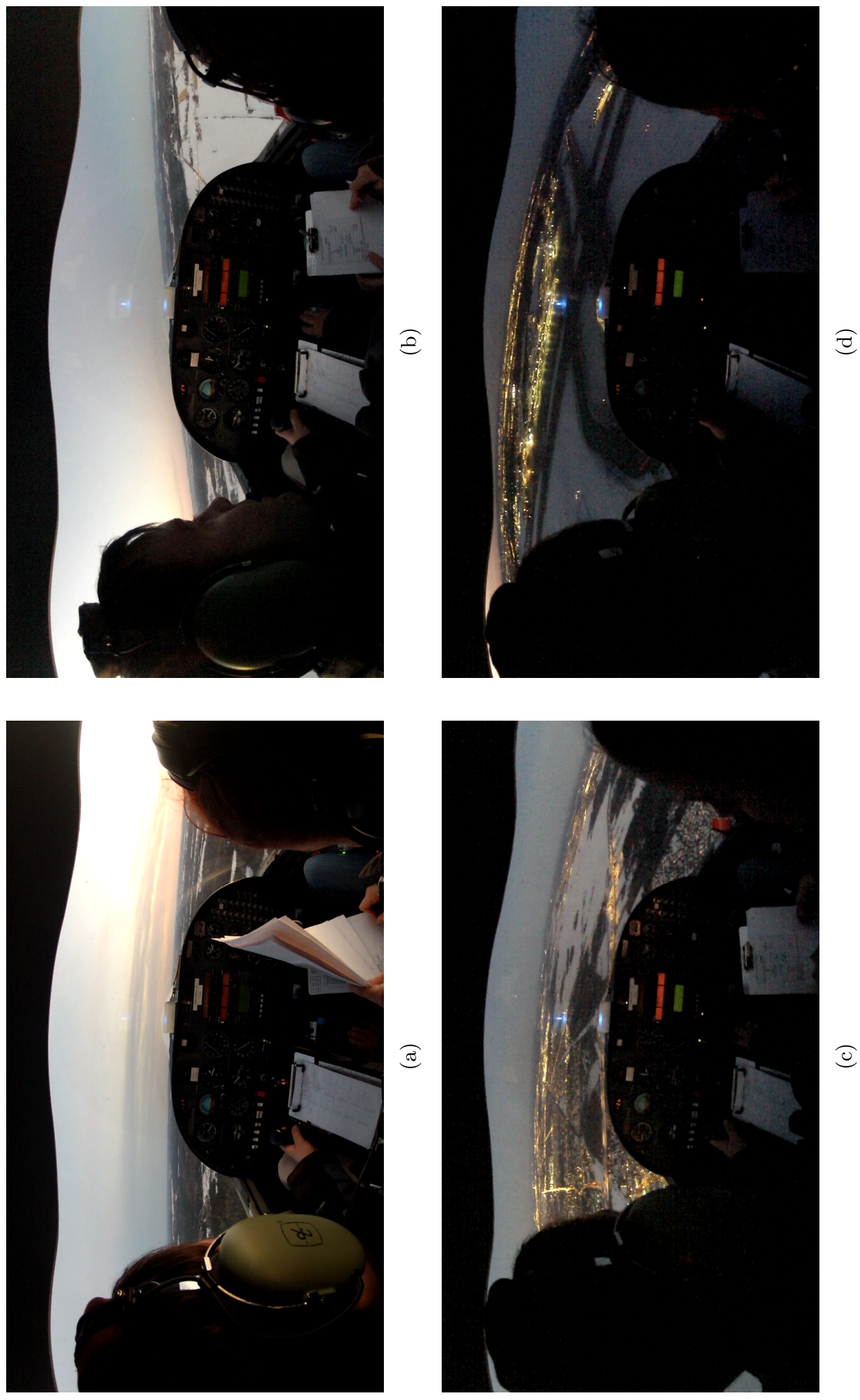

Figure 6.3: Luminance variation in cockpit footage. 


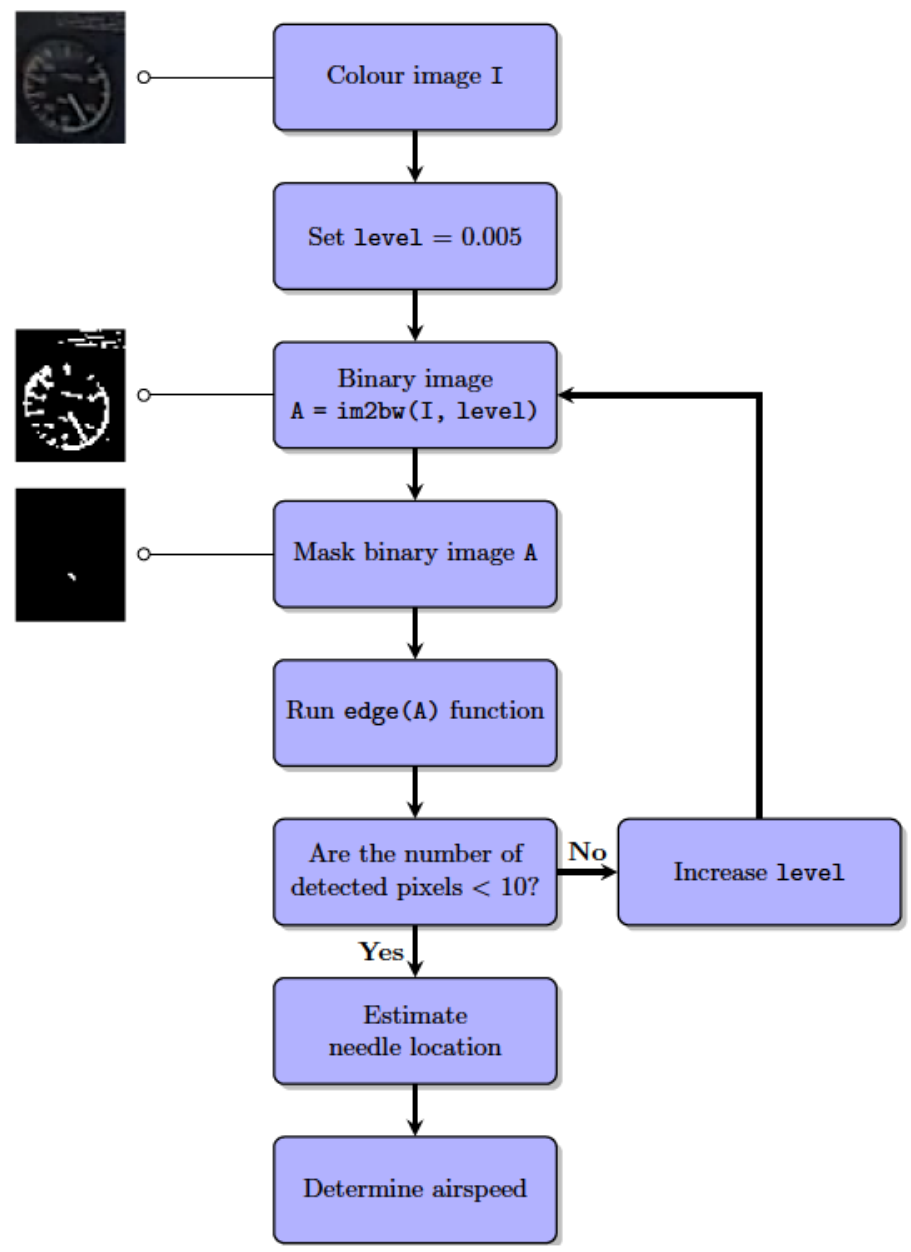

Figure 6.4: Image processing method for airspeed determination.

\subsubsection{Image Masking and Clutter}

With a video frame now cropped and converted to a binary image (Figure 6.2(b)), isolating the ASI needle could begin. Knowing that the needle is represented only by white pixels, all other white pixels not part of the ASI needle were made black (masked). MATLAB uses a (row,column) format to express a pixel location within an image. By approximating the pixel location of the centre of the ASI, all pixels outside a specified radial distance were masked. This radial distance was set to mask the circumference of the ASI including the white airspeed markings so that the only white pixels left in the image were those of the needle itself. This masking is portrayed in Figure 6.5 where the red sections represent areas 
of the ASI that were omitted from the binary image, leaving behind an area for the needle to be detected. An example of the final result of this masking is shown in Figure 6.2(c).

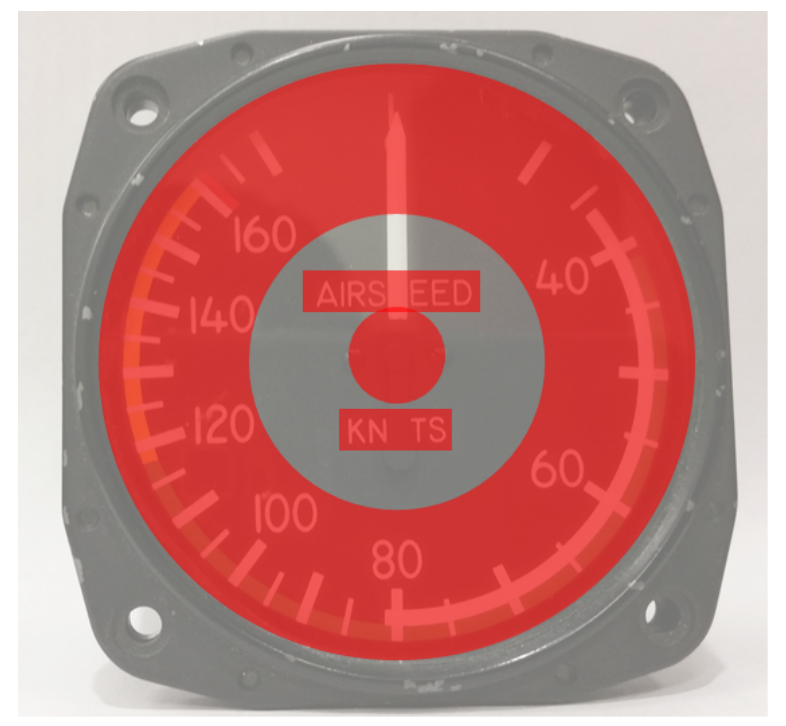

Figure 6.5: Depiction of the masked sections of the ASI to isolate the needle.

\subsubsection{Locating the Airspeed Indicator Needle}

With a binary image containing only white pixels of the ASI needle (Figure 6.2(c)) the location of the needle needed to be determined. MATLAB's edge(A) function finds the boundaries of objects within an image A by detecting discontinuities in brightness of pixels. The function returns a set of pixel locations that represent the boundary of the object, which in this case was the needle. With a completely black and white image this function produced very good results.

As mentioned in Section 6.2.1, an iterative process was used to set the level value to produce the binary image. Setting this value too low resulted in an image with many white pixels (Figure 6.6(a)), making it harder to discern the actual location of the needle. Setting this value too high produced too dark of an image and hid the needle (Figure 6.6(b)). The iterative process (increasing the level value) was repeated until MATLAB's edge(A) returned fewer than 10 pixels comprising the outline of the needle. This number of pixels was chosen based on trial and error to produce usable images. 


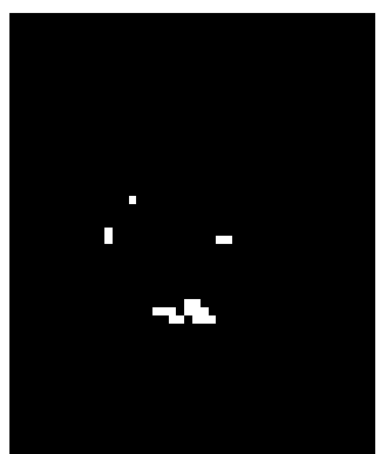

(a)

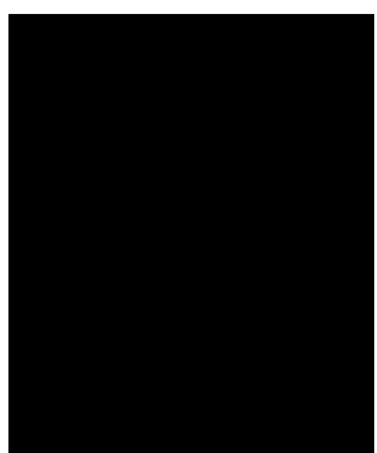

(b)

Figure 6.6: Examples of a binary image with a level value set too: (a) high (b) low.

\subsubsection{Determining Airspeed}

With a binary image now only containing white pixels representing the ASI needle, the set of pixel locations were averaged to approximate a single location of the needle. The results of this step are shown in Figure 6.7 where the approximated ASI centre is shown along with the outlining pixels of the needle and the determined needle location.

\section{Edge Detection Results}

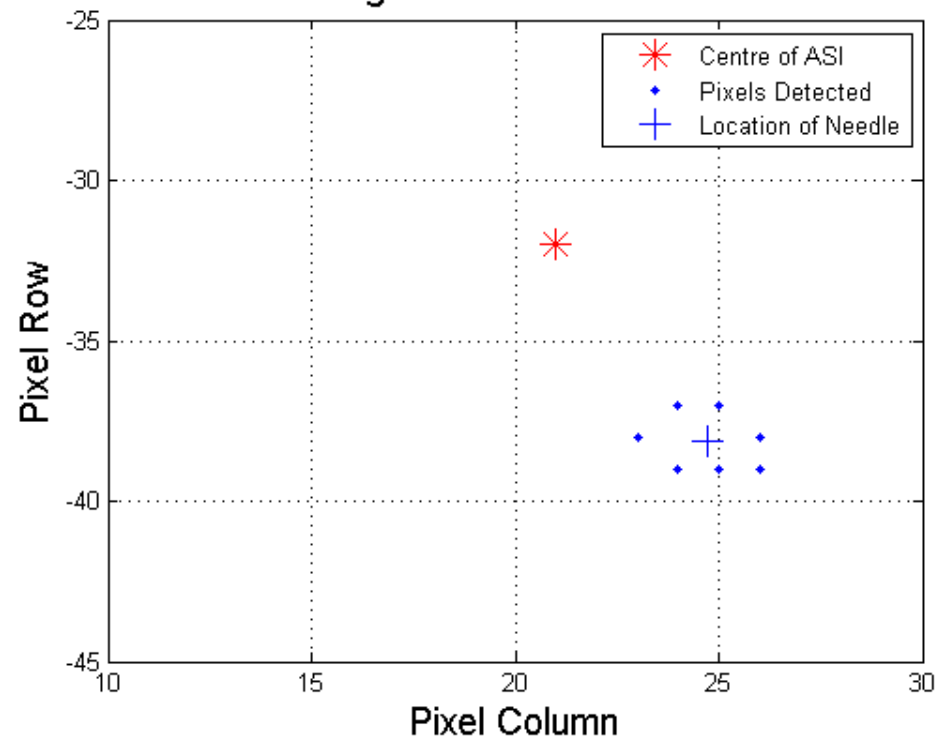

Figure 6.7: Results of MATLAB's edge(A) function to locate pixels and needle location.

Relating the determined needle location to an airspeed required a simple Cartesian-topolar coordinate conversion using the ASI centre (approximated earlier in Section 6.2.2) 
as the origin. This resulted in an angular value of the needle relative to the ASI centre. Similarly, pixel locations representing various airspeed markers were chosen, and associated marker angles were calculated. The chosen airspeed markers and their radials are depicted in Figure 6.8. The needle angle was finally used to linearly interpolate between the marker angles to determine the airspeed.

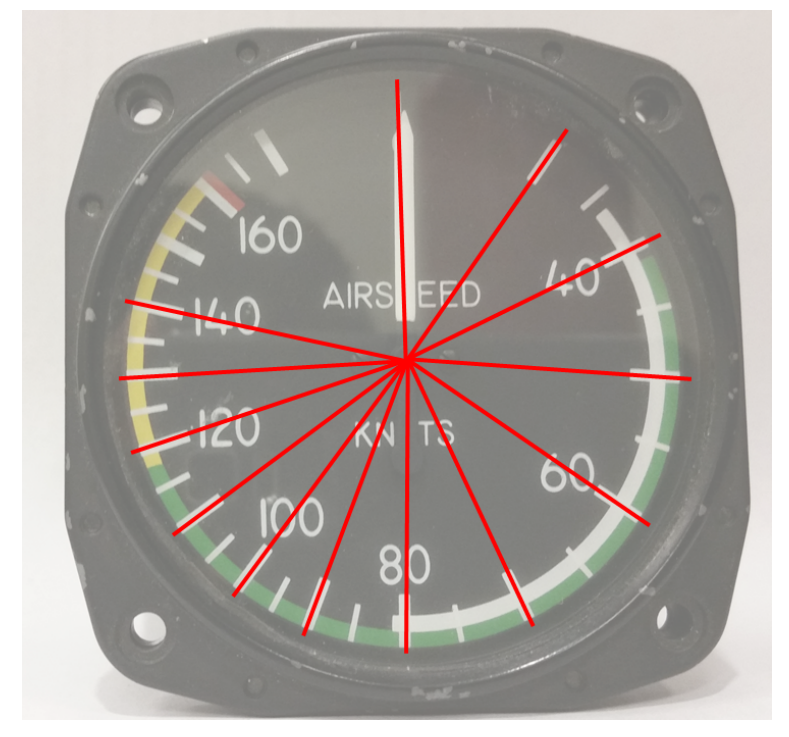

Figure 6.8: Airspeed markers used for interpolation.

\subsection{Results and Verification of the Developed Method}

This method of determining airspeed via image processing was verified using the KatanaSim developed by Dr. Langlois and his research team at Carleton University. The KatanaSim, shown in Figure 6.9, is a motion-based flight training simulator for the Diamond Katana which runs using X-Plane, a flight simulation software. The KatanaSim was built using an actual Katana cockpit section and includes commercially-available simulated instruments in the instrument panel. These instruments are similar in appearance to those used in the Katana aircraft. A simulated flight was carried out with X-Plane recording the airspeed information that was used to control the simulated ASI within the KatanaSim cockpit. A GoPro camera was also installed within the cockpit to capture video footage of the 
instrument panel, similar to the setup used in the initial flight tests. The recorded video footage was used with the described image processing method to obtain airspeed data.

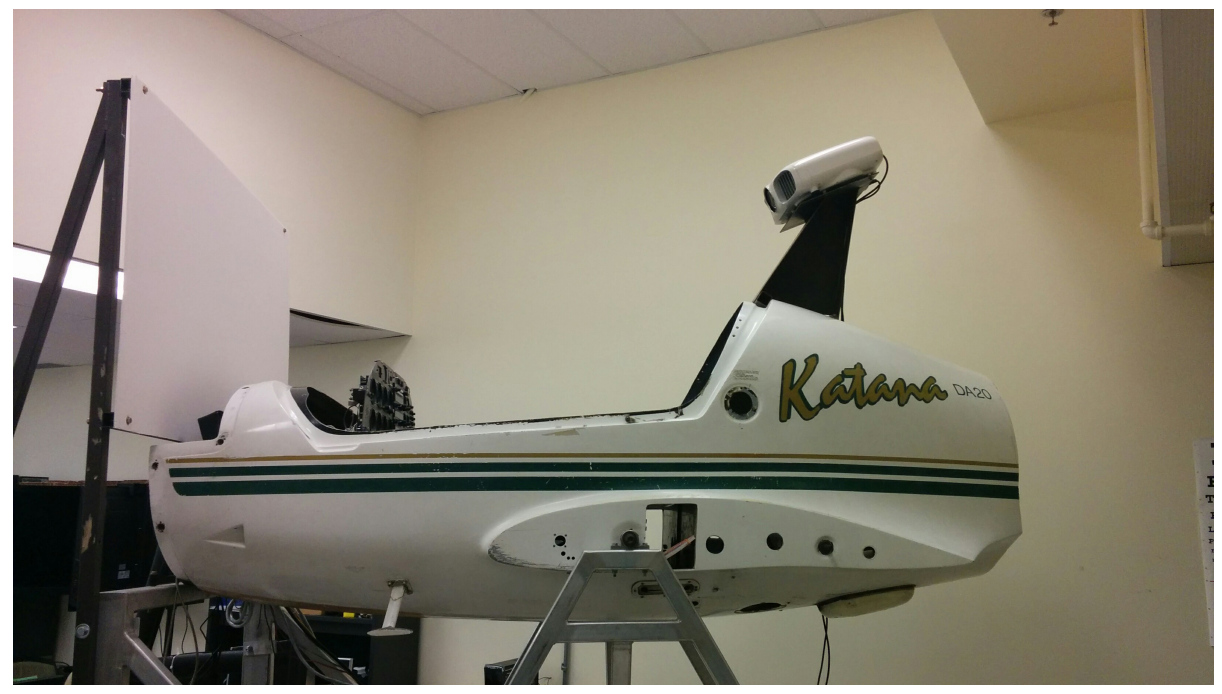

Figure 6.9: The KatanaSim motion-based flight training simulator.

This obtained airspeed information is compared with the airspeeds recorded by X-Plane in Figure 6.10. The areas of deviation occur at times of rapid change in speed. These deviations are thought to be an artifact of the simulator setup in that the simulated ASI in the cockpit is believed to lag the X-Plane outputs. All other portions of the test showed very close correlation of the method developed and shows promise as a means of obtaining airspeed information.

The main advantage to this method is the elimination of a differential pressure sensor that must be tied into the aircraft's native pitot-static system and the associated flight permits and approval from a transportation authority. Being able to obtain airspeed information solely from an installed camera within the cockpit has many applications due to its ease and unobtrusive setup. 


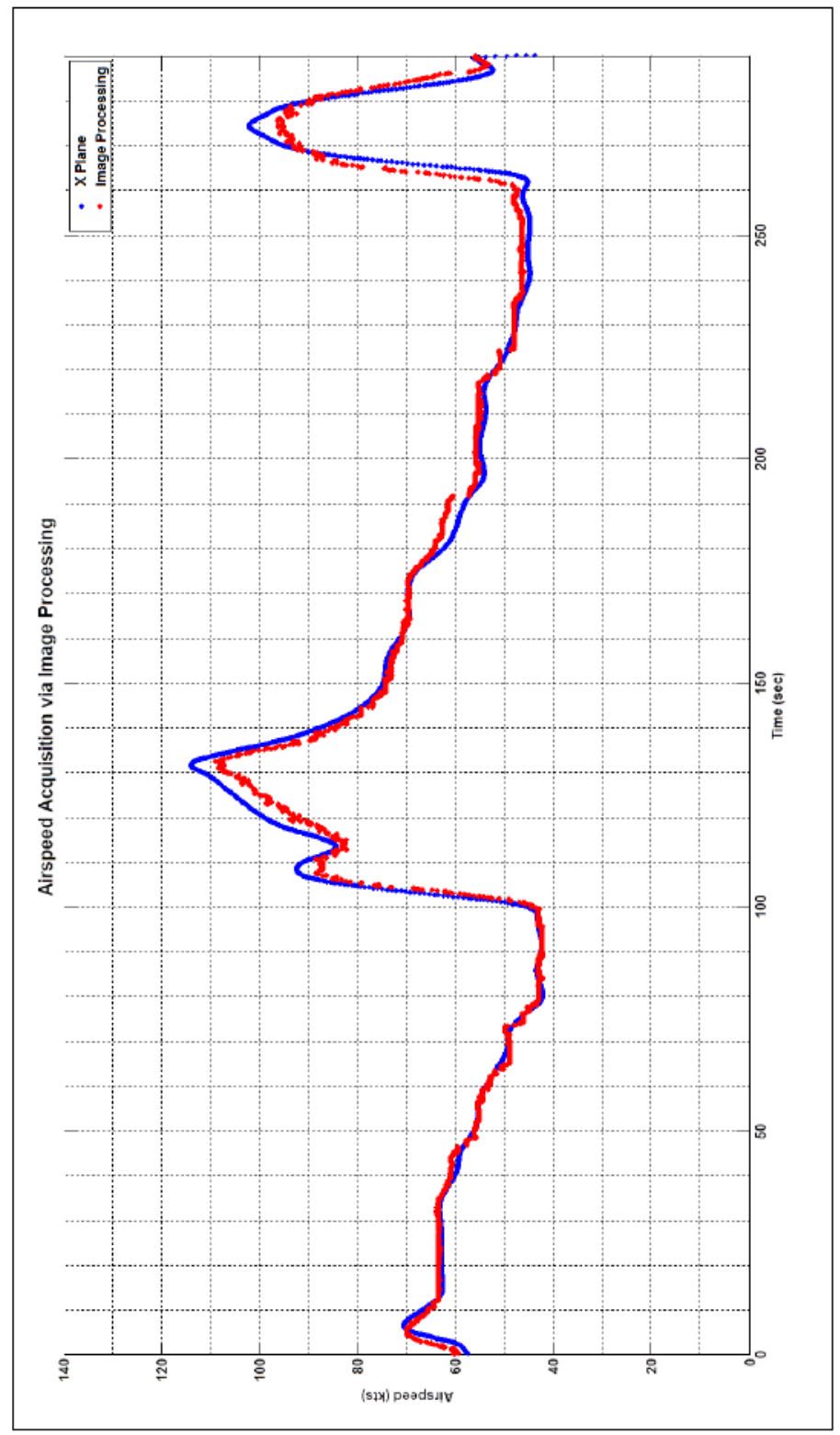

Figure 6.10: Airspeed obtained via image processing compared to airspeeds generated by X-Plane. 


\section{Chapter 7}

\section{Panel Method Implementation and Aerodynamic Load Determination}

This chapter combines the acquired airspeed using the image processing method of Chapter 6 with pitch data from the ArduPilot Mega (APM) to carry out the Panel Method theory of Chapter 4. Section 7.1 describes the verification of the developed Panel Method code, while Section 7.2 outlines the determination of the aerodynamic lift load using the acquired flight data for the Diamond Katana.

\subsection{Three-dimensional Steady State Panel Method and Ver- ification}

The developed panel method was verified using the symmetric NACA 0012 airfoil due to the extensive experimental wind tunnel data available for it. In particular, experimental

data in the $\operatorname{Re} \approx 2.0 \times 10^{6}$ was used for verification, which is representative of the high Reynolds number region in which the flight testing occurred. By comparing experimental $C_{l}$ vs. $\alpha$ data with results from the panel method, a confirmation of the method was obtained. The steps of verifying the method is covered in this section.

As discussed in Section 4.3, the first step is the discretization of the 3-D airfoil. A $10 \mathrm{~m}$ span was used for verification to remain consistent with the span of the Diamond Katana. This discretization is depicted in Figure 7.1, where the nodes (panel corner points) and the 


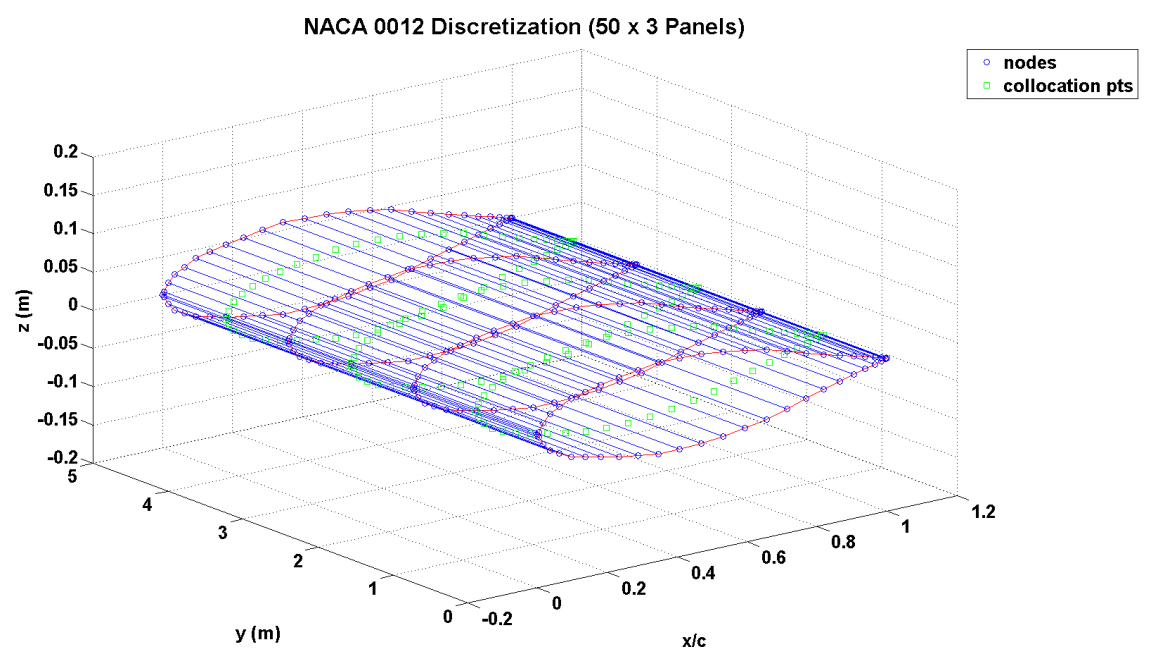

Figure 7.1: NACA 0012 discretization using the "full-cosine" method.

collocation points (in green) are shown for a semi-span section. Three span-wise and 50 chord-wise panels were used, the selection of which is discussed in the following subsection.

\subsubsection{Coefficient of Pressure Distribution and Coefficient of Lift Calcu- lation}

The coefficient of pressure, $C_{p}$, distribution was obtained for the NACA 0012 airfoil, an example of which is shown in Figure 7.2 for $\alpha=5^{\circ}$. Here, one can see the reduction in $C_{p}$ along the span of the wing which is consistent with a 3-D airfoil. Figure 7.2 also illustrates a limitation of the panel method which is that of a discontinuity at the leading edge. This is a result of the abrupt change in geometry in this region, combined with the finer discretization due to the use of the "full-cosine" method, which yields large changes in calculated local velocities.

The experimental airfoil data available is representative of an infinite $3-\mathrm{D}$ wing or a $2-\mathrm{D}$ airfoil where span-wise effects are neglected. As such, the obtained 3-D pressure distribution of Figure 7.2 was used to determine the total lift on the airfoil and averaged over the three span-wise panels. This 2-D lift value was then used to calculate the respective lift coefficient, $C_{l}$. 


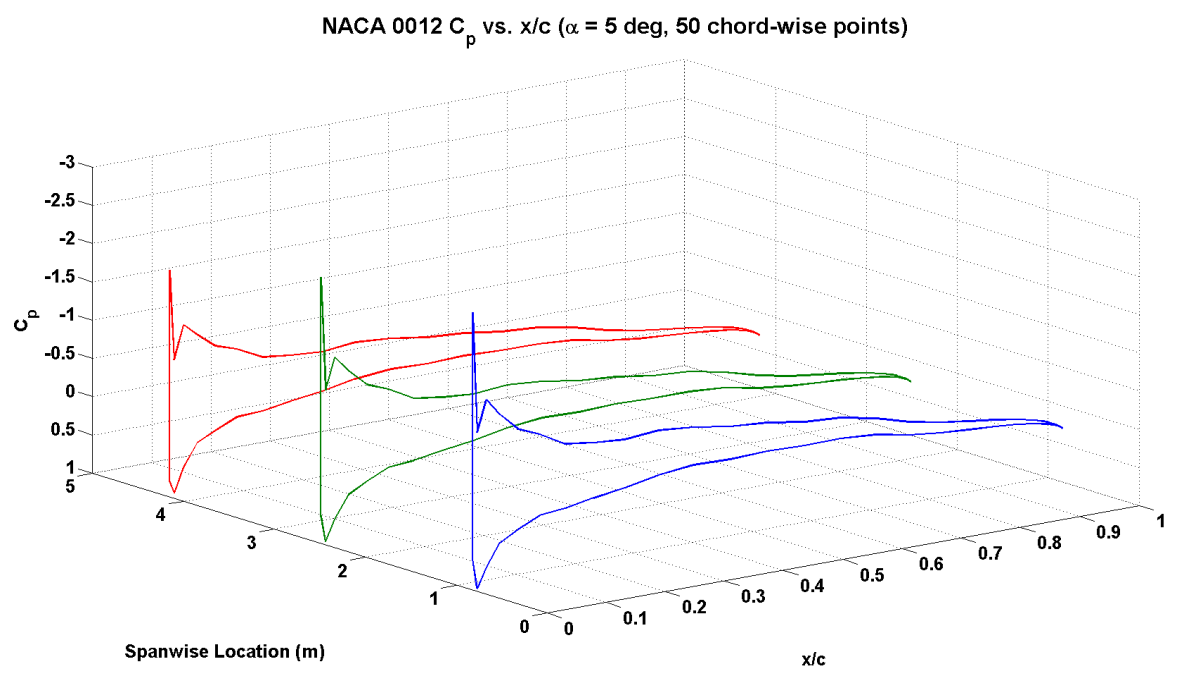

Figure 7.2: 3 -D $C_{p}$ distribution of the NACA 0012 airfoil at $\alpha=5^{\circ}$.

A convergence of the lift coefficient dictated the number of chord-wise panels that were used in the verification process. Figure 7.3 shows the convergence process where a $2 \%$ convergence was attained using 50 chord-wise panels.

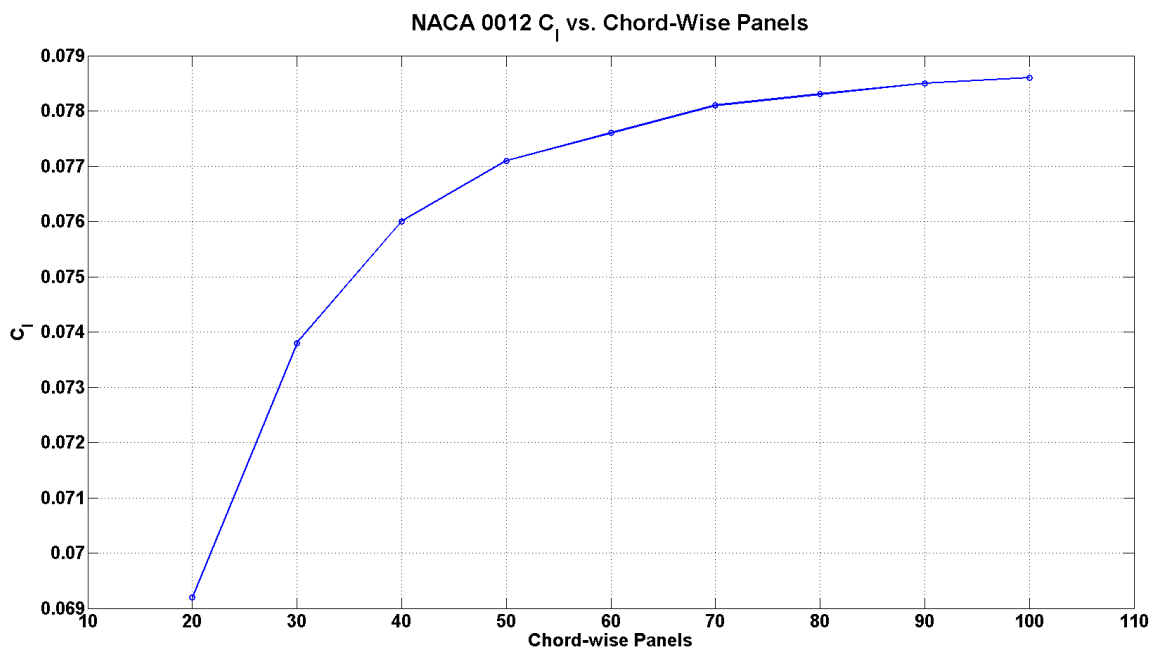

Figure 7.3: Lift coefficient convergence via chord-wise panels.

Having determined the number of panels to use, lift coefficients were determined over a range of $\alpha$, the results of which are shown in Figure 7.4. Found in [37] is a critical assessment of the NACA 0012 airfoil where a large body of experimental results were obtained using 
more than 40 wind tunnels. This assessment considered possible sources of error as a result of each facility while also considering wide ranges of Mach number, Reynolds number, and angle of attack. Using the accumulated experimental data, correlations were able to be made. One of these relationships is a lift-curve slope as a function of Reynolds number:

$$
C_{l_{\alpha}}=0.1025+0.00485 \log \left(\operatorname{Re} / 10^{6}\right) \quad \text { per degree, } \quad 2 \times 10^{6}<\operatorname{Re}<2 \times 10^{7}
$$

This line is also added to Figure 7.4, showing a match to determined $C_{l}$ values using the developed panel method.

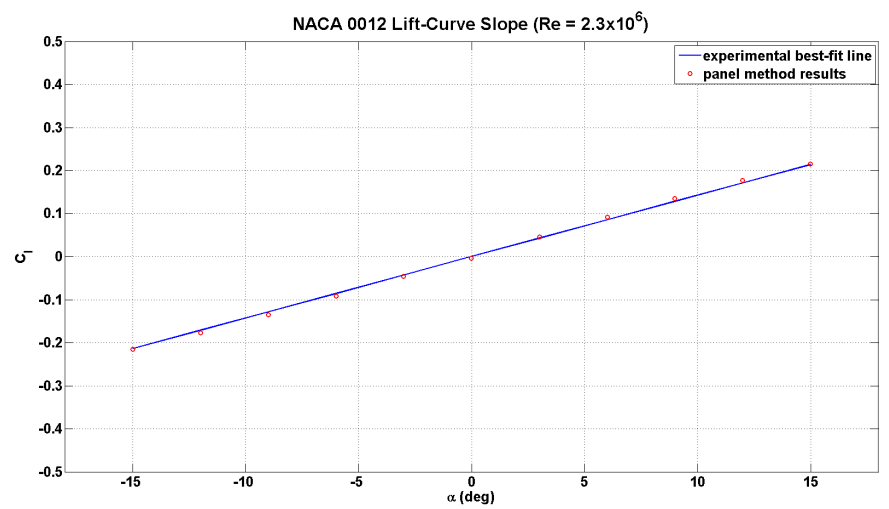

Figure 7.4: Experimental [37] and panel method NACA 0012 lift-curve slope.

\subsection{Aerodynamic Lifting Load Determination}

Having verified the lift-curve slope and the results of the developed panel method for a simple symmetric airfoil, the required aerodynamic lift loads for the Katana aircraft could be determined. This process is described in the following section.

\subsubsection{Discretization of the Wortmann FX 63-137 Airfoil}

The first step was to discretize the Katana's Wortmann FX 63-137 airfoil. Figure 7.5 depicts a semi-span section of the airfoil used, and although not to scale, it illustrates key parts of the discretization used. Figure 7.5(a) shows the nodes and collocation points used 
along with the "full-cosine" paneling method. A $1^{\circ}$ leading edge sweep was incorporated (Figure 7.5(b)) along with a $4^{\circ}$ dihedral (Figure 7.5(c)), aspects of which are found in the actual aircraft.
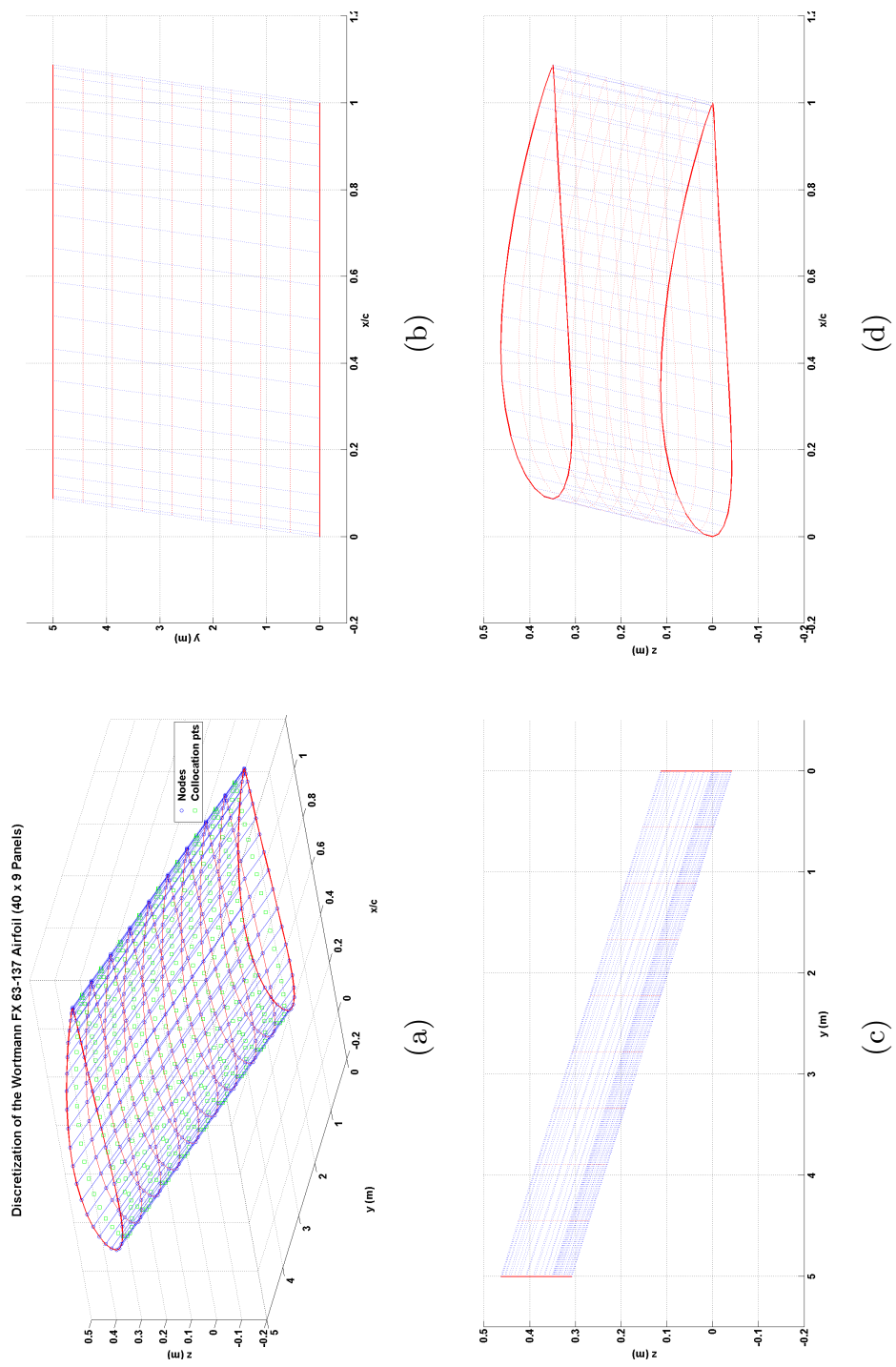

Figure 7.5: Semi-span discretization of the Wortmann FX 63-137 airfoil (not to scale). 
The number of panels to be used was chosen based on a convergence of the calculated coefficient of lift, $C_{l}$, based on a change in the number of chord-wise and span-wise panels used. The results of this process are shown in Figure 7.6. Ultimately, 40 chord-wise and 9 span-wise panels were selected as both these values provided a $2 \%$ convergence.
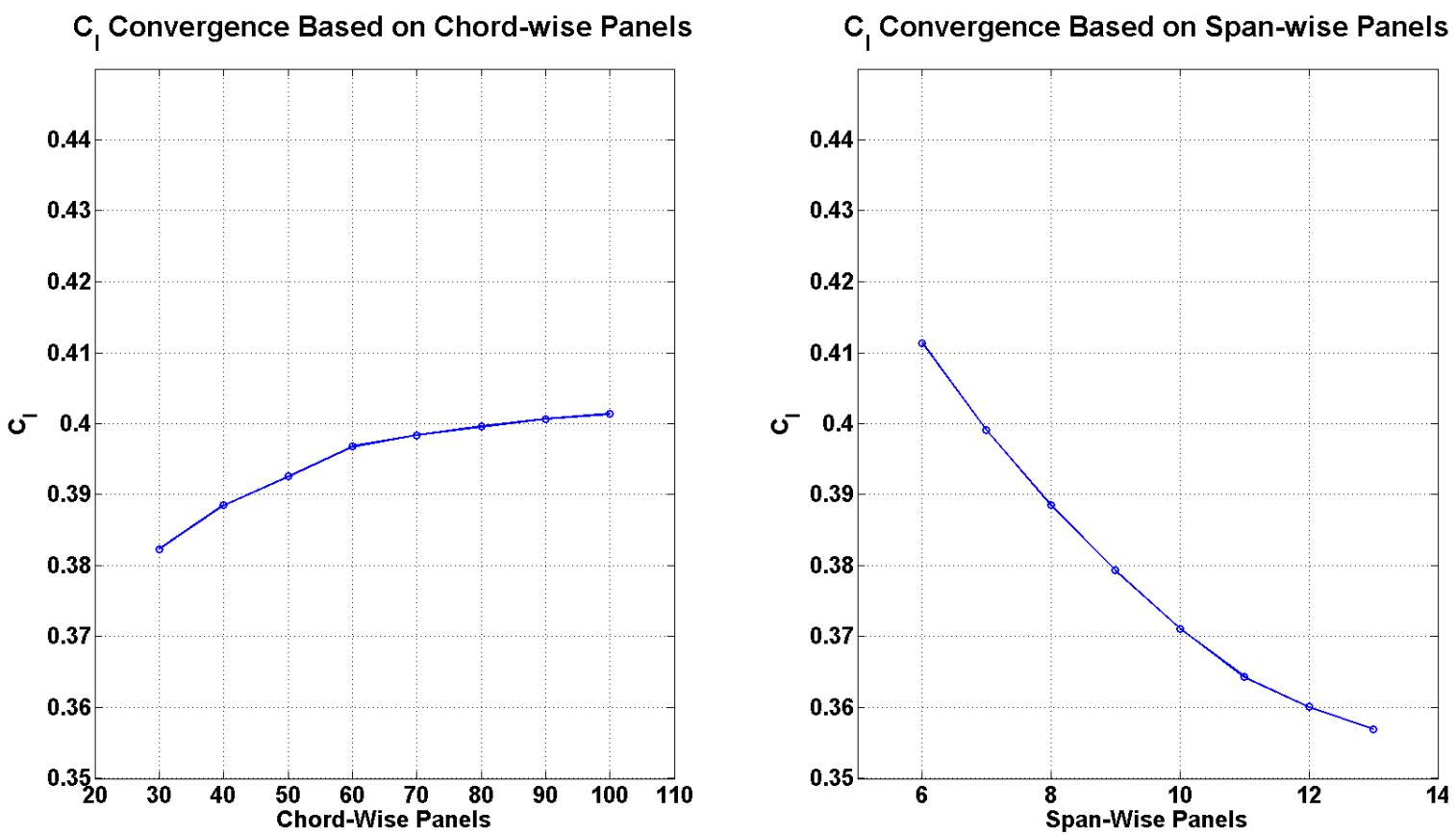

Figure 7.6: Determining the number of panels to use based on a $2 \%$ convergence of $C_{l}$.

\subsubsection{Aerodynamic Lift Loads}

With a discretized wing, the aerodynamic lifting loads could be calculated given aircraft pitch and airspeed. Pitch data was obtained using the on-board IMU in the ArduPilot, and airspeed data was obtained through the image processing outlined in Chapter 6 . With these three crucial pieces of information, the lifting load history was obtained for a segment of the flight test where the aircraft was flying wings-level with varying pitch and airspeed. The results of this segment are shown in Figure 7.7.

The beginning of this particular flight segment started with the aircraft flying straight and level, where the lift generated should have equaled the weight of the aircraft which was estimated to be $730 \mathrm{~kg}$ (approximately $7200 \mathrm{~N}$ ) from pre-flight weight and balance 

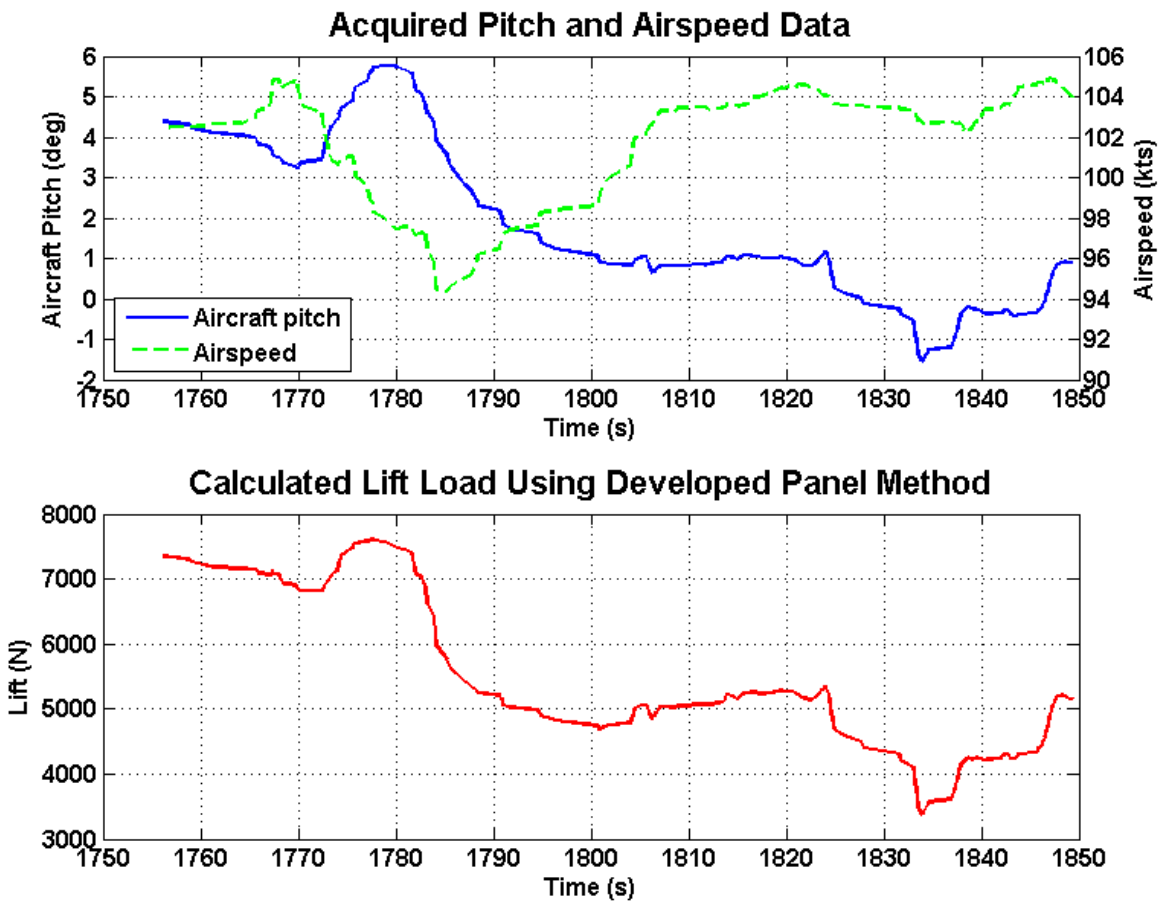

Figure 7.7: Aircraft pitch and airspeed data, and resulting calculated lift load.

documents. The results of Figure 7.7 show lift being produced in this range and provides validity to these results (in addition to the verification of the panel method outlined in Section 7.1). The remainder of this flight segment was a rapid descent in order to arrive at the airport before sunset. These results show the expected trend of decreased lift with decreased pitch. While airspeed was not varied as much as pitch during this flight segment, the increase in lift with an increase in airspeed (while maintaining constant pitch) can be observed from 1800-1820 seconds.

The two most obvious sources of error in this experimental determination of lift loads are the acquired pitch and airspeed data. The ArduPilot's on-board IMU provided pitch data with roughly $0.2^{\circ}$ of accuracy while the largest source of error is that of the airspeed. The airspeed indicator provides readings with an accuracy of \pm 2.5 knots. The image processing method used to obtain the airspeed used markers with 10 knot spacing to interpolate values. This limitation is addressed in the following Chapter, but the results presented would be sufficient to identify instances of extreme loading values in excess of predetermined limits. 


\section{Chapter 8}

\section{Conclusion}

The work presented in this thesis showed that a method of acquiring flight data from an in-service general aviation aircraft is possible. With cost being of concern, this was accomplished using amateur UAV components, namely the ArduPilot, whose compact design allowed it to be installed easily within the confines of a small cockpit. More importantly, a method of acquiring airspeed data from cockpit video footage was developed. The results of the image processing method described here showed very promising results. This process would permit the acquisition of this crucial piece of flight information in an extremely unobtrusive manner simply through the use of an installed camera. The greatest advantage of obtaining airspeed information in this way is the ability to avoid the lengthy and costly certification process required to tee into the aircraft's pitot-static system. Together, the ArduPilot and camera (along with the developed image processing method) provided aircraft pitch and airspeed information which was ultimately used to determine in-service aerodynamic lift loads experienced by the aircraft's wing spar. This load determination was accomplished using potential flow theory and a developed Panel Method.

The largest source of error introduced is a result of limitations on the airspeed indicator (ASI). At most, this instrument provides the pilot with \pm 2.5 knots of accuracy, and in turn, this would be the highest accuracy available when using the developed image processing method. As the majority of ASIs use 5 knot increments this seems to be the most accuracy available in obtaining airspeed this way. While there is confidence in the developed method, the accuracy limit imposed by the ASI will always be present. While this results in a decrease 
in accuracy of the experimentally determined lift loads, it is believed that the developed method presented here would be sufficient enough to identify instances in which limit loads are exceeded.

\subsection{Future Work}

Although the results presented show that airspeed data can successfully be acquired through image processing and used to estimate aerodynamic loads of an aircraft in flight, there will always be room for improvement, refinement, and development. This section details areas of future consideration in the continuation of this project.

\subsubsection{Airspeed Acquisition via Image Processing}

While the developed image processing method of acquiring airspeed information produced very good results, there were a few instances where airspeed data was not attainable. Difficulties in object detection can arise due to occlusions of the object and scene, as well as changing appearance patterns of the object and the scene [38].

The first of such difficulties occurred when the view of the ASI was physically obstructed as shown in Figure 8.1(a). Detection of the ASI needle can be attained with a partial occlusion, but not complete occlusion of the object. This limitation imposed by complete obstruction was also experienced in work outlined in [39]. The only recommendation to avoid this issue is placement of the camera that has a low chance of allowing obstructions. Additionally, the changing appearance of the scene occurred when the aircraft was facing the direction of the sun and the resulting glare obscured the ASI as shown in Figure 8.1(b). Once again, this complete occlusion can not be processed.

The second difficulty occurred as a result of shadows cast on the ASI from the sunlight. As shown in Figure 8.1(c), a partial shadow on any portion of the ASI would not produce a usable binary image from which the airspeed could be determined. This partial occlusion of the scene has been shown to be dealt with using object tracking (as opposed to solely object detection) in [39]. The ability to track the position and size of an object, in this case 


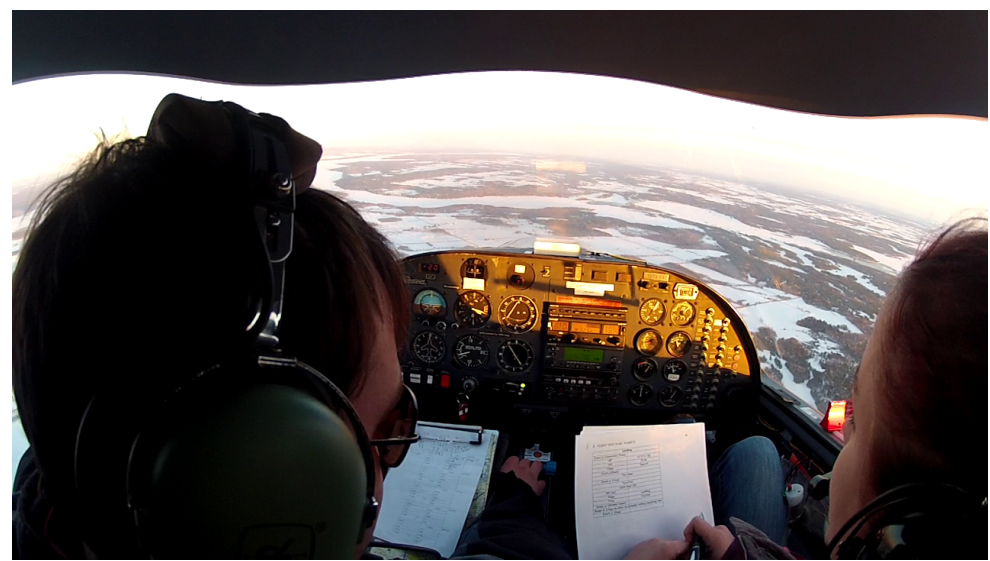

(a)

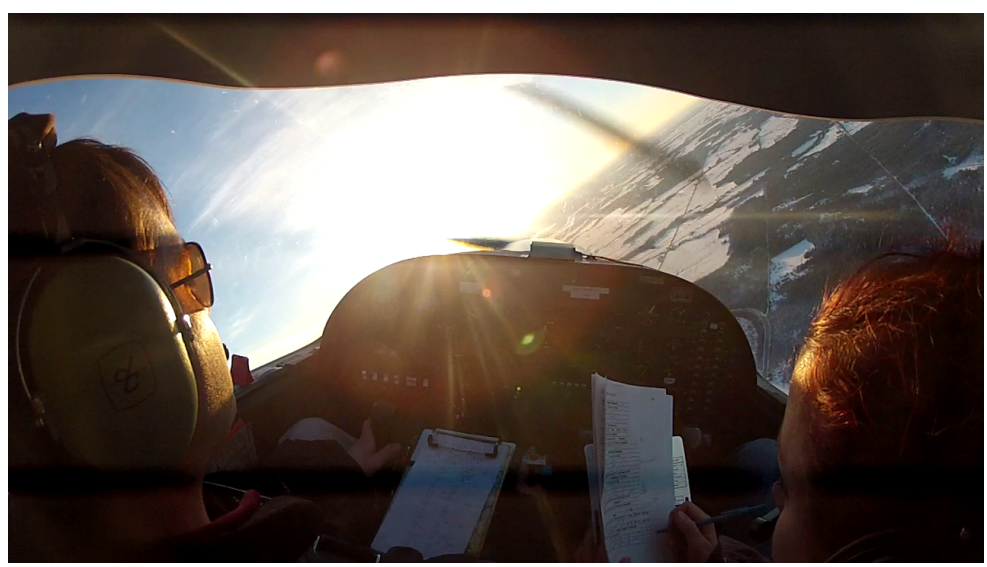

(b)

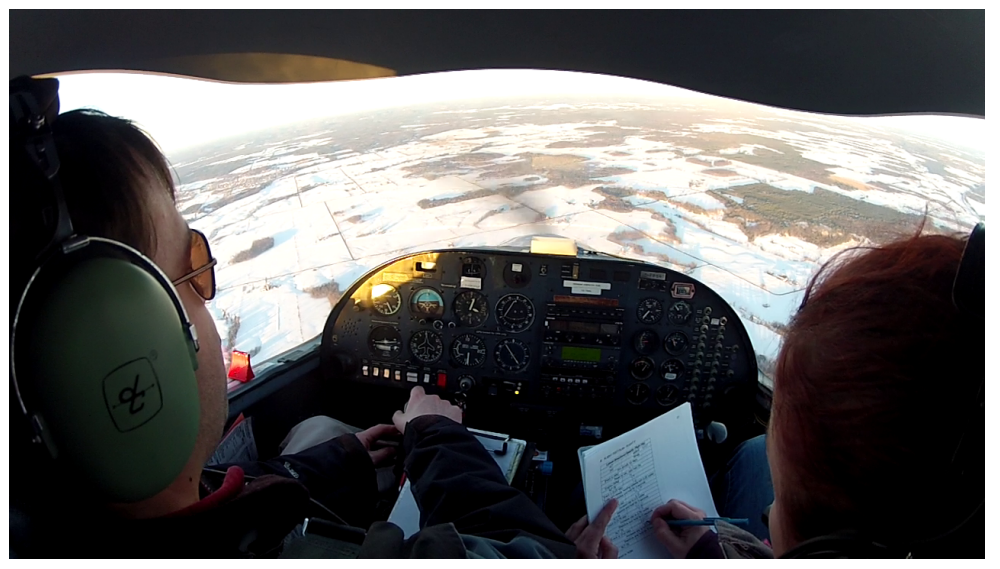

(c)

Figure 8.1: Examples of instances where airspeed data could not be acquired due to: (a) physical obstruction (b) sunlight glare (c) shadows cast on the ASI. 
the needle of the ASI, throughout a video sequence could potentially allow for a statistical algorithm to increase object detection accuracy [36]. The list of these algorithms are extensive, some of which can be found in [36] and [40].

Furthermore, an embedded system similar to those used in autonomous surveillance cameras could also be investigated. These systems are autonomous in an energy and memory point of view [40]. This autonomy allows them to operate over long periods of time and the energy and memory savings would be quite beneficial to a system installed on-board an aircraft.

The largest area of improvement is to increase the number of usable frames that can be used in the image processing method to acquire airspeed information. As discussed in Section 6.2.1 and shown in Figures 8.1(b) and 8.1(c), problems associated with image quality could be solved using a combination of polarizing lenses to decrease glare and decrease significant fluctuations in brightness (see Figure 6.3). Continuing with image quality, although a high-resolution camera was used in this method, only a $60 \times 60$ pixel region out of a potential $720 \times 640$ region was used. An improvement in the usable image region could improve the tracking of the ASI needle. Simply focusing and zooming the camera on the ASI would accomplish this, however, this could introduce camera vibrations. These vibrations were very low in the setup described here, and were not a concern during the image processing.

The use of image processing in sense-and-avoidance systems is presented in [41]. This work focuses on the development and flight performance analysis of an image-processing technique aimed at detecting flying obstacles in airborne images. Discussed in [41], are false alarms in the detection of flying objects due to sun glares captured in the image resulting in more than $15 \%$ of the pixels being saturated. To overcome these false alarms an algorithm was employed to evaluate the percentage of saturated pixels in the whole image and respond accordingly in implementing the object detection algorithm used for collision avoidance. A similar implementation can be applied to the developed airspeed acquisition presented here. However, due to the ASI needle being a much smaller object needing to be detected in comparison to another aircraft or other airborne obstacle as in [41], it is 
expected that this would entail a complex implementation of such an algorithm.

\subsubsection{Aerodynamic Load Determination and the Panel Method}

The panel method presented in Chapter 7 is representative of a three-dimensional wing in a two-dimensional flow, thus a number of flight segments cannot be analyzed. Examples of such segments include turns and side-slips, where the flow over the wings were not completely chord-wise and had significant span-wise flow. Implementing wing-tip wake panels, similar to those used at the trailing edge, a three-dimensional flow could be analyzed granted that the flow direction and magnitude over the aircraft wing in flight could be measured. Commonly, this is accomplished through the use of at least two pitot tubes [42], but from a purely certification viewpoint this seems quite complex but can be investigated.

The steady flow assumption used in Chapters 3 and 4, while sufficient for the majority of flight segments, can be expanded to include unsteadiness. Unsteady flow exists in important phenomena such as rapid manoeuvres, response to atmospheric turbulence, and flutter [43], but the time and effort in modeling this component may or may not be needed based on the flight regime of the particular aircraft being analyzed.

Furthermore, the simplicity of the panel method allows it to be expanded to include other sections of the aircraft such as the fuselage, empennage or any other structural elements. This would provide an increased understanding of the load history for other aircraft structures and could be used to analyze the interactions between the structures.

\subsection{Potential Applications}

The equipment used in this work, excluding the aircraft of course, amount to less than $\$ 500$ CAD. While this work was focused on aerodynamic load determination, the variety of the ArduPilot's on-board sensors could log many other aircraft parameters including: location, attitude, altitude, and heading/direction. The developed image processing method could also be expanded to obtain information from other cockpit flight instruments. This conceivably includes: engine speed, oil pressure, navigation equipment, the automatic direction 
finder, the course deviation indicator, and communication equipment. The range of information that could be obtained using an inexpensive ArduPilot/camera combination could be used to increase the safety in the small general aviation market segment. Being able to record flight data and reconstruct accidents would be a definite asset to any aviation safety organization. This ability could potentially also be a very useful teaching tool for the many students who partake in flight instructions. Being able to record flights then review, assess, and improve upon them while on the ground is a capability that is not easily accessible at the moment. 


\section{List of References}

[1] "Flight Schools." http://www.bestaviation.net/flightschool, 2015. Best Aviation.

[2] "Flying Clubs in Canada." http://flying-club.org/fc/fcoca.asp, 2015. My FBO.

[3] J. Quinlivan, "NASA-ACEE/Boeing 737 Graphite-Epoxy Horizontal Stabilizer Service," Technical Report N95-28489, NASA and Boeing Commercial Airplane Group, Seattle, Washington, 1992.

[4] CompositesWorld, Aerospace Composites: A Design and Manufacturing Guide. Wheat Ridge, CO: Gardner Publications Inc., 2008.

[5] G. Gardiner, "SAMPE Europe Highlights: Composites Face Challenges in Next Commercial Airframes." http://www.compositesworld.com/blog/post/sampe-europehighlights-composites-face-challenges-in-next-commercial-airframes, April 2014. Composites World.

[6] D. K. A. Baker, S. Dutton, ed., Composite Materials for Aircraft Structures. Reston, VA: American Institute of Aeronautics and Astronautics, 2nd ed., 2004.

[7] R. Kimberlin, Flight Testing of Fixed-Wing Aircraft. Reston, Virginia: American Institute of Aeronautics and Astronautics, 2003.

[8] E. Morelli, "Efficient Global Aerodynamic Modeling from Flight Data," Technical Memo 23681, NASA Langley Research Center, Hampton, Virginia, 2012.

[9] B. Stevens and F. Lewis, Aircraft Control and Simulation. New York, NY: John Wiley and Sons, 1992.

[10] T. Jordan, "Airborne Subscale Transport Aircraft Research Testbed - Aircraft Model Development," Technical Report 32785, NASA Langley Research Center, Hampton, Virginia, 1992.

[11] L. K. et al., "Operational Loads Monitoring of a Fleet of Beech 1900D Aircraft," Technical Paper 2008-01-2232, SAE International SP-2214, Wichita, Kansas, 2008. 
[12] D. R. Grossi, "Aviation Recorder Overview," Conference Proceeding International Symposium on Transportation Recorders, National Transportation Safety Board, Arlington, Virginia, May 3-5, 1999.

[13] D. Gilbert, "Cost Benefit Analysis of ICAO's Flight Data Recorder Recommendations," Technical Report 101047, Civil Aviation Safety Authority, Perth, Australia, September $21,2012$.

[14] "Reassessment of the Responses to Aviation Safety Recommendation A03-08 - Image (Video) Recording," Technical Report RecA0308, Transportation Safety Board of Canada, Gatineau, Canada, 2003.

[15] "Accident Investigation and Prevention Divisional Meeting," Technical Report AIG/08-WP/32, International Civil Aviation Organziation, Montreal, Canada, September 4, 2008.

[16] "Aviation Occcurences 2013 Statistical Summary," Technical Report SSEA-SSAO2013, Transportation Safety Board of Canada, Gatineau, Canada, 2014.

[17] "Diamond Aircraft Industries." http://www.diamondaircraft.com, April 2013. Diamond Aircraft Industries.

[18] "Useful Life and Inspections." http://whycirrus.com/engineering/useful-lifeinspections.aspx, December 2013. Cirrus Aircraft.

[19] "DA20 Flight Manual," Manual DA202-20AFM-20-18, Diamond Aircraft Industries, January 18, 2012.

[20] J. Katz and A. Plotkin, Low-Speed Aerodynamics. New York, NY: Cambridge University Press, 2001.

[21] F. M. White, Fluid Mechanics. Toronto, Canada: McGraw Hill, 5th ed., 2003.

[22] J. Moran, An Introduction to Theoretical and Computational Aerodynamics. Toronto, Canada: John Wiley and Sons, 1984.

[23] J. L. Hess and A. M. Smith, "Calculation of Potential Flow About Arbitrary Bodies," Technical Report Vol. 8, Progress in Aeronautical Sciences, 1967.

[24] J. L. Hess, "Calculation of Potential Flow About Arbitrary Three-Dimensional Lifting Bodies," Technical Report MDC-J5679-01, Department of the Navy Naval Air Systems Command, Long Beach, California, 1972.

[25] "Flight Training Manual," Manual TP1102E, Transport Canada, 2004.

[26] "Carleton University Katana Flight Test Plan," Manual ADL/13/JP/1, Applied Dynamics Laboratory, Ottawa, Canada, 2014. 
[27] "MPU-6000 and MPU-6050 Product Specification." http://www.invensense.com/ mems/gyro/documents/PS-MPU-6000A.pdf, May 2012. InvenSense Inc.

[28] "MS5611-01BA03 Barometric Pressure Sensor." http://www.measspec.com/downloads/MS5611-01BA03.pdf, October 2012. Measurement Specialties.

[29] “3-Axis Digital Compass IC HMC5883L." http://www51.honeywell.com/aero/common/ documents/myaerospacecatalog-documents/ Defense Brochuresdocuments/HMC5883L 3-AxisDigitalCompassIC.pdf, April 2010. Honeywell International Inc.

[30] "Mediatek - 3329 Datasheet." https://docs.google.com/file/d/0BdHj7E2weiiNmUzN DA3OTktNTNhNy00Y2Y5LTg0YTQtMzIyNzJhZmFiNjcy/edit?hl=en, April 2010. MediaTek.

[31] "MPXV7002 Series." http://www.freescale.com/files/sensors/doc/datasheet/ MPXV7002.pdf, May 2013. Freescale Semiconductor.

[32] "GoPro Hero 3." http://shop.gopro.com/cameras/hero3-white/CHDHE-302master.html, May 2013. GoPro Inc.

[33] "Canadian Aviation Regulations." http://laws-lois.justice.gc.ca/eng/regulations/SOR96-433/FullText.html, December 2013. Transport Canada.

[34] K. Saeed, S. H. Shaikh, and N. Chaki, Moving Object Detection Using Background Subtraction. London, United Kingdom: Springer, 2014.

[35] M. Adler, T. Boutell, J. Bowler, et al., "Portable Network Graphics (PNG) Specification," Manual ISO/IEC 15948:2003 (E), World Wide Web Consortium, 2003.

[36] B. Cyganek, Object Detection and Recognition in Digital Images: Theory and Practice. West Sussex, United Kingdom: John Wiley and Sons, 2013.

[37] W. J. McCroskey, "A Critical Assessment of Wind Tunnel Results for the NACA 0012 Airfoil," Technical Memo 100019, Aeroflightdynamics Directorate, U.S. Army Aviation Research and Technology Activity, Ames Research Center, Moffett Field, California, 1987.

[38] A. Yilmaz, O. Javed, and M. Shah, "Object Tracking: A Survey," in ACM Computing Surveys, vol. 38, 2006.

[39] T. Yang et al., "Real-time Multiple Objects Tracking with Occlusion Handling in Dynamic Scenes," Technical Report 710072, College of Automatic Control, Northwestern Polytechnical University, Xian, China, 2005.

[40] L. Wang, Machine Learning for Vision-Based Motion Analysis: Theory and Techniques. London, United Kingdom: Springer, 2011. 
[41] L. Forlenza, "Flight Performance Analysis of an Image Processing Algorithm for Integrated Sense-and-Avoid Systems," in International Journal of Aerospace Engineering, vol. 2012, 2012.

[42] G. Russo, Aerodynamic Measurements - From Physical Principles to Turnkey Instrumentation. Philadelphia, PA: Woodhead Publishing, 2011.

[43] H. Ashley, Aerodynamics of Wings and Bodies. New York: Addison-Wesley Publishing Company, 1965. 


\section{Appendix A}

W1 Transport
Canada $\begin{aligned} & \text { Transports } \\ & \text { Canada }\end{aligned} \quad$ FLIGHT AUTHORITY AUTORITÉ DE VOL

To. A : Ottawa Aviation Services

220 Hunt Club Rd. Ottawa, ON, K1V $1 \mathrm{C}$

\begin{tabular}{|c|c|c|}
\hline 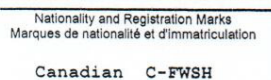 & 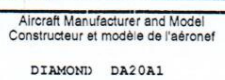 & 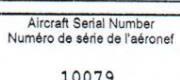 \\
\hline
\end{tabular}

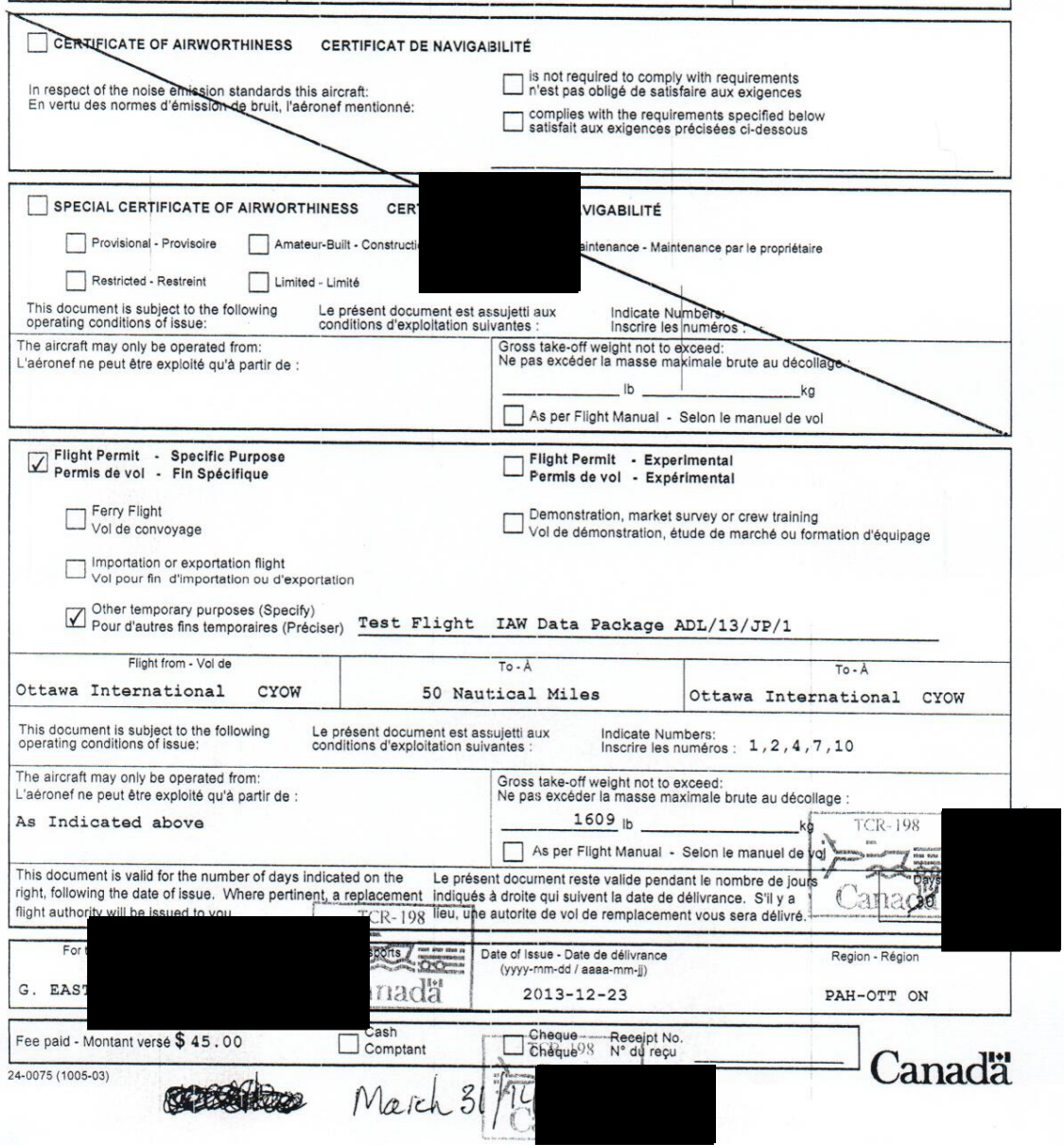


Operating Conditions

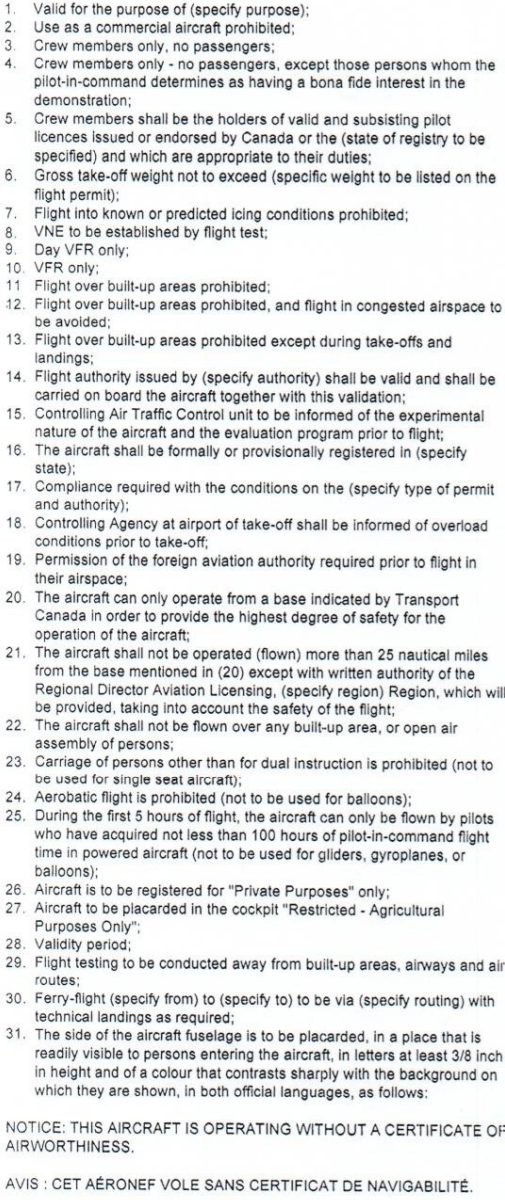

NOTICE: THIS AIRCRAFT IS OPERATING WITHOUT A CERTIFICATE OF

AVIS : CET AÉRONEF VOLE SANS CERTIFICAT DE NAVIGABILITE.

\section{Conditions d'exploitation}

Valide aux fins de (préciser les fins):

L'exploitation à titre d'aéronef commercial est interdite;

Membres d'équipage seulement - pas de passagers:

Membres dequipage seulement - pas de passagers, sauf les personnes qui de l'avis d

5. Les membres d'equipage doivent etre titulaires de licences de pllote valides et en vigueur délivrées ou annotées par le Canada ou (préciser |'Etat d'immatriculation) et correspondant à leurs fonctions.

6. Ne pas excéder la masse maximale brute au décollage (qui doit être indiquée sur le permis de vol);

7. Vol interdit dans des conditions de givrage existantes ou prévues:

La VNE doit etre etablie par essai en vo:

0. VFR seulement;

11. Le survol des zones bâties est interdit;

2. Le survol des zones báties est interdit, et le vol dans un espace aérien

3. Le survol des zones baties est interdit, sauf au décollage et à

l'atterrissage:

- L'autorité de vol dellivrée par (préciser l'autorité) doit être en vigueur et se trouver à bord de l'aéronef avec la présente validation

Atre in en controle de la circulation aérienne qui exerce le controle doit 政

programme devaluation:

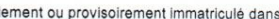

17. La conformite a

avec les conditions figurant sur le (préciser le type de 'organis autorite) est obligatoire;

informé a quile exerce le contrôle à l'aéroport de décollage doit être Le vol dans l'espace aérien étranger est interdit, sauf avec l'autorisation préalable de l'autorité de l'aviation civile thrang se en

. L'aéronef ne peut etre exploité qu'à partir de la base précisée par Transports Canada de façon à garantir le degré optimal de sécurite d'exploitation de l'aérones:

21. L'aéronét ne peut être exploité que dans une zone d'un rayon maximun de $25 \mathrm{NM}$ de la base mentonnes a lalinea 20 , sauf avec l'autorisation corite du directeur régional de la navigablilté, région (préciser la région) .llest interdit de survoler des zones bâties ou des . II est interdit de transporter des personnes sauf pour linstruction e double commande (ne pas utiliser dans le cas des aeronets

24. Le vol d'acrobatie aérienne est interdit (ne pas utiliser dans le cas de ballons):

25. Seul un pllote ayant accumule au moins 100 heures de vol à titre de commandant de bord d'aéronefs propulsés par un organe moteur est autorise piloter cet aeronef au cours des cinq premières heures de vol (ne pas ulliser dans le cas des planeurs, des autogires ou des ballon

27. Une affichette \& Restreint - fins agricoles seulement pouti Atre dans le poste de pilotage:

8. Période de validitié

29. Lessais en vol doivent être effectués hors des zones báties, de

2. Le vol de convoyage doites aeriennes;

(préciser la destination) via (préciser la (preciser la partance) à

31. Une affichette doit être apposée au coté du fuselage de l'aèronef, en un endroit facllement visible pour les personnes qui montent dans l'aérone en lettres d'au moins $3 / 8$ pouce de hauteur et d'une couleur contrastant clairement avec le fond sur lequel elles sont apposées, dans les deux

AVIS : CET AERONEF VOLE SANS CERTIFICAT DE NAVIGABILITÉ NOTICE: THIS AIRCRAFT IS OPERATING WITHOUT A CERTIFICATE OF 


\section{Appendix B}

\section{Sun Presence Detector}
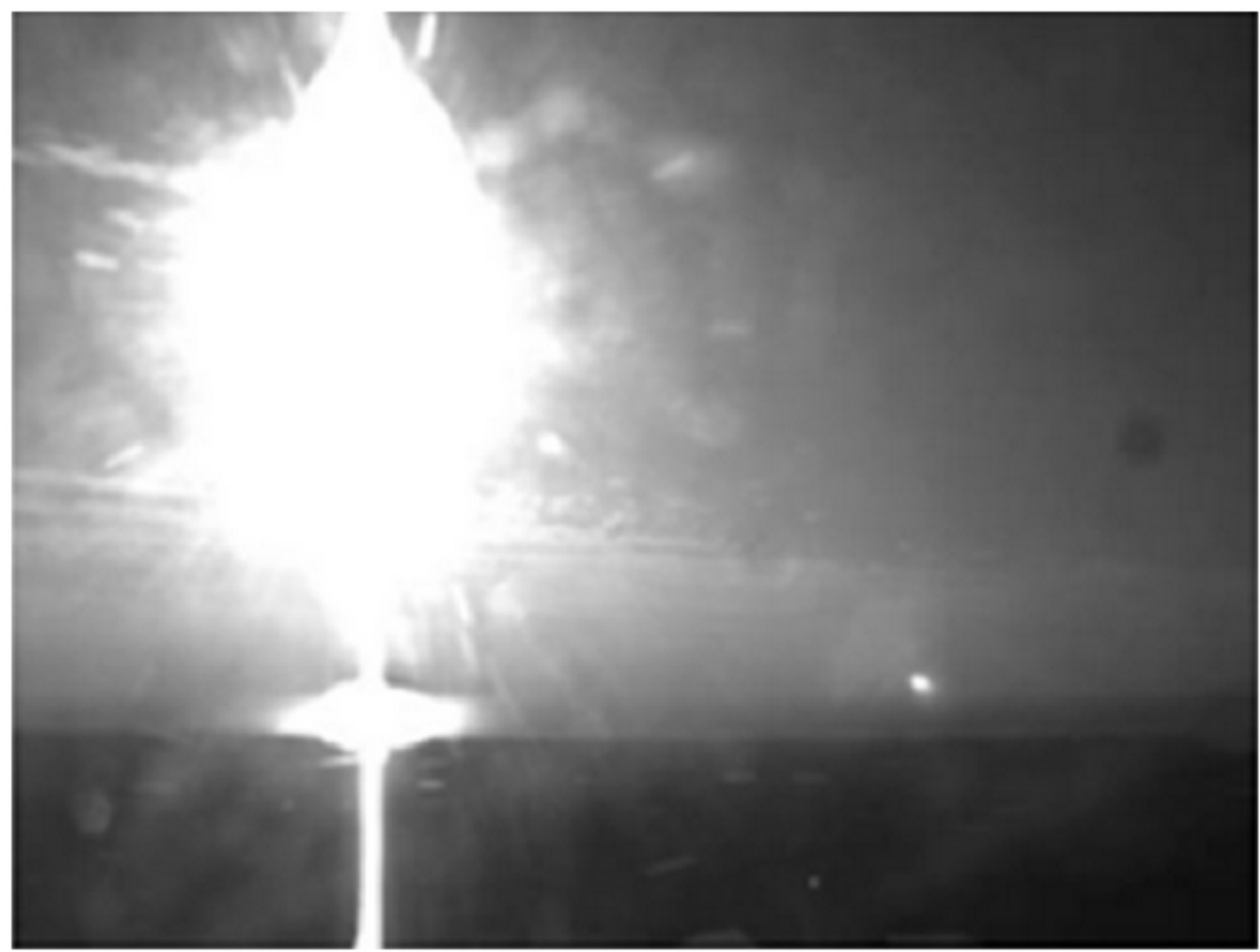

Figure B.1: An example of the sun presence detected by a "Sun Detector" algorithm used in [41]. 